\title{
An Introduction to Rough Paths
}

\author{
Antoine Lejay ${ }^{1}$ — Projet Tosca (INRIA / IECN)
}

Abstract: This article aims to be an introduction to the theory of rough paths, in which integrals of differential forms against irregular paths and differential equations controlled by irregular paths are defined. This theory makes use of an extension of the notion of iterated integrals of the paths, whose algebraic properties appear to be fundamental. This theory is well-suited for stochastic processes.

Keywords: controlled differential equations, integration against irregular paths, $p$-variation, stochastic processes, iterated integrals, Chen series, geometric multiplicative functional

AMS Classification: Primary 60H10, 34A10; Secondary 34F05

Published in Séminaire de probabilités XXXVII. vol. 1832 of Lecture Notes in Mathematics, pp. 1-59. Springer-Verlag, 2003

Archives, links \& reviews:

○ MR number: 2053040

○ HAL: inria-00102184

○ DOI: $10.1007 / \mathrm{b} 94376$

${ }^{1}$ Current address: Projet TOSCA (INRIA / IECN ), IECN, Campus scientifique, BP 239, 54506 Vandœuvre-lès-Nancy CEDEX, France

E-mail: Antoine.Lejay@iecn.u-nancy.fr 


\section{Introduction}

This article is an introduction to the theory of rough paths, which has been developed by T. Lyons and his co-authors since the early '90s. The main results presented here are borrowed from [32, 36]. This theory concerns differential equations controlled by irregular paths and integration of differential forms against irregular trajectories. Here, $x$ is a continuous function from $[0,1]$ to $\mathbb{R}^{d}$, and the notion of irregularity we use is that of $p$-variation, as defined by $\mathrm{N}$. Wiener. This means that for some $p \geqslant 1$,

$$
\sup _{\substack{k \geqslant 1,0 \leqslant t_{0} \leqslant \ldots \leqslant t_{k} \leqslant 1 \\ \text { partition of }[0,1]}} \sum_{i=0}^{k-1}\left|x_{t_{i+1}}-x_{t_{i}}\right|^{p}<+\infty .
$$

As we will see, the integer $\lfloor p\rfloor$ plays an important role in this theory.

In probability theory, most stochastic processes are not of finite variation, but are of finite $p$-variation for some $p>2$. We show in Sect. 10 how to apply this theory to Brownian motion. But the theory of rough paths could be used for many types of processes, as presented in Sect. 12.

Firstly, we give a meaning to the integral

$$
\int_{0}^{t} f\left(x_{s}\right) \mathrm{d} x_{s}, \text { or equivalently, } \int_{x([0, t])} f
$$

for a differential form

$$
f(x)=\sum_{i=1}^{d} f_{i}(x) \mathrm{d} x^{i} .
$$

We are also interested in solving the controlled differential equation

$$
\mathrm{d} y_{t}=f\left(y_{t}\right) \mathrm{d} x_{t},
$$

where $f$ is the vector field

$$
f(y)=\sum_{i=1}^{d} f_{i}(y) \frac{\partial}{\partial x_{i}} .
$$

This will be done using Picard's iteration principle, from the result on integration of one-forms. Using the terminology of controlled differential equations, $x$ is called a control.

The theory of rough paths also provided some results on the continuity of the map $x \mapsto y$, where $y$ is given either by (1.1) or (1.3).

The theory of rough paths may be seen as a combination of two families of results: 
(1) Integration of functions of finite $q$-variation against functions of finite $p$ variation with $1 / p+1 / q>1$ as defined by L.C. Young in [52].

(2) Representation of the solutions of (1.3) using iterated integrals of $x$ : this approach is in fact an algebraic one, much more than an analytical one.

Let us give a short review of these notions.

\section{(1) Young's integral}

Let $x$ and $y$ be two continuous functions respectively $1 / p$ and $1 / q$-Hölder continuous with $\theta=1 / p+1 / q>1$. Then, Young's integral $\int_{s}^{t} y_{r} \mathrm{~d} x_{r}$ of $y$ against $x$ is defined as the limit of $I_{s, t}(\Pi)=\sum_{i=0}^{k-1} y_{t_{i}}\left(x_{t_{i+1}}-x_{t_{i}}\right)$ when the mesh of the partition $\Pi=\left\{t_{i} \mid s \leqslant t_{0} \leqslant \cdots \leqslant t_{k} \leqslant t\right\}$ of $[s, t]$ goes to zero (see for example [12, [52]). It is possible to choose a point $t_{j}$ in $\Pi$ such that

$$
\left|I_{s, t}(\Pi)-I_{s, t}\left(\Pi \backslash\left\{t_{j}\right\}\right)\right| \leqslant \frac{1}{(\operatorname{Card} \Pi)^{\theta}} C|t-s|^{\theta}
$$

for some constant $C$ that depends only on the Hölder norm of $x$ and $y$. Whatever the size of the partition $\Pi$ is, $\left|I_{s, t}(\Pi)\right| \leqslant\left|y_{s}\left(x_{t}-x_{s}\right)\right|+|t-s|^{\theta} \zeta(\theta)$, where $\zeta(\theta)=$ $\sum_{n \geqslant 1} 1 / n^{\theta}$. The limit of $I_{s, t}(\Pi)$ as the mesh of $\Pi$ goes to 0 may be considered.

One may be tempted to replace $y$ by $f(x)$, where the regularity of $f$ depends on the irregularity of $x$. But to apply directly the proof of L.C. Young, one has to assume that $f$ is $\alpha$-Hölder continuous with $\alpha>p-1$, which is too restrictive as soon as $p \geqslant 2$. To bypass this limitation, we construct when $x_{t} \in \mathbb{R}^{d}$ the integral $\sum_{j=1}^{d} \int_{s}^{t} f_{j}\left(x_{r}\right) \mathrm{d} x_{r}^{j}$ as

$$
\begin{aligned}
\lim _{\operatorname{mesh}(\Pi) \rightarrow 0} \sum_{i=0}^{k-1}\left(\sum_{j=1}^{d} f_{j}\left(x_{t_{i}}\right)\left(x_{t_{i+1}}^{j}-x_{t_{i}}^{j}\right)+\sum_{j_{1}, j_{2}=1}^{d} \frac{\partial f_{j_{1}}}{\partial x_{j_{2}}}\left(x_{t_{i}}\right) \mathbf{x}_{t_{i}, t_{i+1}}^{i,\left(j_{2}, j_{1}\right)}\right. \\
\left.+\cdots+\sum_{j_{1}, \ldots, j_{\lfloor p\rfloor}=1}^{d} \frac{\partial^{\lfloor p\rfloor-1} f_{j_{1}}}{\partial x_{j_{\lfloor p\rfloor}} \cdots \partial x_{j_{2}}}\left(x_{t_{i}}\right) \mathbf{x}_{t_{i}, t_{i+1}}^{\lfloor p\rfloor,\left(j_{\lfloor p\rfloor}, \ldots, j_{1}\right)}\right) \\
+\cdots
\end{aligned}
$$

with formally

$$
\mathbf{x}_{s, t}^{i,\left(j_{i}, \ldots, j_{1}\right)}=\int_{s \leqslant s_{i} \leqslant \cdots \leqslant s_{1} \leqslant t} \mathrm{~d} x_{s_{i}}^{j_{i}} \cdots \mathrm{d} x_{s_{1}}^{j_{1}} .
$$

This expression (1.4) is provided by the Taylor formula on $f$ and the more $x$ is irregular, i.e., the larger $p$ is, the more regular $f$ needs.

What makes the previous definition formal is that the "iterated integrals" of $x$ have to be defined, and there is no general procedure to construct them, nor are they unique. The terms $\mathbf{x}^{k,\left(i_{1}, \ldots, i_{k}\right)}$ for $k=2, \ldots,\lfloor p\rfloor$ are limits of iterated integrals 
of piecewise smooth approximations of $x$, but they are sensitive to the way the path $x$ is approximated. Due to this property, the general principle in the theory of rough paths is:

The integral $\sum_{j=1}^{d} \int_{s}^{t} f_{j}\left(x_{r}\right) \mathrm{d} x_{r}^{j}$ is not driven by $x$ but, if it exists, by $\mathbf{x}=\left(\mathbf{x}^{1,\left(i_{1}\right)}, \mathbf{x}^{2,\left(i_{1}, i_{2}\right)}, \ldots, \mathbf{x}^{\lfloor p\rfloor,\left(i_{1}, \ldots, i_{\lfloor p\rfloor}\right)}\right)_{i_{1}, \ldots, i_{\lfloor p\rfloor}=1, \ldots, d}$ corresponding formally to (1.5).

\section{(2) Formal solutions of differential equations}

Assume now that $x$ is smooth, and let $\mathbf{x}_{s, t}^{k,\left(i_{1}, \ldots, i_{k}\right)}$ be its iterated integrals defined by (1.5). Given some indeterminates $X^{1}, \ldots, X^{d}$, we consider the formal noncommutative power series:

$$
\Phi([s, t], x)=1+\sum_{k \geqslant 1} \sum_{\left(i_{1}, \ldots, i_{k}\right) \in\{1, \ldots, d\}^{k}} X^{i_{1}} \cdots X^{i_{k}} \mathbf{x}_{s, t}^{k,\left(i_{1}, \ldots, i_{k}\right)} .
$$

As first proved by K.T. Chen in [6], $\Phi([s, t], x)$ fully characterizes the path $x$, and for all $s \leqslant u \leqslant t$,

$$
\Phi([s, u], x) \Phi([u, t], x)=\Phi([s, t], x) .
$$

This relation between iterated integrals is also used to prove that the limit in (1.4) exists. If exp is the non-commutative exponential (defined by a power series), then there exists a formal series $\Psi([s, t], x)$ such that $\Phi([s, t], x)=\exp (\Psi([s, t], x))$ and

$$
\Psi([s, t], x)=\sum_{k \geqslant 1} \sum_{\left(i_{1}, \ldots, i_{k}\right) \in\{1, \ldots, d\}^{k}} F_{\left(i_{1}, \ldots, i_{d}\right)}\left(X^{1}, \ldots, X^{d}\right) \mathbf{x}_{s, t}^{k,\left(i_{1}, \ldots, i_{k}\right)}
$$

where $F_{\left(i_{1}, \ldots, i_{d}\right)}\left(X^{1}, \ldots, X^{d}\right)$ belongs to the Lie algebra generated by the indeterminates $X^{1}, \ldots, X^{d}$, i.e., the smallest submodule containing $X^{1}, \ldots, X^{d}$ and closed under the Lie brackets $[Y, Z]=Y Z-Z Y$.

If $f=\left(f_{1}, \ldots, f_{d}\right)$ and each of the $f_{i}$ is linear, i.e., $f_{i}(y)=C_{i} y$ where $C_{i}$ is a matrix, then the solution $y$ of (1.3) is equal to

$$
y_{t}=\exp (\widehat{\Psi}([s, t], x)) y_{s}
$$

where $\widehat{\Psi}([s, t], x)$ is equal to $\Psi([s, t], x)$ in which $X^{i}$ was replaced by the matrix $C_{i}$. If $f$ is not linear, but is for example a left-invariant vector field on a Lie group, then a similar relation holds, where $X^{i}$ is replaced by $f_{i}$, and the Lie brackets $[\cdot, \cdot]$ are replaced by the Lie bracket between vector fields. Here, the exponential is replaced by the map defining a left-invariant vector field from a vector in the Lie algebra, i.e., the tangent space at 0 (see for example [13]). 
This result suggests that when one knows $x$, he can compute its iterated integrals and then formally solve (1.3) by replacing the indeterminates by $f$. In fact, when $x$ is irregular, the solution $y$ of (2.2) will be constructed using Picard's iteration principle, i.e., as the limit of the sequence $y^{n}$ defined by $y_{t}^{n+1}=$ $y_{0}+\int_{0}^{t} f\left(y_{r}^{n}\right) \mathrm{d} x_{r}$. But it corresponds, if $\left(x^{\delta}\right)_{\delta>0}$ is a family of piecewise smooth approximations of $x$ and $f$ is smooth, to

$$
y=\lim _{\delta \rightarrow 0} y^{\delta} \text { with } y_{t}^{\delta}=\exp \left(\widehat{\Psi}\left([0, t], x^{\delta}\right)\right) y_{0} .
$$

However, in the previous expression, we need all the iterated integrals of $x$. Yet, even if $x$ is irregular, there exists a general procedure to compute them all, assuming we know $\mathbf{x}$ defined formally by (1.5). However, different families of approximations $\left(x^{\delta}\right)_{\delta>0}$ may give rise to different $\mathbf{x}$. Thus, the solution $y$ of (1.3) given by the theory of rough paths depends also on $\mathbf{x}$ and not only on $x$, and the general principle stated above is also respected.

\section{Geometric multiplicative functionals}

As we have seen, we need to construct an object $\mathbf{x}$ corresponding to the iterated integrals of an irregular path up to a given order $\lfloor p\rfloor$. Since $\mathbf{x}$ may be reached as the limit of smooth paths together with its iterated integrals, $\mathbf{x}$ may be seen as an extension by continuity of the function $x \mapsto \Phi([s, t], x)$ giving the truncated Chen series, where $X^{i_{1}} \cdots X^{i_{k}}$ is set to 0 as soon as $k>\lfloor p\rfloor$. This means in particular that, at the limit, we keep the algebraic relation (1.6). This means that $\mathbf{x}_{s, t}$ may be seen as a formal non-commutative polynomial (in the text, we use tensor products, but this is equivalent in the finite-dimensional setting). Set $\mathbf{x}_{s, t}^{0}=1$ and $\mathbf{x}_{s, t}^{k}=\sum_{\left(i_{1}, \ldots, i_{k}\right)} X^{i_{1}} \cdots X^{i_{k}} \mathbf{x}^{k,\left(i_{1}, \ldots, i_{k}\right)}$. Thus, $\mathbf{x}_{s, t}=1+\mathbf{x}_{s, t}^{1}+\cdots+\mathbf{x}_{s, t}^{\lfloor p\rfloor}$. The relation (1.6) becomes

$$
\mathbf{x}_{s, t}=\mathbf{x}_{s, u} \mathbf{x}_{u, t} \text { for all } 0 \leq s \leq u \leq t \leq 1
$$

This means that, if $\mathbf{x}$ lies above $x$,

$$
\begin{aligned}
\mathbf{x}_{s, t}^{1} & =\mathbf{x}_{s, u}^{1}+\mathbf{x}_{u, t}^{1} \text { with } \mathbf{x}_{s, t}^{1, i}=x_{t}^{i}-x_{s}^{i} \\
\mathbf{x}_{s, t}^{2} & =\mathbf{x}_{s, u}^{2}+\mathbf{x}_{u, t}^{2}+\mathbf{x}_{s, u}^{1} \mathbf{x}_{u, t}^{1}, \\
\vdots & =\vdots+\vdots+\cdots
\end{aligned}
$$

Thus for $k=1, \ldots,\lfloor p\rfloor$, one can compute $\mathbf{x}_{s, t}^{k}$ from $\mathbf{x}_{s, u}^{i}$ and $\mathbf{x}_{u, t}^{i}$ when these quantities are known for $i=1, \ldots, k$.

The objects $\mathbf{x}$ that could be reached as an extension of the truncated Chen series $\Phi([s, t], x)$ and satisfying (1.7) are called geometric multiplicative functionals. 
Our goal is to construct from $\mathbf{x}$ new geometric multiplicative functionals $\mathbf{z}$. For example, the integral $\int f\left(x_{r}\right) \mathrm{d} \mathbf{x}_{r}$ will itself be constructed as a geometric multiplicative functional. Remark that for $\mathbf{z}_{s, t}=\int_{s}^{t} f\left(x_{r}\right) \mathrm{d} \mathbf{x}_{r}$, (1.8) is no more than the Chasles relation.

The machinery we use to construct $\mathbf{z}$ is the following: We construct first an approximation $\mathbf{y}$ of $\mathbf{z}$. For example, if $x$ is of finite $p$-variation with $p<2$, we define $\mathbf{y}_{s, t}^{1}$ by $f\left(x_{s}\right)\left(x_{t}-x_{s}\right)$, which is an approximation of $\int_{s}^{t} f\left(x_{r}\right) \mathrm{d} x_{r}$.

The object $\mathbf{y}$ is a non-commutative polynomial, but $\mathbf{y}$ does not satisfy relation (1.7) in general. Thus, if $\Pi=\left\{t_{i} \mid 0 \leq t_{1} \leq \cdots \leq t_{k} \leq 1\right\}$ is a partition of $[0,1]$, we set

$$
\mathbf{y}_{s, t}^{\Pi}=\mathbf{y}_{s, t_{i}} \mathbf{y}_{t_{i}, t_{i+1}} \cdots \mathbf{y}_{t_{j-1}, t_{j}} \mathbf{y}_{t_{j}, t} .
$$

where $i$ and $j$ are the smallest and the largest integers such that $\left[t_{i}, t_{j}\right] \subset[s, t]$. At the first level of path, this relation reads

$$
\mathbf{y}_{s, t}^{\Pi, 1}=\sum_{i=1}^{d} X^{i}\left(\mathbf{y}_{s, t_{i}}^{1,(i)}+\mathbf{y}_{t_{i}, t_{i+1}}^{1,(i)}+\cdots+\mathbf{y}_{t_{j-1}, t_{j}}^{1,(i)}+\mathbf{y}_{t_{j}, t}^{1,(i)}\right) .
$$

But for $\mathbf{y}^{\Pi, 2}$, this relation implies all the $\mathbf{y}_{t_{j}, t_{j+1}}^{\Pi,}$ 's an $\mathbf{y}_{t_{j}, t_{j+1}}^{\Pi, 1}$ 's for $t_{j} \in \Pi$.

Of course, $\mathbf{y}^{\Pi}$ also fails to satisfy (1.7), except if $s, u$ and $t$ belong to $\Pi$. But provided one has a relation of the type

$$
\left|\mathbf{y}_{s, t}-\mathbf{y}_{s, u} \mathbf{y}_{u, t}\right| \leq \varepsilon(s, u, t)
$$

for a nice $\varepsilon$, then one could consider the difference between $\mathbf{y}_{s, t}^{\Pi}$ and $\mathbf{y}_{s, t}^{\Pi \backslash\left\{t_{j}\right\}}$ for an element $t_{j}$ in $\Pi \cap(s, t)$. If $t_{j}$ is well chosen, and the choice of $t_{j}$ is similar to the one done for the construction of Young's integral (see above), one could show that $\left|\mathbf{y}_{s, t}^{\Pi}\right| \leq C(s, t)$, for a function $C(s, t)$ that does not depend on the partition $\Pi$. One could then pass to the limit as the mesh of the partition $\Pi$ decreases to 0 . Of course, it has to be proved that under reasonable conditions, the limit, which we denote by $\mathbf{z}$, is unique and is a geometric multiplicative functional. Moreover, the work has to be done iteratively at each level of iterated integrals. Thus, if $\mathbf{z}^{1}, \ldots, \mathbf{z}^{k}$ are already constructed, one gains the fact that $\left(\mathbf{z}^{1}, \ldots, \mathbf{z}^{k}\right)$ satisfies (1.7), and $\mathbf{z}^{k+1}$ is constructed using the previous machinery.

\section{Defining the iterated integrals}

Since the previoulsy described procedure is general, anybody interested only in applying this theory could adopt the following point of view:

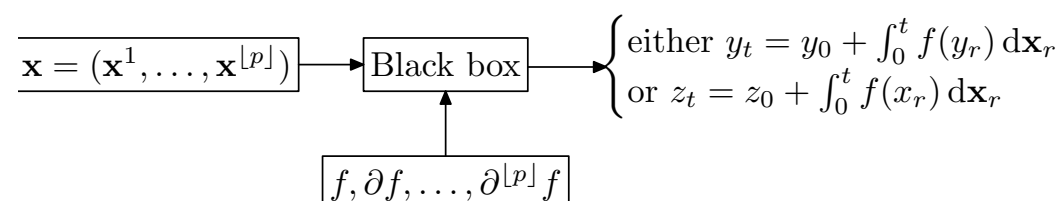


and focus on $\mathbf{x}$. In the previous section, we have seen how to construct new geometric multiplicative functionals from $\mathbf{x}$, but we have not said how $\mathbf{x}$ is constructed. We have already said that $\mathbf{x}$ may be difficult to construct. The most natural approach is to choose a piecewise smooth approximation $x^{\delta}$ of $x$ and to define $\mathbf{x}_{s, t}^{k,\left(i_{1}, \ldots, i_{k}\right)}$ as the limit of

$$
\int_{s \leqslant s_{k} \leqslant \cdots \leqslant s_{1} \leqslant t} \mathrm{~d} x_{s_{k}}^{i_{k}, \delta} \cdots \mathrm{d} x_{s_{1}}^{i_{1}, \delta} .
$$

For example, consider a $d$-dimensional Brownian motion $B$. As its trajectories are $\alpha$-Hölder continuous for any $\alpha<1 / 2$, they are of finite $p$-variation for any $p>2$. Hence, applying the theory of rough paths requires knowing the equivalent of the second-order iterated integrals of the Brownian motion. Let $B^{\delta}(\omega)$ be an approximation of $B(\omega)$. One knows that the convergence of $I_{s, t}^{\delta}(\omega)=\int_{s \leqslant s_{2} \leqslant s_{1} \leqslant t} \mathrm{~d} B_{s_{2}}^{i, \delta}(\omega) \mathrm{d} B_{s_{1}}^{j, \delta}(\omega)$ depends on the choice of $\left(B^{\delta}\right)_{\delta>0}$ (see [19, Sect. VI,-7, p. 392]). Besides, if for example, $B^{\delta}(\omega)$ is a piecewise linear approximation of $B(\omega)$ sampled along deterministic partitions, then the limit of $I_{s, t}^{\delta}$ is the Stratonovich integral $I_{s, t}=\int_{s \leqslant s_{2} \leqslant s_{1} \leqslant t} \circ \mathrm{d} B_{s_{2}}^{i} \circ \mathrm{d} B_{s_{1}}^{j}$ and is defined only as a limit in probability (Yet some recent works prove that for dyadic partitions, the convergence may be almost sure. See for example [24]). Another difficulty when we want to use rough paths is that we need to prove that $B^{\delta}$ and $I^{\delta}$ converge to $B$ and $I$ in the topology generated by the distance in $p$-variation, which is more complicated to use than the uniform norm.

Trajectories of stochastic processes represent a natural class of irregular paths for which one may require some integration theory. Thus, the theory of rough paths provides a pathwise theory of integration, but pathwise with respect to $\mathbf{x}$, and not to the stochastic process $x$. So, the difficulty is to construct the iterated integrals of the trajectories of $x$. However, it has to be noted that constructing $\mathbf{x}$ may be simpler than constructing stochastic integrals driven by $x$. For example, for the Brownian motion, $\int_{s \leqslant s_{2} \leqslant s_{1} \leqslant t} \circ \mathrm{d} B_{s_{2}}^{i} \circ \mathrm{d} B_{s_{1}}^{j}=A_{s, t}\left(B^{i}, B^{j}\right)+\frac{1}{2}\left(B_{t}^{i}-B_{s}^{i}\right)\left(B_{t}^{j}-B_{s}^{j}\right)$, where $A_{s, t}\left(B^{i}, B^{j}\right)$ is the Lévy area of $\left(B^{i}, B^{j}\right)$. This functional $A_{s, t}\left(B^{i}, B^{j}\right)$, which represents the area enclosed between the curve of $r \in[s, t] \mapsto\left(B_{r}^{i}, B_{r}^{j}\right)$ and its chord, was constructed by Paul Lévy (see for example [29]) before the construction of Itô or Stratonovich stochastic integrals.

Using the theory of rough paths, one has then to focus on the construction of $\mathbf{x}$ for given trajectories of $x$. This has some advantages, among which: (i) It is easier to define an object like $\int_{s}^{t}$ od $x_{r} \circ \mathrm{d} x_{r}$ for a stochastic process than $\int_{s}^{t} f\left(x_{r}\right) \circ \mathrm{d} x_{r}$ or solving $\mathrm{d} y_{t}=f\left(y_{t}\right) \circ \mathrm{d} x_{t}$. Section 12 contains a list of types of stochastic processes for which the theory of rough paths may be used, and then may be directly applied to solve differential equations. Moreover, the separation of $\mathbf{x}$ and $f$ may be advantageous since we also gain knowledge of the algebraic structure of $\mathbf{x}$ : see [37, 47] for an original application to Monte Carlo methods. (ii) A support theorem is imme- 
diate once we have one on $\mathbf{x}$ (see [24] for an application). (iii) Different piecewise smooth approximations of a stochastic process lead to different stochastic integrals. This is well known for Brownian motion, but generalizes immediately to different processes for which the theory of rough paths may be applied. Besides, this theory provides some explanations on the form of the corrective drift (see Sects. 6.2 and 10.2). (iv) Once $\mathbf{x}$ has been defined on a probability space $(\Omega, \mathcal{F}, \mathbb{P})$, then all the differential equations $\mathrm{d} y_{t}=f\left(y_{t}\right) \mathrm{d} x_{t}$ and the integrals $\int f\left(x_{t}\right) \mathrm{d} x_{t}$ are defined on the same set $\Omega_{0} \subset \Omega$ of full measure, whatever the function $f$ is.

In this article, we assume that the path $x$ takes its values in $\mathrm{V}=\mathbb{R}^{d}$, and that the differential forms or vector fields $f$ take their values in $\mathrm{W}=\mathbb{R}^{m}$. However, $\mathrm{V}$ and $\mathrm{W}$ could in fact be any Banach space, even of infinite dimension.

\section{Motivations}

This article does not give a full treatment of the theory of rough paths. But its aim is to give the reader sufficient information about this theory to help him to have a general view of it, and maybe to apply it. The reader who is interested in this theory can read either [32] or [36] to go further.

The theory of rough paths is suitable for trajectories of stochastic processes, since there are many types of stochastic processes for which it is possible to construct their "iterated integrals". Yet each application to a particular type of probabilistic problem may require a specific approach. As randomness plays no role in this theory, probability theory takes only a small place in this article. The reader is refered to Sect. 12 and to the bibliography for applications to stochastic analysis.

\section{Outline of the article}

For the sake of clarity, we explain in Sect. 2 how to integrate a differential form along a path of finite $p$-variation with $p \in[1,2)$, and then how to solve a differential equation controlled by such a path. In Sect. 3, we deal with paths of finite $p$ variation with $p \in[2,3)$. This is the most common case a probabilist could use. Besides, we think that understanding the situation in this case together with the proofs of Sect. 2 allows us to fully understand the general theory.

Sections 4 and 5 are devoted to introducing the basic algebraic results on iterated integrals. Section 6 gives the general definition of geometric multiplicative functionals, i.e., the objects $\mathbf{x}$ previously introduced, and some convergence results on them. The notion of almost multiplicative functional, which is the basic element to define an integral, is presented in Sect. 7. The general results on integration of one-forms and controlled differential equations are given in Sects. 8 and 9 without proof. 
A practical example is presented in Sects. 10 and 11, where the theory is applied to the Brownian motion. Section 11 also contains a method to compute the $p$ variation of a multiplicative functional.

Finally, Sect. 12 contains a list, which attempts to be as complete as possible at the date of writing, of bibliographic references on works using the theory of rough paths. This article ends with some bibliographical and historical notes.

Note. At the first reading, the reader may go directly from the end of Sect. 3 to Sect. 10 for an application to the Brownian motion.

\section{Acknowledgement}

I am glad to have been granted a post-doctoral position by the European Union's TMR Stochastic Analysis Network and to have benefited from Prof. T. Lyons' kind hospitality at Oxford University during the academic year 2000-2001. I have learned there, among other things, the basis of the theory of rough paths. I wish also thank all of those, and especially L. Coutin, M. Émery, M. Ledoux and N. Victoir, who have read the early versions of the manuscript, pointed out some mistakes, and made valuable comments.

\section{The case of a not so irregular control}

Set $\Delta^{+}=\left\{(s, t) \in[0,1]^{2} \mid 0 \leqslant s \leqslant t \leqslant 1\right\}$. Through all this article, we use a function $\omega$ from $\Delta^{+}$to $\mathbb{R}_{+}$satisfying the following assumption.

Assumption 1. The function $\omega: \Delta^{+} \rightarrow \mathbb{R}_{+}$is such that

(i) The function $\omega$ is bounded.

(ii) The function $\omega$ is continuous near the diagonal, i.e., on $\{(s, s) \mid s \in[0,1]\}$, and $\omega(s, s)=0$ for all $s \in[0,1]$.

(iii) For all $0 \leqslant s \leqslant u \leqslant t \leqslant 1$,

$$
\omega(s, u)+\omega(u, t) \leqslant \omega(s, t) .
$$

If follows immediately that for all $\theta \geqslant 1, \omega^{\theta}$ is also super-additive: $\omega(s, u)^{\theta}+$ $\omega(u, t)^{\theta} \leqslant \omega(s, t)^{\theta}$ for all $s \leqslant u \leqslant t \leqslant 1$.

Moreover, it is easily seen that for all $\varepsilon$, there exists some $\eta$ small enough such that $|t-s|<\eta$ implies that $\omega(s, t)<\varepsilon$ for all $(s, t) \in \Delta^{+}$. 


\subsection{Integration of a differential form along an irregular path}

In this section, we show that, provided one controls the value of $\left|x_{t}-x_{s}\right|^{p}$ for $p \in(1,2)$, then $\int_{0}^{t} f\left(x_{s}\right) \mathrm{d} x_{s}$ may be defined with Riemann sums.

Assumption 2. There exists a real $1 \leqslant p<2$ such that

$$
\left|x_{t}-x_{s}\right|^{p} \leqslant \omega(s, t) \text { for all }(s, t) \in \Delta^{+}
$$

for a function $\omega$ satisfying Assumption 1, For example, this is true if $x$ is $1 / p$ Hölder continuous, in which case, $\omega(s, t)=C|t-s|$ for some constant $C$.

The differential form $f$ defined by (1.2) is identified with $\left(f_{1}, \ldots, f_{d}\right): \mathbb{R}^{d} \mapsto$ $\left(\mathbb{R}^{m}\right)^{d}$. The function $f$ is bounded and $\alpha$-Hölder continuous, with $\alpha>p-1$.

Note that (2.2) together with (2.1) exactly means that $x$ is of finite $p$-variation for some $p \in[1,2)$.

Of course, (1.1) will be defined as limit of Riemann sums. In order to do so, set, for all $0 \leqslant s \leqslant t \leqslant 1$,

$$
y_{s, t}=f\left(x_{s}\right)\left(x_{t}-x_{s}\right) .
$$

For all $\delta>0$, let $\Pi^{\delta}$ be a family of partitions $0 \leqslant t_{1}^{\delta} \leqslant \cdots \leqslant t_{k^{\delta}}^{\delta} \leqslant 1$ of $[0,1]$ whose meshes decrease to 0 as $\delta$ decreases to 0 . Assume that for all $0<\delta^{\prime}<\delta, \Pi^{\delta} \subset \Pi^{\delta^{\prime}}$.

For all $\delta>0$, set

$$
z_{s, t}^{\Pi^{\delta}}=y_{s, t_{j}^{\delta}}+y_{t_{\ell}^{\delta}, t}+\sum_{i=j}^{\ell-1} y_{t_{i}^{\delta}, t_{i+1}^{\delta}},
$$

where $j$ and $\ell$ are such that $\Pi^{\delta} \cap(s, t)=\left\{t_{j}^{\delta}, \ldots, t_{\ell}^{\delta}\right\}$.

Proposition 1. Under Assumption 2, $z_{s, t}^{\Pi^{\delta}}$ admits a limit denoted by $z_{s, t}$ for all $0 \leqslant s \leqslant t \leqslant 1$. Furthermore, $(s, t) \in \Delta^{+} \mapsto z_{s, t}$ is continuous, and $z_{s, u}+z_{u, t}=z_{s, t}$ (Chasles' relation) for all $0 \leqslant s \leqslant u \leqslant t \leqslant 1$.

Finally, there exists some constant $K$ depending only on $f, p$ and $\omega(0,1)$ such that $\left|z_{s, t}\right|^{p} \leqslant K \omega(s, t)$ for all $(s, t) \in \Delta^{+}$. This implies that $z$ has finite p-variation.

Thus, one may define $\int_{s}^{t} f\left(x_{r}\right) \mathrm{d} x_{r}$ to be $z_{s, t}$. The proof relies on the following Lemmas.

Lemma 1. There exists a constant $C$ depending only on $f$ such that for all $0 \leqslant$ $s \leqslant u \leqslant t \leqslant 1$,

$$
\left|y_{s, t}-y_{s, u}-y_{u, t}\right| \leqslant C \omega(s, t)^{\theta}, \text { with } \theta=\frac{1+\alpha}{p}>1
$$


Proof. Since $f$ is Hölder continuous and $x$ satisfies (2.1), it is easily established that, for $C=\sup _{x \neq y}|f(x)-f(y)| /|x-y|^{\alpha}$,

$$
\begin{array}{r}
\left|y_{s, t}-y_{s, u}-y_{u, t}\right| \leqslant\left|f\left(x_{u}\right)-f\left(x_{s}\right)\right|\left|x_{t}-x_{u}\right| \leqslant C\left|x_{u}-x_{s}\right|^{\alpha}\left|x_{t}-x_{u}\right| \\
\leqslant C \omega(s, u)^{\alpha / p} \omega(u, t)^{1 / p} \leqslant C \omega(s, t)^{(1+\alpha) / p} .
\end{array}
$$

Hence (2.4) is proved.

Lemma 2. Let $0 \leqslant s \leqslant t \leqslant 1$, and let $s<t_{1} \leqslant \ldots \leqslant t_{k}<t$ be a partition of $(s, t)$. Then, if $k \geqslant 2$, there exists an integer $\ell$ in $\{1,2, \ldots, k\}$ such that

$$
\omega\left(t_{\ell-1}, t_{\ell+1}\right) \leqslant \frac{2}{k} \omega(s, t)
$$

with the convention that $t_{0}=s$ and $t_{k+1}=t$.

Proof. The result is clear if $k=2$, since $\omega\left(t_{1}, t_{2}\right) \leqslant \omega(s, t)$. Assume that $k \geqslant$ 3. As $\omega$ is super-additive, $\sum_{i=1}^{k} \omega\left(t_{i-1}, t_{i+1}\right) \leqslant 2 \omega(s, t)$. So, at least one of the $\omega\left(t_{i-1}, t_{i+1}\right)$ 's is smaller than $2 \omega(s, t) / k$.

Proof of Proposition 1. Fix $\delta>0$ and $0 \leqslant s \leqslant t \leqslant 1$. We have $\Pi^{\delta} \cap(s, t)=$ $\left\{t_{j}^{\delta}, \ldots, t_{\ell}^{\delta}\right\}$.

If $\Pi^{\delta} \cap(s, t)=\emptyset$, then $z_{s, t}^{\Pi^{\delta}}=y_{t_{i}^{\delta}, t_{i+1}^{\delta}}$, where the integer $i$ is such that $[s, t] \subset$ $\left[t_{i}^{\delta}, t_{i+1}^{\delta}\right]$.

If $\Pi^{\delta} \cap(s, t)$ contains at least one point, then we choose an integer $k$ such that $j \leqslant k \leqslant \ell$, and we construct a new partition

$$
\Pi=\left\{t_{j}^{\delta}, \ldots, t_{k-1}^{\delta}, t_{k+1}^{\delta}, \ldots, t_{\ell}^{\delta}\right\}
$$

by suppressing the point $t_{k}^{\delta}$. We use the convention that $t_{j-1}^{\delta}=s$ and $t_{\ell+1}^{\delta}=t$.

According to Lemma 2, the point $t_{k}^{\delta}$ is chosen so that

$$
\omega\left(t_{k-1}, t_{k+1}\right) \leqslant \frac{2}{\left|\Pi^{\delta} \cap(s, t)\right|} \omega(s, t) .
$$

Thus, using the previous notations,

$$
z_{s, t}^{\Pi^{\delta}}=z_{s, t}^{\Pi}+y_{t_{k-1}^{\delta},} t_{k}^{\delta}+y_{t_{k}^{\delta}, t_{k+1}^{\delta}}-y_{t_{k-1}^{\delta}, t_{k+1}^{\delta}} .
$$

With Lemma 1,

$$
\left|z_{s, t}^{\Pi^{\delta}}-z_{s, t}^{\Pi}\right| \leqslant C \omega\left(t_{k-1}, t_{k+1}\right)^{\theta} \leqslant C\left(\frac{2}{\left|\Pi^{\delta} \cap(s, t)\right|}\right)^{\theta} \omega(s, t)^{\theta}
$$


for a constant $C$ which is equal to the Hölder norm of $f$.

Suppressing a carefully chosen point in $\Pi$, and reiterating the process, one easily obtains that

$$
\left|z_{s, t}^{\Pi^{\delta}}-y_{s, t}\right| \leqslant K \omega(s, t)^{\theta}+\left|y_{s, t}-y_{t_{i(\delta)}^{\delta}, t_{i^{\prime}(\delta)}^{\delta}}\right|,
$$

where $K=C+2^{\theta} C \sum_{n \geqslant 1} 1 / n^{\theta}$, and $i$ and $i^{\prime}$ are such that $\left[t_{i(\delta)}^{\delta}, t_{i^{\prime}(\delta)}^{\delta}\right]$ is the smallest interval containing $[s, t]$. In particular, $t_{i(\delta)}^{\delta}$ increases to $s$ and $t_{i^{\prime}(\delta)}^{\delta}$ decreases to $t$ as $\delta$ decreases to 0 .

Let $0 \leqslant s \leqslant u \leqslant t \leqslant 1$. Set $\Pi^{\delta} \cap(s, u)=\left\{t_{j}^{\delta}, \ldots, t_{k}^{\delta}\right\}$ and $\Pi^{\delta} \cap[u, t)=$ $\left\{t_{j^{\prime}}^{\delta}, \ldots, t_{k^{\prime}}^{\delta}\right\}$. So,

$$
z_{s, u}^{\Pi^{\delta}}+z_{u, t}^{\Pi^{\delta}}=z_{s, t}^{\Pi^{\delta}}-\left(y_{t_{k}^{\delta}, t_{j^{\prime}}^{\delta}}-y_{t_{k}^{\delta}, u}-y_{u, t_{j^{\prime}}^{\delta}}\right)
$$

As $f$ is bounded, $\left|y_{r, r^{\prime}}\right| \leqslant\|f\|_{\infty} \omega\left(r, r^{\prime}\right)^{1 / p} \underset{\left|r^{\prime}-r\right| \rightarrow 0}{\longrightarrow} 0$. Moreover, $t_{k}^{\delta} \underset{\delta \rightarrow 0}{\longrightarrow} u$ and $t_{j^{\prime}}^{\delta} \underset{\delta \rightarrow 0}{\longrightarrow} u$. Set $Z_{t}^{\delta}=z_{0, t}^{\Pi^{\delta}}$. With (2.6), the inequality $\left|y_{r, r^{\prime}}\right| \leqslant\|f\|_{\infty} \omega\left(r, r^{\prime}\right)^{1 / p}$ and the continuity of $\omega$ near its diagonal, it is easily proved that $\left(Z^{\delta}\right)_{\delta>0}$ satisfies the conditions of the Ascoli theorem, i.e., for any $\kappa>0$, there exists some $\eta>0$ such that $\sup _{|t-s|<\eta}\left|Z_{t}^{\delta}-Z_{s}^{\delta}\right| \leqslant \kappa$. Thus, there exists a subsequence of $\left(Z^{\delta}\right)_{\delta>0}$ which converges uniformly to some continuous function $Z$ on $[0,1]$.

One could set $z_{s, t}=Z_{t}-Z_{s}$. Again with (2.6),$z_{s, t}^{\Pi^{\delta}}=z_{0, t}^{\Pi^{\delta}}-z_{0, s}^{\Pi^{\delta}}+\left(y_{t_{k}^{\delta}, t_{k+1}^{\delta}}-y_{t_{k}^{\delta}, s}-\right.$ $\left.y_{s, t_{k+1}^{\delta}}\right)$, where $t_{k}^{\delta}$ and $t_{k+1}^{\delta}$ are two adjacent points of $\Pi^{\delta}$ such that $s \in\left(t_{k}^{\delta}, t_{k+1}^{\delta}\right]$. Hence, $z_{s, t}^{\Pi^{\delta}}$ converges to $z_{s, t}$ for all $(s, t) \in \Delta^{+}$. Hence, it follows from (2.6) that $z$ satisfies the Chasles relation: $z_{s, t}=z_{s, u}+z_{u, t}$ for all $0 \leqslant s \leqslant u \leqslant t \leqslant 1$. Besides, from the continuity of $Z,(s, t) \in \Delta^{+} \mapsto z_{s, t}$ is continuous.

Let $\widetilde{Z}$ be another limit of the sequence $\left(Z^{\delta}\right)_{\delta>0}$, and set $\widetilde{z}_{s, t}=\widetilde{Z}_{t}-\widetilde{Z}_{s}$ for all $(s, t) \in \Delta^{+}$. As for $z, \widetilde{z}$ also satisfies the Chasles relation, and so is $\Delta z=\widetilde{z}-z$. However, $|\Delta z| \leqslant 2 K \omega(s, t)^{\theta}$. For any partition $\Pi=\left\{t_{1}, \ldots, t_{k}\right\}$ of $[s, t]$,

$$
\begin{aligned}
\left|\Delta z_{s, t}\right| & \leqslant \sum_{i=1}^{k-1}\left|\Delta z_{t_{i}, t_{i+1}}\right| \leqslant 2 K \sum_{=1}^{k-1} \omega\left(t_{i}, t_{i+1}\right)^{\theta} \\
& \leqslant 2 K \omega(s, t) \sup _{i=1, \ldots, k} \omega\left(t_{i}, t_{i+1}\right)^{\theta-1} \underset{\operatorname{mesh}(\Pi) \rightarrow 0}{\longrightarrow} 0 .
\end{aligned}
$$

So, the limit of $\left(z_{s, t}^{\Pi^{\delta}}\right)_{\delta>0}$ is unique.

With (2.5) and the boundedness of $f,\left|z_{s, t}\right| \leqslant K \omega(s, t)^{\theta}+\left|y_{s, t}\right|$. But $\left|y_{s, t}\right| \leqslant$ $\|f\|_{\infty}\left|x_{s, t}\right| \leqslant\|f\|_{\infty} \omega(s, t)^{1 / p}$. Thus, $\left|z_{s, t}\right|^{p} \leqslant\left(K \omega(0,1)^{\alpha}+\|f\|_{\infty}^{p}\right) \omega(s, t)$ and $z$ is of finite $p$-variation. The proposition is then proved. 


\subsection{The semi-norm of $p$-variation}

In the preceding proof, the regularity of $x$ plays in fact no role. The only condition required is (2.2). Note that (2.2) implies that for any partition $\Pi=\left\{t_{0}, \ldots, t_{k}\right\}$ of $[s, t]$,

$$
\sum_{i=0}^{k-1}\left|x_{t_{i+1}}-x_{t_{i}}\right|^{p} \leqslant \sum_{i=0}^{k-1} \omega\left(t_{i}, t_{i+1}\right) \leqslant \omega(s, t) .
$$

Define the semi-norm of $p$-variation by

$$
\operatorname{Var}_{p,[s, t]}(x)=\sup _{\substack{\Pi=\left\{t_{0}, \ldots, t_{k}\right\} \\ \text { partition of }[\mathrm{s}, \mathrm{t}]}}\left(\sum_{i=0}^{k-1}\left|x_{t_{i+1}}-x_{t_{i}}\right|^{p}\right)^{1 / p}
$$

Remark 1. When one considers $x(t)=t$ and $p>1$, it is immediate that for any partition $0 \leqslant t_{0} \leqslant \cdots \leqslant t_{k} \leqslant 1$, the following inequality holds: $\sum_{i=0}^{k-1}\left|t_{i+1}-t_{i}\right|^{p} \leqslant$ $\sup _{i=0, \ldots, k-1}\left|t_{i+1}-t_{i}\right|^{p-1}$. The later quantity converges to 0 with the mesh of the partition. But $\operatorname{Var}_{p,[s, t]}(x)=1$. This means that in the definition of the $p$-variation, we have really to consider a supremum on all the partitions, and not only on those whose mesh converges to 0 .

An interesting property of the $p$-variation is that as soon as $\operatorname{Var}_{p,[s, t]}(x)$ is finite, then $\operatorname{Var}_{q,[s, t]}(x) \leqslant \operatorname{Var}_{p,[s, t]}(x)$ for all $q \geqslant p$. In other words, any function of finite $p$-variation is of finite $q$-variation for all $q \geqslant p$.

Inequality (2.2) in Assumption 2 means that $\operatorname{Var}_{p,[s, t]}(x) \leqslant \omega(s, t)$ for all $0 \leqslant s \leqslant t \leqslant 1$. On the other hand, we know that $\operatorname{Var}_{p,[s, u]}(x)+\operatorname{Var}_{p,[u, t]}(x) \leqslant$ $\operatorname{Var}_{p,[s, t]}(x)$.

Although $\operatorname{Var}_{p,[0,1]}$ is only a semi-norm, $\operatorname{Var}_{p,[0,1]}(\cdot)+\|\cdot\|_{\infty}$ is a norm on the space of continuous function. However, the space of continuous functions with this norm is not separable.

Set for $(s, t) \in \Delta^{+}$and two continuous functions $x$ and $y$,

$$
\delta_{p,[s, t]}(x, y)=\operatorname{Var}_{p,[s, t]}(x-y),
$$

and $\delta_{p,[s, t]}(x)=\delta_{p,[s, t]}(x, 0)$. Set also $\delta_{p}(x, y)=\delta_{p,[0,1]}(x, y)$ and $\delta_{p}(x)=\delta_{p,[0,1]}(x)$. Note that $\delta_{p,[0,1]}$ is a not a distance, excepted when restricted to functions for which $x_{0}$ is fixed.

Let $x$ be a function such that $\delta_{p}(x)$ is finite. If there exists a function $\omega: \Delta^{+} \rightarrow$ $\mathbb{R}_{+}$satisfying Assumption 1 and such that

$$
\left|x_{t}-x_{s}\right|^{p} \leqslant \omega(s, t)
$$

for all $(s, t) \in \Delta^{+}$, then $x$ is said to be of finite p-variation controlled by $\omega$. It is clear that the function $\omega$ defined by $\omega(s, t)=\delta_{p,[s, t]}(x)^{p}$ satisfies Assumptions 1 and that for all $(s, t) \in \Delta^{+},\left|x_{t}-x_{s}\right|^{p} \leqslant \omega(s, t)$.

The following lemma is related to sequences of functions of finite $p$-variation. 
Lemma 3. Let $\left(x^{n}\right)_{n \in \mathbb{N}}$ be a sequence of functions of finite p-variation and let $x$ be a function of finite $p$-variation such that $\delta_{p}\left(x^{n}, x\right)$ converges to 0 . Then there exists a subsequence $\left(x^{n_{k}}\right)_{k \in \mathbb{N}}$ and some function $\omega$ satisfying Assumption 1 such that $x^{n_{k}}$ and $x$ are of finite $p$-variation controlled by $\omega$. Moreover, for any $\varepsilon>0$, there exists an integer $k$ for which

$$
\forall \ell \geqslant k, \delta_{p,[s, t]}\left(x^{n_{\ell}}, x\right) \leqslant \varepsilon \omega(s, t) \text { for all }(s, t) \in \Delta^{+} .
$$

Proof. There exists a subsequence $\left(n_{k}\right)_{k \in \mathbb{N}}$ such that $\delta_{p}\left(x, x^{n_{k}}\right) \leqslant 4^{-k}$. Hence, we set

$$
\omega(s, t)=2^{p-1}\left(\delta_{p,[s, t]}(x)^{p}+\sum_{k=0}^{+\infty} 2^{k} \delta_{p,[s, t]}\left(x^{n_{k}}, x\right)^{p}\right) .
$$

By our choice of the subsequence, this function $\omega$ is well defined for all $(s, t) \in$ $\Delta^{+}$. As $\delta_{p,[s, t]}\left(x^{n}, x\right) \leqslant \delta_{p}\left(x^{n}, x\right) \underset{n \rightarrow \infty}{\longrightarrow} 0$, and $\delta_{p,[s, t]}\left(x^{n}, x\right)$ is continuous near the diagonal, $(s, t) \mapsto \sum_{k=0}^{+\infty} 2^{k} \delta_{p,[s, t]}\left(x^{n_{k}}, x\right)^{p}$ is continuous near the diagonal. Similarly, $(s, t) \mapsto \delta_{p,[s, t]}\left(x^{n}\right)^{p}$ is continuous near the diagonal. Clearly, $\omega$ is super-additive and satisfies Assumption 1 .

Since $\delta_{p,[s, t]}\left(x^{n_{k}}\right)^{p} \leqslant 2^{p-1} \delta_{p,[s, t]}\left(x^{n_{k}}, x\right)^{p}+2^{p-1} \delta_{p,[s, t]}(x)^{p}, x$ and all of the $x^{n_{k}}$ 's are controlled by $\omega$. Furthermore,

$$
\delta_{p,[s, t]}\left(x^{n_{k}}, x\right)^{p} \leqslant \frac{1}{2^{k}} \omega(s, t),
$$

and the lemma is proved.

The proof of the following Lemma is straightforward.

Lemma 4 (A convergence and compactness criterion). Let $q$ be a real number greater than $p$. Then,

$$
\operatorname{Var}_{q,[0,1]}\left(x-x^{n}\right)^{q} \quad \leqslant 2^{p} \sup _{r \in[0,1]}\left(x_{r}-x_{r}^{n}\right)^{q-p}\left(\operatorname{Var}_{p,[0,1]}(x)^{p}+\operatorname{Var}_{p,[0,1]}\left(x^{n}\right)^{p}\right) .
$$

Moreover, if $\left(x^{n}\right)_{n \in \mathbb{N}}$ converges pointwise to $x$, then

$$
\operatorname{Var}_{p,[0,1]}(x) \leqslant \liminf _{n \in \mathbb{N}} \operatorname{Var}_{p,[0,1]}\left(x^{n}\right) .
$$

Hence, to prove that $x^{n}$ converges to $x$ in $q$-variation, we have only to prove that $x^{n}$ converges uniformly to $x$ and that $\sup _{n \in \mathbb{N}} \operatorname{Var}_{p,[0,1]}\left(x^{n}\right)$ is finite for some $p<q$.

Thus, if $\left(x^{n}\right)_{n \in \mathbb{N}}$ is equi-continuous, uniformly bounded, and the sequence $\left(\operatorname{Var}_{p,[0,1]}\left(x^{n}\right)\right)_{n \in \mathbb{N}}$ is also bounded, then there exists a subsequence of $\left(x^{n}\right)_{n \in \mathbb{N}}$ which converges uniformly to a function $x$. With (2.8), $x^{n}$ converges in $q$-variation to $x$ for any $q>p$. 
Remark 2 (Extension of Helly's selection principle). If $\left(x^{n}\right)_{n \in \mathbb{N}}$ is a family of continuous functions uniformly bounded and of finite $p$-variation such that $\left(\operatorname{Var}_{p,[0,1]}\left(x^{n}\right)\right)_{n \in \mathbb{N}}$ is bounded, then there exists a function $x$ of finite $p$-variation and a subsequence $\left(x^{n_{k}}\right)_{k \in \mathbb{N}}$ such that $x^{n_{k}}$ converges pointwise to $x$. But $x$ is not necessarily continuous. This may be seen as an extension of Helly's selection principle (see [7, Theorem 6.1]).

\subsection{Continuity}

For any bounded and $\alpha$-Hölder continuous function $f$ with a Hölder constant $\alpha>p-1$, we have constructed a map $\mathfrak{K}_{f}: x \mapsto z$, where $x$ is a function on $[0,1]$ with finite $p$-variation (with $1 \leqslant p<2$ ) and $z$ is the function $\left(\int_{s}^{t} f\left(x_{r}\right) \mathrm{d} x_{r}\right.$; $0 \leqslant s \leqslant t \leqslant 1)$

We have seen in Proposition 1 that $\mathfrak{K}_{f}(x)$ is also of finite $p$-variation.

We are now interested in the continuity of $\mathfrak{K}_{f}$. Let $x$ and $\widetilde{x}$ be two functions of finite $p$-variation, both satisfying Assumption 2 with respect to the same $\omega$.

Proposition 2. Assume that there exists some $\varepsilon>0$ such that for all $0 \leqslant s \leqslant$ $t \leqslant 1$,

$$
\left|\left(x_{t}-\widetilde{x}_{t}\right)-\left(x_{s}-\widetilde{x}_{s}\right)\right|^{p} \leqslant \varepsilon \omega(s, t),
$$

and that $x_{0}=\widetilde{x}_{0}$. Then there exists a function $\kappa(\varepsilon)$ decreasing to 0 as $\varepsilon$ decreases to 0 and depending only on $f$ and $p$ such that

$$
\left|\mathfrak{K}_{f}(x)_{s, t}-\mathfrak{K}_{f}(\widetilde{x})_{s, t}\right| \leqslant \kappa(\varepsilon) \omega(s, t)^{1 / p}
$$

for all $(s, t) \in \Delta^{+}$.

Proof. The proof is similar to the one of Proposition 1. Using the same notations, define $z_{s, t}^{\Pi^{\delta}}$ and $\widetilde{z}_{s, t}^{\Pi^{\delta}}$. As previously, we create a new partition $\Pi$ by suppressing a carefully chosen point of $\Pi^{\delta}$. Hence, to estimate

$$
\left|\left(z_{s, t}^{\Pi^{\delta}}-\widetilde{z}_{s, t}^{\Pi^{\delta}}\right)-\left(z_{s, t}^{\Pi}-\widetilde{z}_{s, t}^{\Pi}\right)\right|,
$$

we have only to estimate, for all $u \in[s, t]$,

$$
\Delta=\left|\left(y_{s, t}-y_{s, u}-y_{u, t}\right)-\left(\widetilde{y}_{s, t}-\widetilde{y}_{s, u}-\widetilde{y}_{u, t}\right)\right|,
$$

with $y_{s, t}=f\left(x_{s}\right)\left(x_{t}-x_{s}\right)$ and $\widetilde{y}_{s, t}=f\left(\widetilde{x}_{s}\right)\left(\widetilde{x}_{t}-\widetilde{x}_{s}\right)$. Thus,

$$
\begin{aligned}
|\Delta| \leqslant & \left|\left(f\left(x_{u}\right)-f\left(x_{s}\right)\right)\left(x_{t}-x_{u}\right)-\left(f\left(\widetilde{x}_{u}\right)-f\left(\widetilde{x}_{s}\right)\right)\left(\widetilde{x}_{t}-\widetilde{x}_{u}\right)\right| \\
\leqslant & \left|f\left(x_{u}\right)-f\left(x_{s}\right)\right|\left|x_{t}-\widetilde{x}_{t}-\left(x_{u}-\widetilde{x}_{u}\right)\right| \\
& +\left|f\left(x_{u}\right)-f\left(x_{s}\right)-f\left(\widetilde{x}_{u}\right)+f\left(\widetilde{x}_{s}\right)\right|\left|\widetilde{x}_{t}-\widetilde{x}_{u}\right| .
\end{aligned}
$$


Now, if $C$ denotes the $\alpha$-Hölder constant of $f$, we remark that

$$
\left|f\left(x_{u}\right)-f\left(x_{s}\right)-f\left(\widetilde{x}_{u}\right)+f\left(\widetilde{x}_{s}\right)\right| \leqslant 2 \varepsilon^{\alpha / p} C \omega(0,1)^{1 / p}
$$

and that

$$
\left|f\left(x_{u}\right)-f\left(x_{s}\right)-f\left(\widetilde{x}_{u}\right)+f\left(\widetilde{x}_{s}\right)\right| \leq 2 C \omega(s, t)^{\alpha / p} .
$$

Choosing $\beta \in(0,1)$ such that $\beta \alpha+1>p$, one gets

$$
|\Delta| \leqslant C \varepsilon^{1 / p} \omega(s, t)^{(1+\alpha) / p}+2 C \varepsilon^{\alpha(1-\beta) / p} \omega(0,1)^{(1-\beta) / p} \omega(s, t)^{(\alpha \beta+1) / p} .
$$

Hence, as in the proof of Proposition 1,

$$
\left|z_{s, t}^{\Pi^{\delta}}-\widetilde{z}_{s, t}^{\Pi^{\delta}}-y_{s, t}-\widetilde{y}_{s, t}\right| \leqslant \kappa^{\prime}(\varepsilon) \omega(s, t)^{\theta},
$$

where $\kappa^{\prime}(\varepsilon)$ decreases to 0 with $\epsilon$, and depends only on $f, x_{0}$ and $p$. On the other hand, there exists some function $\kappa^{\prime \prime}$ decreasing to 0 with $\varepsilon$ such that $\left|y_{s, t}-\widetilde{y}_{s, t}\right| \leqslant$ $\kappa^{\prime \prime}(\varepsilon) \omega(s, t)^{1 / p}$. In the limit, if $\kappa=\kappa^{\prime} \omega(0,1)^{(\theta-1) / p}+\kappa^{\prime \prime}$,

$$
\left|z_{s, t}-\widetilde{z}_{s, t}\right|=\left|\mathfrak{K}_{f}(x)_{s, t}-\mathfrak{K}_{f}(\widetilde{x})_{s, t}\right| \leqslant \kappa(\varepsilon) \omega(s, t)^{1 / p}
$$

The Proposition is then proved.

Denote by $\mathcal{G}^{p}\left(\mathbb{R}^{d}\right)$ the space of continuous functions in $\mathcal{C}\left([0,1] ; \mathbb{R}^{d}\right)$ of finite $p$-variation and starting at the same given point $x_{0}$. Denote by $\mathcal{V}^{p}$ the topology that the distance $\delta_{p}$ defines on the space $\mathcal{G}^{p}\left(\mathbb{R}^{d}\right)$.

Corollary 1. Let $f$ be a bounded and $\alpha$-Hölder continuous function, and let $p \in$ $[1,2)$ be such that $\alpha>p-1$. Let $\left(x_{n}\right)_{n \in \mathbb{N}}$ be a sequence of continuous functions in $\mathcal{G}^{p}\left(\mathbb{R}^{d}\right)$ converging in $\mathcal{V}^{p}$ to a function $x$ in $\mathcal{G}^{p}\left(\mathbb{R}^{d}\right)$. Then $\mathfrak{K}_{f}\left(x_{n}\right) \in \mathcal{G}^{p}\left(\mathbb{R}^{d}\right)$ converges in $\mathcal{V}^{p}$ to $\mathfrak{K}_{f}(x)$. Thus, $x \mapsto \mathfrak{K}_{f}(x)$ is continuous with respect to $\delta_{p}$.

Proof. Using Lemma 3, there exists a function $\omega: \Delta^{+} \rightarrow \mathbb{R}_{+}$satisfying Assumption 1 that controls $x$ and $x^{n}$ (or maybe a subsequence of it) and such that for any $\varepsilon>0$, there exists some integer $n_{\varepsilon}$ for which

$$
\left|x_{t}-x_{s}-\left(x_{t}^{n}-x_{s}^{n}\right)\right|^{p} \leqslant \varepsilon \omega(s, t)
$$

for every $n \geqslant n_{\varepsilon}$ and all $(s, t) \in \Delta^{+}$. From Proposition 2 , for all $(s, t) \in \Delta^{+}$and any $n \in \mathbb{N}$,

$$
\left|\mathfrak{K}_{f}(x)_{s, t}-\mathfrak{K}_{f}\left(x^{n}\right)_{s, t}\right| \leqslant \kappa(\varepsilon) \omega(s, t)^{1 / p},
$$

with $\kappa(\varepsilon) \underset{\varepsilon \rightarrow 0}{\longrightarrow} 0$. This function $\kappa$ depends only on $f$ and $p$. As $\omega$ is bounded on $\Delta^{+}$,

$$
\operatorname{Var}_{p,[0,1]}\left(\mathfrak{K}_{f}(x)-\mathfrak{K}_{f}\left(x^{n}\right)\right)^{p} \underset{n \rightarrow \infty}{\longrightarrow} 0 .
$$

The previous convergences are proved at least along a subsequence, but using the way Lemma 3 is proved, the limit of $\mathfrak{K}_{f}\left(x^{n}\right)$ is in fact unique. The Corollary is then proved. 
As an application, let $\Pi^{\delta}=\left\{t_{i}^{\delta} \mid 0 \leqslant t_{0}^{\delta} \leqslant \cdots \leqslant t_{k^{\delta}}^{\delta} \leqslant 1\right\}$ be a family of partitions of $[0,1]$ whose meshes go to 0 with $\delta$. Then it is easily seen that the piecewise linear approximation $x^{\delta}$ of some path $x \in \mathcal{G}^{q}\left(\mathbb{R}^{d}\right)$ for some $q \in[1,2)$ given by

$$
x_{t}^{\delta}=x_{t_{i}^{\delta}}+\frac{t-t_{i}^{\delta}}{t_{i+1}^{\delta}-t_{i}^{\delta}}\left(x_{t_{i+1}^{\delta}}-x_{t_{i}^{\delta}}\right) \text { when } t \in\left[t_{i}^{\delta}, t_{i+1}^{\delta}\right]
$$

converges uniformly to $x$.

Let $0 \leqslant s_{0} \leqslant \ldots \leqslant s_{\ell} \leqslant 1$ be a partition of $[0,1]$. Then,

$$
\begin{aligned}
\sum_{i=1}^{\ell-1}\left|x_{s_{i+1}}^{\delta}-x_{s_{i}}^{\delta}\right|^{q}= & \sum_{j=0}^{k^{\delta}-1} \sum_{i \text { s.t. }} \mid x_{i} \in\left[t_{j}^{\delta}, t_{j+1}^{\delta}\right] \\
& +\sum_{j=0}^{k_{i+1}^{\delta}-1} \sum_{\text {i s.t. } t_{j}^{\delta} \in\left(s_{i}, s_{i+1}\right)}\left|x_{s_{i+1}}^{\delta}-x_{s_{i}}^{\delta}\right|^{q} .
\end{aligned}
$$

However,

$$
\sum_{i \text { s.t. } s_{i} \in\left[t_{j}^{\delta}, t_{j+1}^{\delta}\right]}\left|x_{s_{i+1}}^{\delta}-x_{s_{i}}^{\delta}\right|^{q} \leqslant\left|x_{t_{j+1}}-x_{t_{j}}\right|^{q}
$$

and if $i$ is such that $t_{j}^{\delta} \in\left(s_{i}, s_{i+1}\right)$ for a given $j$, then

$$
\left|x_{s_{i+1}}^{\delta}-x_{s_{i}}^{\delta}\right|^{q} \leqslant 2^{q-1}\left|x_{t_{j+1}^{\delta}}-x_{t_{j}^{\delta}}\right|^{q}+2^{q-1}\left|x_{t_{j}^{\delta}}-x_{t_{j-1}^{\delta}}\right|^{q} .
$$

In the previous inequality, we set if necessary, $t_{-1}^{\delta}=0$ and $t_{k^{\delta}+1}^{\delta}=1$. It is now clear that $\operatorname{Var}_{q,[0,1]}\left(x^{\delta}\right) \leqslant 3 \operatorname{Var}_{q,[0,1]}(x)$, and then, from Lemma $4, \operatorname{Var}_{p,[0,1]}\left(x^{\delta}-x\right)$ converges to 0 for all $p>q$. If follows that

$$
\int_{s}^{t} f\left(x_{r}^{\delta}\right) \frac{\mathrm{d} x_{r}^{\delta}}{\mathrm{d} r} \mathrm{~d} r \underset{\delta \rightarrow 0}{\longrightarrow} \int_{s}^{t} f\left(x_{r}\right) \mathrm{d} x_{r}
$$

This convergence holds in fact both in $p$-variation and uniformly.

\subsection{Solving differential equations}

Let $f=\left(f_{1}, \ldots, f_{d}\right)$ be a function from $\mathrm{W}=\mathbb{R}^{m}$ to $\mathrm{W}^{d}$. We are now interested in solving the differential equation

$$
y_{t}=y_{s}+\sum_{i=1}^{d} \int_{s}^{t} f_{i}\left(y_{r}\right) \mathrm{d} x_{r}^{i}
$$

where $x$ is a continuous function of finite $p$-variation, with $p \in[1,2)$. 
Theorem 1. If $f$ is $\alpha$-Hölder continuous with $\alpha>p-1$ and $x$ is in $\mathcal{G}^{p}\left(\mathbb{R}^{d}\right)$, then there exists a solution $y$ in $\mathcal{G}^{p}\left(\mathbb{R}^{m}\right)$ to (2.9). Moreover, if $f$ is bounded, continuous with a bounded derivative which is $\alpha$-Hölder continuous with $\alpha>p-1$, then $y$ with a given initial condition $y_{0}$, is unique. Besides, the map $x \mapsto y=\mathfrak{I}_{f, y_{0}}(x)$ (called the Itô map) is continuous from $\mathcal{G}^{p}\left(\mathbb{R}^{d}\right)$ to $\mathcal{G}^{p}\left(\mathbb{R}^{m}\right)$.

Proof. For two continuous paths $y, x$ of finite $p$-variation, denote by $\mathfrak{L}$ the map defined by

$$
\mathfrak{L}_{s, t}(y, x)=\left[\begin{array}{c}
\widehat{y}_{s, t} \\
\widehat{x}_{s, t}
\end{array}\right]=\int_{s}^{t}\left(\sum_{i=1}^{m}\left[\begin{array}{l}
0 \\
0
\end{array}\right] \mathrm{d} y_{r}^{i}+\sum_{i=1}^{d}\left[\begin{array}{c}
f_{i}\left(y_{r}\right) \\
1
\end{array}\right] \mathrm{d} x_{r}^{i}\right)
$$

for any $(s, t) \in \Delta^{+}$. Clearly, $\widehat{x}=x$. Define also $\mathfrak{I}(y, x)=\widehat{y}$. For any integer $n \geqslant 1$, set $y^{n}=\mathfrak{I}\left(y^{n-1}, x\right)$. Of course, if $y^{n}$ converges to some function $y$ in $\mathcal{G}^{p}\left(\mathbb{R}^{m}\right)$, then $y$ is solution to (2.9).

Step 1: Existence. Assume that two paths $x$ and $y$ of finite $p$-variation are controlled respectively by $\omega$ and $\gamma \omega$ on a time interval $[S, T]$, for some constant $\gamma>0$.

A slight modification of the proof of Proposition 1 shows that there exists some constant $K$, depending only on $f$ and $p$, such that

$$
\left|\mathfrak{I}_{s, t}(y, x)-f\left(y_{s}\right) x_{s, t}\right| \leqslant \gamma^{\alpha / p} K \omega(s, t)^{\theta} \text { for all } S \leqslant s \leqslant t \leqslant T,
$$

with $\theta=\frac{1+\alpha}{p}>1$. Hence,

$$
\left|\mathfrak{I}_{s, t}(y, x)\right| \leqslant\left(K \gamma^{\alpha / p} \omega(S, T)^{\theta-1 / p}+\|f\|_{\infty}\right) \omega(s, t)^{1 / p} .
$$

If $\gamma=2^{p}\|f\|_{\infty}^{p}$, and $S$ and $T$ are close enough so that

$$
\omega(S, T)^{\theta-1 / p} K \gamma^{\alpha / p} \leqslant\|f\|_{\infty},
$$

we have proved that on $[S, T], \mathfrak{I}(y, x)$ is of finite $p$-variation controlled by $\gamma \omega(s, t)$.

Thus, one may construct a finite number $N$ of intervals $\left[T_{i}, T_{i+1}\right]$ such that $T_{0} \leqslant T_{1} \leqslant \cdots \leqslant T_{N}$ and $\omega\left(T_{i}, T_{i+1}\right)^{\theta-1 / p} \leqslant 2^{-\alpha} K^{-1}\|f\|_{\infty}^{1-\alpha}$.

From a function $y^{0}$ of finite $p$-variation controlled by $\gamma \omega$, one may recursively construct functions $y^{n}$ by setting $y_{s, t}^{n}=\mathfrak{I}\left(y^{n-1}, x\right)_{s, t}$ and $y_{0}^{n}=y_{0}$, where $y_{0}$ is a given point in $\mathbb{R}^{m}$.

On each interval $\left[T_{i}, T_{i+1}\right], y^{n}$ is of finite $p$-variation controlled by $\gamma \omega$. From the convexity inequality, $\left|y_{s, t}^{n}\right|^{p} \leqslant N^{p-1} \gamma \omega(s, t)$ for all $(s, t) \in \Delta^{+}$.

So, $\left(y_{t}^{n} ; 0 \leqslant t \leqslant 1\right)_{n \in \mathbb{N}}$ is equi-continuous, bounded, and according to Ascoli's theorem and Lemma 4 , there exists some $y$ of finite $p$-variation such that a subsequence of $\left(y^{n}\right)_{n \in \mathbb{N}}$ converges to $y$ in $q$-variation for some $q>p$. But $y \mapsto \mathfrak{I}(y, x)$ 
is also continuous on $\mathcal{G}^{q}\left(\mathbb{R}^{m}\right)$. So, we deduce that $y$ is solution to (2.9) with the initial condition $y_{0}$.

Step 2: Uniqueness. In this step, assume that $f$ is continuous, bounded with a bounded $\alpha$-Hölder continuous derivative with $\alpha>p-1$.

Let $y$ and $\widehat{y}$ be two paths of finite $p$-variation controlled by $\omega$ and starting from the same point, that is $y_{0}=\widehat{y}_{0}$. Assume also that $x$ is of finite $p$-variation controlled by $\omega$, and that $y-\widehat{y}$ is of finite $p$-variation controlled by $\gamma \omega$ for some $\gamma>0$. It is clear that $\gamma$ may be chosen smaller than $2^{p}$.

We are interested in $\mathfrak{I}(y, x)-\mathfrak{I}(\widehat{y}, x)$. With our construction, this difference is approximated by $\sum_{i=1}^{k-1}\left(f\left(y_{t_{i}}\right)-f\left(\widehat{y}_{t_{i}}\right)\right) x_{t_{i}, t_{i+1}}$ on some partitions $\Pi=$ $\left\{t_{i} \mid 0 \leqslant t_{1} \leqslant \ldots \leqslant t_{k} \leqslant 1\right\}$ whose meshes go to 0 . We follow the proof of Proposition 1 and we set for all $s \leqslant u \leqslant t$,

$$
\begin{aligned}
\varepsilon_{s, u, t}= & \left|\left(f\left(y_{s}\right)-f\left(\widehat{y}_{s}\right)\right) x_{s, t}-\left(f\left(y_{s}\right)-f\left(\widehat{y}_{s}\right)\right) x_{s, u}-\left(f\left(y_{u}\right)-f\left(\widehat{y}_{u}\right)\right) x_{u, t}\right| \\
= & \left|\left(f\left(y_{s}\right)-f\left(y_{u}\right)-\left(f\left(\widehat{y}_{s}\right)-f\left(\widehat{y}_{u}\right)\right)\right) x_{u, t}\right| \\
\leqslant & \mid \int_{0}^{1} \nabla f\left(y_{s}+\tau\left(\widehat{y}_{s}-y_{s}\right)\right) \cdot\left(\widehat{y}_{s}-y_{s}\right) \mathrm{d} \tau \\
& -\int_{0}^{1} \nabla f\left(y_{u}+\tau\left(\widehat{y}_{u}-y_{u}\right)\right) \cdot\left(\widehat{y}_{u}-y_{u}\right) \mathrm{d} \tau \mid \omega(s, t)^{1 / p} .
\end{aligned}
$$

But, as $\nabla f$ is $\alpha$-Hölder continuous, there exists some constant $C$ such that

$$
\begin{aligned}
\varepsilon_{s, u, t} \leqslant & \omega(s, t)^{1 / p}\left(\|\nabla f\|_{\infty}\left(\widehat{y}_{s}-y_{s}-\widehat{y}_{u}+y_{u}\right)\right. \\
& \left.+C\left|\widehat{y}_{u}-y_{u}\right| \int_{0}^{1}\left|y_{s}-y_{u}+\tau\left(\widehat{y}_{s}-y_{s}-\widehat{y}_{u}+y_{u}\right)\right|^{\alpha} \mathrm{d} \tau\right) \\
\leqslant & \omega(s, t)^{1 / p}\left(\|\nabla f\|_{\infty} \gamma^{1 / p} \omega(s, t)^{1 / p}+C \gamma^{1 / p} \omega(0, u)^{1 / p} \omega(s, u)^{\alpha / p}\right. \\
& \left.+C \gamma^{(1+\alpha) / p} \omega(0, u)^{1 / p} \omega(s, u)^{\alpha / p}\right) \\
\leqslant & \omega(s, t)^{(1+\alpha) / p} \gamma^{1 / p}\left(C_{1}+C_{2} \gamma^{\alpha / p}\right)
\end{aligned}
$$

where $C_{1}$ and $C_{2}$ depend only on $f, \omega, \alpha$ and $p$. We have also remarked that $\gamma$ may be chosen smaller than $2^{p}$, so $\varepsilon_{s, u, t} \leqslant \omega(s, t)^{(1+\alpha) / p} \gamma^{1 / p}\left(C_{1}+2^{\alpha} C_{2}\right)$.

Following the proof of Proposition 1, for all $(s, t) \in \Delta^{+}$, there exist some constant $K$ depending only on $f, \omega, \alpha$ and $p$ such that

$$
\left|\mathfrak{I}_{s, t}(y, x)-\mathfrak{I}_{s, t}(\widehat{y}, x)-\left(f\left(y_{s}\right)-f\left(\widehat{y}_{s}\right)\right) x_{s, t}\right| \leqslant K \gamma^{1 / p} \omega(s, t)^{(1+\alpha) / p} .
$$


On the other hand,

$$
\begin{aligned}
\left|\left(f\left(y_{s}\right)-f\left(\widehat{y}_{s}\right)\right) x_{s, t}\right| & \leqslant \omega(s, t)^{1 / p}\|\nabla f\|_{\infty}\left|y_{0, s}-\widehat{y}_{0, s}\right| \\
& \leqslant \omega(s, t)^{1 / p} \omega(0, s)^{1 / p} \gamma^{1 / p}\|\nabla f\|_{\infty} .
\end{aligned}
$$

From (2.10) and (2.11), one can select a time $T$ small enough depending on $\alpha, p$, $f$ and $\omega$ such that

$$
\left|\mathfrak{I}_{s, t}(y, x)-\mathfrak{I}_{s, t}(\widehat{y}, x)\right| \leqslant \frac{\gamma^{1 / p}}{2} \omega(s, t)^{1 / p} .
$$

In other words, $\mathfrak{I}(y, x)-\mathfrak{I}(\widehat{y}, x)$ is controlled by $2^{-p} \gamma \omega$ on $[0, T]$.

If both $y$ and $\widehat{y}$ are solutions to (2.9), then $\mathfrak{I}(y, x)-\mathfrak{I}(\widehat{y}, x)=y-\widehat{y}$. So, iterating the procedure, one deduces that $y-\widehat{y}$ is controlled by $2^{-n p} \omega$ on the time interval $[0, T]$ for each integer $n$. This proves that $y=\widehat{y}$ on $[0, T]$. Similarly, it is possible to construct iteratively a finite sequence of increasing times $T_{k}$ for $k=1, \ldots, n$ with $T_{1}=0, T_{2}=T$ and such that $T_{n}=1$ and $y=\widehat{y}$ on $\left[T_{k}, T_{k+1}\right]$ as soon as $y_{T_{k}}=\widehat{y}_{T_{k}}$. For that, these times are constructed so that $\omega\left(T_{k}, T_{k+1}\right)$ is smaller than a given constant $c$ small enough, which explains why this set is finite. We deduce that the solution of (2.9) is unique on $[0,1]$.

Step 3: Continuity. Denote by $\mathfrak{I}_{f, y_{0}}$ the map which at $x$ gives the solution $y$ to (2.9) with the given initial condition $y_{0}$.

For a given $y^{0}$, one may iteratively construct for each integer $n \geqslant 1$ a path $y^{n}$ by setting $y^{n}=\mathfrak{I}\left(y^{n-1}, x\right)$. In Step 1 , we have seen that $\left(y^{n}\right)_{n \in \mathbb{N}}$ admits a convergent subsequence, and in Step 2, under stronger hypotheses on $f$, that the limit $\mathfrak{I}_{f, y_{0}}(x)$ of $\left(y^{n}\right)_{n \in \mathbb{N}}$ is unique. Furthermore, if $y^{0}, y^{1}, y^{1}-y^{0}$ and $x$ are of finite $p$-variation controlled by $\omega, y^{n}-y^{n-1}$ are of finite $p$-variation controlled by $2^{-(n-1) p} \omega$. So, $\mathfrak{I}_{f, y_{0}}(x)-y^{n}$ is of finite $p$-variation controlled by $2^{-(n-2) p} \omega$.

Now, consider two paths $x$ and $\widehat{x}$ both of finite $p$-variation controlled by $\omega$, and such that $x-\widehat{x}$ is of finite $p$-variation controlled by $\varepsilon \omega$ for some $\varepsilon>0$. Let $\left(y^{n}\right)_{n \in \mathbb{N}}$ and $\left(\widehat{y}^{n}\right)_{n \in \mathbb{N}}$ be two sequences of functions of finite $p$-variation controlled by $\omega$ with $y^{0}=\widehat{y}_{0}$ and constructed by setting $y^{n}=\mathfrak{I}\left(y^{n-1}, x\right)$ and $\widehat{y}^{n}=\mathfrak{I}\left(\widehat{y}^{n-1}, \widehat{x}\right)$. From Proposition 2 it is clear that for each $n \geqslant 0$, there exists a function $\varphi_{n}(\varepsilon)$ converging to 0 with $\varepsilon$ such that $y^{n}-\widehat{y}^{n}$ is of finite $p$-variation controlled by $\varphi_{n}(\varepsilon) \omega$. But $y-y^{n}$ and $\widehat{y}-\widehat{y}^{n}$ are both of finite $p$-variation controlled by $2^{-(n-1) p} \omega$.

Thus, for all $\eta>0$, there exists $n_{0}$ large enough so that both $\mathfrak{I}_{f, y_{0}}(x)-y^{n}$ and $\mathfrak{I}_{f, y_{0}}(\widehat{x})-\widehat{y}^{n}$ is controlled by $\eta \omega$ for all $n \geqslant n_{0}$. Besides, if $\varepsilon$ is small enough and $x-\widehat{x}$ is controlled by $\varepsilon \omega$, then $y^{n_{0}}-\widehat{y}^{n_{0}}$ is controlled by $\eta \omega$. This means that for $\varepsilon$ small enough, $\mathfrak{I}_{f, y_{0}}(x)-\mathfrak{I}_{f, y_{0}}(\widehat{x})$ is controlled by $3^{p} \eta \omega$, if $\varepsilon$ is also chosen smaller than $\eta$. With Lemma 5 , this means that $\mathfrak{I}_{f, y_{0}}$ is continuous from $\mathcal{G}^{p}\left(\mathbb{R}^{n}\right)$ to $\mathcal{G}^{p}\left(\mathbb{R}^{m}\right)$. 
Remark 3. The previous proof is slightly different from the original proof of [32], where $f$ was required to be differentiable with a $\alpha$-Hölder continuous derivative to prove the existence of a solution.

\section{Integration of one-form along trajectories of finite $p$-variation with $2 \leqslant p<3$}

In this section, we consider the case of a path $x$ with finite $p$-variation, with $2 \leqslant p<3$.

\subsection{Second iterated integrals}

Let $x$ be a piecewise smooth function from $[0,1]$ to $\mathbb{R}^{d}$. For $i, j=1, \ldots, d$ and $(s, t) \in \Delta^{+}$, set

$$
\int_{s<r_{1}<r_{2}<t} \mathrm{~d} x_{r_{1}}^{i} \mathrm{~d} x_{r_{2}}^{j}=\int_{s}^{t}\left(x_{r_{1}}^{i}-x_{s}^{i}\right) \mathrm{d} x_{r_{1}}^{j}=\int_{s}^{t}\left(x_{r_{1}}^{i}-x_{s}^{i}\right)\left(x^{j}\right)_{r_{1}}^{\prime} \mathrm{d} r_{1} .
$$

Let $e_{1}, \ldots, e_{d}$ be the canonical basis of $\mathrm{V}=\mathbb{R}^{d}$, which implies that $x_{t}=$ $\sum_{i=1}^{d} e_{i} x_{t}^{i}$ for all $t \in[0,1]$. In order to simplify expressions, define $\int_{s}^{t} \mathrm{~d} x \otimes \mathrm{d} x$ as an element of $\mathrm{V} \otimes \mathrm{V}$ by

$$
\int_{s}^{t} \mathrm{~d} x \otimes \mathrm{d} x=\sum_{i, j=1}^{d} e_{i} \otimes e_{j} \int_{s<r_{1}<r_{2}<t} \mathrm{~d} x_{r_{1}}^{i} \mathrm{~d} x_{r_{2}}^{j} .
$$

Remark that for all $0 \leqslant s \leqslant u \leqslant t \leqslant 1$,

$$
\int_{s}^{t} \mathrm{~d} x \otimes \mathrm{d} x=\int_{s}^{u} \mathrm{~d} x \otimes \mathrm{d} x+\int_{u}^{t} \mathrm{~d} x \otimes \mathrm{d} x+\left(x_{u}-x_{s}\right) \otimes\left(x_{t}-x_{u}\right) .
$$

The space $\mathrm{V} \otimes \mathrm{V}$ is equipped with a norm $\|\cdot\|_{\mathrm{V} \otimes \mathrm{V}}$, also denoted by $|\cdot|$, such that $\|x \otimes y\|_{\mathrm{V} \otimes \mathrm{V}} \leqslant\|x\|_{\mathrm{V}}\|y\|_{\mathrm{V}}$.

\subsection{Estimating the error in the approximated Chasles' re- lation for an irregular control}

We assume still that $x$ is piecewise smooth. However, the only information we want to use is that $x$ is continuous, and the following assumptions on $x$ : there exists a function $\omega$ satisfying Assumption 1 and a real number $p \in[2,3)$ such that 
for all $(s, t) \in \Delta^{+}$,

$$
\begin{gathered}
\left|x_{t}-x_{s}\right|^{p} \leqslant \omega(s, t), \\
\left|\int_{s}^{t} \mathrm{~d} x \otimes \mathrm{d} x\right|^{p / 2} \leqslant \omega(s, t) .
\end{gathered}
$$

Assume also that $f: \mathbb{R}^{d} \mapsto \mathbb{R}^{d}$ is bounded, with a bounded derivative. This derivative is $\alpha$-Hölder continuous, with $\alpha$ such that $2+\alpha>p$.

Define the $p$-variation on $[s, t]$ of a function $y$ from $\Delta^{+}$to $\mathbb{R}^{d}$ by

$$
\operatorname{Var}_{p,[s, t]}(y)=\sup _{\substack{\Pi=\left\{t_{0}, \ldots, t_{k}\right\} \\ \text { partition of }[\mathrm{s}, \mathrm{t}]}}\left(\sum_{i=0}^{k-1}\left|y_{t_{i}, t_{i+1}}\right|^{p}\right)^{1 / p}
$$

When $y_{s, t}=y_{t}-y_{s}$, this definition is the same as the one of (2.7).

Under these conditions (3.2a) $-(3.2 \mathrm{~b})$,

$$
\operatorname{Var}_{p,[0,1]}(x)<+\infty \text { and } \operatorname{Var}_{p / 2,[0,1]}\left(\int_{s}^{t} \mathrm{~d} x \otimes \mathrm{d} x\right)<+\infty \text {. }
$$

However, by (3.1), this does not imply that the map $t \mapsto \int_{0}^{t} \mathrm{~d} x \otimes \mathrm{d} x$ has finite $p / 2$-variation.

Our goal is now to define a "good approximation" $y_{s, t}$ of $\int_{s}^{t} f\left(x_{r}\right) \mathrm{d} x_{r}$, so that this integral will be a limit of Riemann sums:

$$
\int_{s}^{t} f\left(x_{r}\right) \mathrm{d} x_{r}=\lim _{\delta \rightarrow 0} z_{s, t}^{\Pi^{\delta}} \text { with } z_{s, t}^{\Pi^{\delta}}=\sum_{i=0}^{k^{\delta}-1} y_{t_{i}^{\delta}, t_{i+1}^{\delta}}
$$

for a partition $\Pi^{\delta}=\left\{t_{0}^{\delta}, t_{1}^{\delta}, \ldots, t_{k^{\delta}}^{\delta}\right\}$ of $[s, t]$ whose mesh goes to zero with $\delta$. In the proof of Proposition 1, we have seen that one can consider the limit of $z_{s, t}^{\Pi^{\delta}}$, provided that he has a control of the form

$$
\left|y_{s, t}-y_{s, u}-y_{u, t}\right| \leqslant C \omega(s, t)^{\theta} \text { for all } 0 \leqslant s \leqslant u \leqslant t \leqslant 1
$$

for some constant $C$ and some $\theta>1$. The fact that $\theta>1$ is crucial, since the proof of Proposition 1 involves the Zeta function $\zeta(\theta)=\sum_{n \geqslant 1} 1 / n^{\theta}$.

In Lemma 1, we used the fact that $f$ is $\alpha$-Hölder continuous, that $\alpha>p$ and that $\left|x_{t}-x_{s}\right|^{p} \leqslant \omega(t, s)$. With only (3.2a) if $p \geqslant 2$, this no longer works even if $f$ has a bounded derivative, i.e., $\alpha=1$.

We are then forced to use a better estimate. If $x$ is smooth, then for $i=1, \ldots, d$,

$$
f^{i}\left(x_{t}\right)=f^{i}\left(x_{s}\right)+\sum_{i=1}^{d} \int_{s}^{t} \frac{\partial f^{i}}{\partial x_{j}}\left(x_{r}\right) \mathrm{d} x_{r}^{j}
$$


and then

$$
\int_{s}^{t} f^{i}\left(x_{r_{1}}\right) \mathrm{d} x_{r_{1}}^{i}=f^{i}\left(x_{s}\right) \int_{s}^{t} \mathrm{~d} x_{r_{1}}^{i}+\sum_{j=1}^{d} \int_{s}^{t} \int_{s}^{r_{1}} \frac{\partial f^{i}}{\partial x^{j}}\left(x_{r_{2}}\right) \mathrm{d} x_{r_{2}}^{j} \mathrm{~d} x_{r_{1}}^{i} .
$$

For any $y$ in $\mathrm{V}=\mathbb{R}^{d}$, denote by $\nabla f(y)$ be the bilinear form defined on $\mathrm{V} \otimes \mathrm{V}$ by

$$
\left\langle\nabla f(y), e_{i} \otimes e_{j}\right\rangle=\frac{\partial f^{j}}{\partial x_{i}}(y)
$$

A first approximation of the integral $\int_{s}^{t} f\left(x_{r}\right) \mathrm{d} x_{r}$ will be given by

$$
y_{s, t}=f\left(x_{s}\right)\left(x_{t}-x_{s}\right)+\nabla f\left(x_{s}\right) \int_{s}^{t} \mathrm{~d} x \otimes \mathrm{d} x .
$$

With (3.2a) and (3.2b), $\left|y_{s, t}\right|^{p} \leqslant N(f) \omega(0,1) \omega(s, t)$, where

$$
\begin{aligned}
& N(f)=\inf \left\{M \geqslant 0 \mid\|f\|_{\infty} \leqslant M,\|\nabla f\|_{\infty} \leqslant M\right. \leqslant \\
&\left.\sup _{x \neq y}|\nabla f(x)-\nabla f(y)| /|x-y|^{\alpha} \leqslant M\right\} .
\end{aligned}
$$

So, $y$ is of finite $p$-variation.

Lemma 5. For all $0 \leqslant s \leqslant u \leqslant t$,

$$
\left|y_{s, t}-y_{s, u}-y_{u, t}\right| \leqslant 2 N(f) \omega(s, t)^{\theta}
$$

with $\theta=(2+\alpha) / p>1$.

Proof. Let $a$ and $b$ be two points of $\mathbb{R}^{d}$. Then,

$$
\begin{aligned}
f^{i}(b) & =f^{i}(a)+\sum_{j=1}^{d} \int_{0}^{1} \frac{\partial f^{i}}{\partial x_{j}}(a+(b-a) r)\left(b^{j}-a^{j}\right) \mathrm{d} r \\
& =f^{i}(a)+\sum_{j=1}^{d} \frac{\partial f^{i}}{\partial x_{j}}(a)\left(b^{j}-a^{j}\right)+R^{i}(a, b)
\end{aligned}
$$

with

$$
\begin{aligned}
\left|R^{i}(a, b)\right| & =\left|\sum_{j=1}^{d} \int_{0}^{1}\left(\frac{\partial f^{i}}{\partial x_{j}}(a)-\frac{\partial f^{i}}{\partial x_{j}}(a+(b-a) r)\right)\left(b^{j}-a^{j}\right) \mathrm{d} r\right| \\
& \leqslant N(f)\|b-a\|^{1+\alpha}
\end{aligned}
$$

since the derivative of $f$ is $\alpha$-Hölder continuous (the quantity $N(f)$ has been defined in (3.4)). Set $R=\left(R^{1}, \ldots, R^{d}\right)$. 
Using (3.5) and (3.1),

$$
\left|y_{s, t}-y_{s, u}-y_{u, t}\right| \leqslant\left|\left(\nabla f\left(x_{u}\right)-\nabla f\left(x_{s}\right)\right) \int_{u}^{t} \mathrm{~d} x \otimes \mathrm{d} x\right|+\left|R\left(x_{u}, x_{s}\right)\left(x_{t}-x_{u}\right)\right| .
$$

As $\nabla f$ is $\alpha$-Hölder continuous, $x$ satisfies (3.2a) and $\int_{s}^{t} \mathrm{~d} x \otimes \mathrm{d} x$ satisfies (3.2b)

$$
\left|y_{s, t}-y_{s, u}-y_{u, t}\right| \leqslant C \omega(s, t)^{\theta} \text { with } \theta=\frac{2+\alpha}{p}>1
$$

The Lemma is then proved.

\subsection{Geometric multiplicative functionals}

One may re-use the proof of Proposition 1 exactly the same way with $y_{s, t}$ defined by (3.3), and not by (2.3). As we saw in the proof of Proposition 1 or in the proof of Lemma 5, the smoothness of $x$ plays no role.

However, if $x$ is not smooth, $\int_{s}^{t} \mathrm{~d} x \otimes \mathrm{d} x$ has to be defined. In fact, there is no general procedure to construct this term. However, for some particular $x$, such as the trajectories of some stochastic process, this is possible, but may be rather technical (see Sect. 12 for examples of stochastic processes for which the second order iterated integral has been defined). In the following, we assume that the second order iterated integral exists. But the path we consider is not $x$ but the couple $\left(x, \int \mathrm{d} x \otimes \mathrm{d} x\right)$, which no longer lives in $\mathbb{R}^{d}$, but in $\mathbb{R}^{d+d^{2}}$ and whose components satisfy some algebraic relations.

With this end in view, consider $\mathbf{x}_{s, t}=\left(\mathbf{x}_{s, t}^{1}, \mathbf{x}_{s, t}^{2}\right)$ defined for $(s, t) \in \Delta^{+}$, such that there exists a function $\omega$ satisfying Assumption 1 and a constant $p \in[2,3)$ for which:

$$
\begin{gathered}
\mathbf{x}_{s, t}^{1} \in \mathrm{V} \text { and } \mathbf{x}_{s, t}^{1}=x_{t}-x_{s}, \\
\left|\mathbf{x}_{s, t}^{1}\right|^{p} \leqslant \omega(s, t), \\
\mathbf{x}_{s, t}^{2} \in \mathrm{V} \otimes \mathrm{V} \text { and } \mathbf{x}_{s, t}^{2}=\mathbf{x}_{s, u}^{2}+\mathbf{x}_{u, t}^{2}+\mathbf{x}_{s, u}^{1} \otimes \mathbf{x}_{u, t}^{1}, \\
\left|\mathbf{x}_{s, t}^{2}\right|^{p / 2} \leqslant \omega(s, t)
\end{gathered}
$$

for all $0 \leqslant s \leqslant u \leqslant t \leqslant 1$. Such a $\mathbf{x}$ is called a multiplicative functional with $p$ variation controlled by $\omega$. Condition (3.7a) means that $\mathbf{x}^{1}$ may be identified with the path $x$. In this case, we say that $\mathbf{x}$ lies above $x$. Condition (3.7b) means that $x$ has finite $p$-variation, and is (3.2a). Condition (3.7c) is equivalent to (3.1), while $(3.7 \mathrm{~d})$ is analogue to $(3.2 \mathrm{~b})$.

When $x$ is piecewise smooth, set $\mathbf{x}_{s, t}^{1}=x_{t}-x_{s}$, and $\mathbf{x}_{s, t}^{2}=\int_{s}^{t} \mathrm{~d} x \otimes \mathrm{d} x$, and (3.7a)-(3.7d) are clearly satisfied. Denote by $\mathcal{S}^{2}(\mathrm{~V})$ the set of such multiplicative functionals. 
The distance $\delta_{p,[s, t]}$ is extended to $p \in[2,3)$ by

$$
\delta_{p,[s, t]}(\mathbf{x}, \mathbf{y})=\operatorname{Var}_{p,[s, t]}\left(\mathbf{x}^{1}-\mathbf{y}^{1}\right)+\operatorname{Var}_{p / 2,[s, t]}\left(\mathbf{x}^{2}-\mathbf{y}^{2}\right) .
$$

Denote still by $\mathcal{V}^{p}$ the topology it generates on the space of multiplicative functionals of finite $p$-variation.

We restrict ourself to multiplicative functionals which may be approximated by some elements in $\mathcal{S}^{2}(\mathrm{~V})$, where $\mathcal{S}^{2}(\mathrm{~V})$ is the set of multiplicative functionals $\mathbf{x}$ such that $\mathbf{x}_{s, t}^{1}=x_{t}-s_{s}$ and $\mathbf{x}_{s, t}^{2}=\int_{s}^{t} \mathrm{~d} x \otimes \mathrm{d} x$ for a piecewise smooth path $x$. Define $\mathcal{G}^{p}(\mathrm{~V})$ as the set a multiplicative functionals satisfying (3.7a)-(3.7d) for a given control $\omega$, and such that

$$
\mathbf{x} \text { may be approximated in } \mathcal{V}^{p} \text { by elements in } \mathcal{S}^{2}(\mathrm{~V})
$$

In this case, $\mathbf{x}$ is said to be a geometric multiplicative functional. In fact, (3.7e) is not really necessary in the case $2 \leqslant p<3$, but provides us with an intuitive view of integral driven by rough paths. This issue is discussed in Sect. 10.3 for Brownian motion.

Remark 4. If $\mathbf{x}$ belongs to $\mathcal{G}^{p}(\mathrm{~V})$, and $c=\left(c_{i, j}\right)_{i, j=1, \ldots, d}$ is an antisymmetric matrix, i.e., $c_{i, j}=-c_{j, i}$, seen as an element on $\mathrm{V} \otimes \mathrm{V}$, then the function $(s, t) \in \Delta^{+} \mapsto$ $\mathbf{x}_{s, t}+c(t-s)$ is also an element of $\mathcal{G}^{p}(\mathrm{~V})$. We give further explanations in Sects. 6.2 and 10.2 .

\subsection{Integration of a one-form}

In the previous sections, we have given all the elements to construct the integral of a differential one-form along a path $x$ of finite $p$-variations with $p \in[2,3)$, given that one also knows a geometric multiplicative $\mathbf{x}$ lying above $x$.

Once Lemma 5 has been proved, then one could use the same machinery as in the proof of Proposition 1, to prove that

$$
z_{s, t}=\lim _{\delta \rightarrow 0} \sum_{i=0}^{k^{\delta}-1} y_{t_{i}^{\delta}, t_{i+1}^{\delta}}
$$

exists and is unique for all partition $\left\{t_{0}^{\delta}, \ldots, t_{k^{\delta}}^{\delta}\right\}$ of $[s, t]$ when $y$ is given by (3.3).

Proposition 3 below summarizes this result. However, we will give in the next section a more complete construction of the integral of a one-form along the path $x$. In this new definition, the integral belongs to the set of geometric multiplicative functionals $\mathcal{G}^{p}(\mathrm{~W})$. This means that this integral could also be used as a path along which a another differential one-form is integrated. 
Proposition 3. Let $\mathbf{x}$ be an element in $\mathcal{G}^{p}(\mathrm{~V})$ lying above a continuous path $x$ for $p \in[2,3)$. Let $f$ be a continuous, bounded function with $\alpha$-Hölder continuous, bounded derivatives for $\alpha>p-2$. Then, for all $0 \leqslant s \leqslant t \leqslant 1$ and any family of partitions $\Pi^{\delta}=\left\{t_{0}^{\delta}, \ldots, t_{k^{\delta}}^{\delta}\right\}$ of $[s, t]$ whose meshes decrease to 0 as $\delta \rightarrow$ 0 , the limit $z_{s, t}$ defined in (3.8) exists and is unique, when $y_{s, t}=f\left(x_{s}\right) \mathbf{x}_{s, t}^{1}+$ $\nabla f\left(x_{s}\right) \mathbf{x}_{s, t}^{2}$. The limit $z_{s, t}$, which does not depend on the partitions $\Pi^{\delta}$, is denoted by $\int_{s}^{t} f\left(x_{r}\right) \mathrm{d} \mathbf{x}_{r}$, and is of finite p-variation. Finally, for all $0 \leqslant s \leqslant u \leqslant t \leqslant 1$, $z_{s, t}=z_{s, u}+z_{u, t}$.

We also have the equivalent of the continuity result of Proposition 2, assuming that $\mathbf{x}^{2}$ and $\widetilde{\mathbf{x}}^{2}$ are close enough in the sense given in Proposition 2, where $p$ is replaced by $p / 2$.

\subsection{The iterated integrals of $\int_{s}^{t} f\left(x_{r}\right) \mathrm{d} \mathbf{x}_{r}$}

Let us consider the differential form $f(x)=\sum_{i=1}^{d} f_{i}(x) \mathrm{d} x^{i}$, where the $f^{i}$ 's are functions from $\mathrm{V}=\mathbb{R}^{d}$ into $\mathrm{W}=\mathbb{R}^{m}$. The integral $\mathbf{z}_{s, t}^{1}=\int_{s}^{t} f\left(x_{r}\right) \mathrm{d} \mathbf{x}_{r}$ takes its value in $\mathrm{W}$, and is of finite $p$-variation. However, to construct the solution of a differential equation of the type

$$
y_{t}=y_{s}+\int_{s}^{t} f\left(y_{r}\right) \mathrm{d} x_{r}
$$

one may first define the integration of one-forms, and then use Picard's iteration principle. However, integrating with respect to a control of finite $p$-variation with $p \in[2,3)$ requires an element in $\mathcal{G}^{p}(\mathrm{~V})$. So, to use a fixed point theorem, we need to construct $\int_{s}^{t} f\left(\mathbf{x}_{r}\right) \mathrm{d} \mathbf{x}_{r}$ not only as an element of $\mathrm{W}$, but also as an element of $\mathcal{G}^{p}(\mathrm{~W})$.

To this end, set

$$
\begin{gathered}
\mathbf{y}_{s, t}^{1}=f\left(x_{s}\right) \mathbf{x}_{s, t}^{1}+\nabla f\left(x_{s}\right) \mathbf{x}_{s, t}^{2} \in \mathrm{W} \\
\mathbf{y}_{s, t}^{2}=f\left(x_{s}\right) \otimes f\left(x_{s}\right) \cdot \mathbf{x}_{s, t}^{2} \in \mathrm{W} \otimes \mathrm{W},
\end{gathered}
$$

and $\mathbf{y}_{s, t}=\left(\mathbf{y}_{s, t}^{1}, \mathbf{y}_{s, t}^{2}\right)$. In the definition of $\mathbf{y}^{2}$, we used a shorthand, which means in fact that

$$
\mathbf{y}_{s, t}^{2}=\sum_{i, j=1}^{d} f^{i}\left(x_{s}\right) \otimes f^{j}\left(x_{s}\right) \mathbf{x}_{s, t}^{2, i, j} .
$$

Denote by $\mathbf{z}_{s, t}^{1}$ the element of $\mathrm{W}$ given by $\int_{s}^{t} f\left(\mathbf{x}_{r}\right) \mathrm{d} \mathbf{x}_{r}$.

Let 1 denote an element of a one-dimensional space. We use the following computation rule: If $y$ belongs to $\mathrm{W}^{\otimes k}$ for some integer $k=1,2$, then $1 \otimes y=$ $y \otimes 1=y \in \mathrm{W}^{\otimes k}$. If $y$ and $z$ belong to $\mathrm{W}$, then $y \otimes z$ belongs to $\mathrm{W}^{\otimes 2}$. If $y$ belongs 
to $\mathrm{W}$ and $z$ belongs to $\mathrm{W}^{\otimes 2}$, then $y \otimes z=z \otimes y=0$. Set $T_{2}(\mathrm{~W})=1 \oplus \mathrm{W} \oplus \mathrm{W}^{\otimes 2}$. By the definition of the tensor product, if $x, y$ and $z$ belong to $T_{2}(\mathrm{~W})$, then for all $\alpha, \beta \in \mathbb{R},(\alpha x+\beta y) \otimes z=\alpha x \otimes z+\beta y \otimes z$ and $z \otimes(\alpha x+\beta y)=\alpha z \otimes x+\beta x \otimes y$.

Let $\Pi=\left\{t_{i} \mid t_{0} \leqslant \cdots \leqslant t_{\ell}\right\}$ be a partition of $[s, t]$. Set

$$
\mathbf{z}_{s, t}^{\Pi}=\left(1+\mathbf{z}_{t_{0}, t_{1}}^{1}+\mathbf{y}_{t_{0}, t_{1}}^{2}\right) \otimes \cdots \otimes\left(1+\mathbf{z}_{t_{\ell-1}, t_{\ell}}^{1}+\mathbf{y}_{t_{\ell-1}, t_{\ell}}^{2}\right) .
$$

The computation rules previously given mean that we keep only the elements in $T_{2}(\mathrm{~W})$, and not those in $\mathrm{W}^{\otimes k}$ for $k>2$. From Proposition [3, the projection $\mathbf{z}^{\Pi, 1}$ of $\mathbf{z}^{\Pi} \in T_{2}(\mathrm{~W})$ on $\mathrm{W}$ is equal to $\mathbf{z}_{s, t}^{1}$.

The proof that $\mathbf{z}^{\Pi}$ has a limit when the mesh of the partition $\Pi$ decreases to 0 is similar to the proof of Proposition 1. But we have also to estimate the "error" when $\mathbf{y}_{s, t}^{2}$ is split into $\mathbf{y}_{s, u}^{2}$ and $\mathbf{y}_{u, t}^{2}$.

Lemma 6. For all $0 \leqslant s \leqslant u \leqslant t \leqslant 1$, set

$$
\varepsilon(s, u, t)=\mathbf{y}_{s, t}^{2}-\mathbf{y}_{s, u}^{2}-\mathbf{y}_{u, t}^{2}-\mathbf{y}_{s, u}^{1} \otimes \mathbf{y}_{u, t}^{1} .
$$

There exists some constant $C$ depending only on $N(f), \omega(0,1)$ and $\alpha$ such that

$$
|\varepsilon(s, u, t)| \leqslant C \omega(s, t)^{\theta}
$$

with $\theta=(2+\alpha) / p>1$.

Proof. Recall that $x_{t}=\mathbf{x}_{0, t}^{1}$. Using (3.7c) and the relation

$$
\begin{aligned}
\mathbf{y}_{s, u}^{1} \otimes \mathbf{y}_{u, t}^{1} & =f\left(x_{s}\right) \mathbf{x}_{s, u}^{1} \otimes f\left(x_{u}\right) \mathbf{x}_{u, t}^{1} \\
& =f\left(x_{s}\right) \otimes f\left(x_{s}\right) \cdot \mathbf{x}_{s, u}^{1} \otimes \mathbf{x}_{u, t}^{1}+f\left(x_{s}\right) \otimes\left(f\left(x_{u}\right)-f\left(x_{s}\right)\right) \cdot \mathbf{x}_{s, u}^{1} \otimes \mathbf{x}_{u, t}^{1},
\end{aligned}
$$

we obtain that

$$
\begin{aligned}
\varepsilon(s, u, t)=\left(f\left(x_{s}\right) \otimes f\left(x_{s}\right)-f\left(x_{u}\right) \otimes\right. & \left.f\left(x_{u}\right)\right) \cdot \mathbf{x}_{s, t}^{2} \\
& -f\left(x_{s}\right) \otimes\left(f\left(x_{u}\right)-f\left(x_{s}\right)\right) \cdot \mathbf{x}_{s, u}^{1} \otimes \mathbf{x}_{u, t}^{1} .
\end{aligned}
$$

But

$$
\begin{aligned}
f\left(x_{s}\right) \otimes f\left(x_{s}\right)-f\left(x_{u}\right) & \otimes f\left(x_{u}\right) \\
& =\left(f\left(x_{s}\right)-f\left(x_{u}\right)\right) \otimes f\left(x_{s}\right)+f\left(x_{u}\right) \otimes\left(f\left(x_{s}\right)-f\left(x_{u}\right)\right) .
\end{aligned}
$$

Using the relation $f\left(x_{u}\right)-f\left(x_{s}\right)=\nabla f\left(x_{s}\right)\left(x_{u}-x_{s}\right)+R\left(x_{u}, x_{s}\right)$ together with (3.6), the boundedness of $f$ and $\nabla f$, we obtain that

$$
\begin{aligned}
\left|f\left(x_{s}\right)-f\left(x_{u}\right) \otimes f\left(x_{s}\right)\right| & \leqslant 2 N(f)^{2}\left(\left|x_{u}-x_{s}\right|+\left|x_{u}-x_{s}\right|^{1+\alpha}\right) \\
& \leqslant 2 N(f)^{2}\left(\omega(s, t)^{1 / p}+\omega(s, t)^{(1+\alpha) / p}\right) .
\end{aligned}
$$

Moreover, $\left|\mathbf{x}_{s, u}^{1} \otimes \mathbf{x}_{u, t}^{1}\right| \leqslant\left|\mathbf{x}_{s, u}^{1}\right| \cdot\left|\mathbf{x}_{u, t}^{1}\right| \leqslant \omega(s, t)^{2 / p}$. The Lemma is now easily proved by combining all the previous estimates. 
Proposition 4. As the mesh of $\Pi$ decreases to $0, \mathbf{z}_{s, t}^{\Pi}$ admits a limit, denoted by $\mathbf{z}_{s, t}$ and by $\int_{s}^{t} f\left(x_{r}\right) \mathrm{d} \mathbf{x}_{r}$. This limit is of finite p-variation.

Proof. Assume that $\Pi \cap(s, t)$ has more than one element. Let $t_{k}$ be an element of $\Pi \cap(s, t)$ such that $\omega\left(t_{k-1}, t_{k+1}\right) \leqslant 2 \omega(s, t) /|\Pi \cap(s, t)|$ (see Lemma 2). We use the convention that $t_{k-1}=s$ if $\Pi \cap\left(s, t_{k}\right)=\emptyset$, and that $t_{k+1}=t$ is $\Pi \cap\left(t_{k}, t\right)=\emptyset$. Using the computations' rules on $1 \oplus \mathrm{W} \oplus \mathrm{W}^{\otimes 2}$ provided in Sect. 3.5, one has

$$
\begin{aligned}
\left(1+\mathbf{z}_{t_{k-1}, t_{k}}^{1}+\right. & \left.\mathbf{y}_{t_{k-1}, t_{k}}^{2}\right) \otimes\left(1+\mathbf{z}_{t_{k}, t_{k+1}}^{1}+\mathbf{y}_{t_{k}, t_{k+1}}^{2}\right) \\
= & 1+\mathbf{z}_{t_{k-1}, t_{k+1}}^{1}+\mathbf{y}_{t_{k-1}, t_{k}}^{2}+\mathbf{y}_{t_{k}, t_{k+1}}^{2}+\mathbf{z}_{t_{k-1}, t_{k}}^{1} \otimes \mathbf{z}_{t_{k}, t_{k+1}}^{1} \\
= & 1+\mathbf{z}_{t_{k-1}, t_{k+1}}^{1}+\mathbf{y}_{t_{k-1}, t_{k+1}}^{2}-\varepsilon\left(t_{k-1}, t_{k}, t_{k+1}\right) \\
& +\mathbf{z}_{t_{k-1}, t_{k}}^{1} \otimes \mathbf{z}_{t_{k}, t_{k+1}}^{1}-\mathbf{y}_{t_{k-1}, t_{k}}^{1} \otimes \mathbf{y}_{t_{k}, t_{k+1}}^{1} .
\end{aligned}
$$

Set

$$
\begin{aligned}
\delta_{k} & =\mathbf{z}_{t_{k-1}, t_{k}}^{1} \otimes \mathbf{z}_{t_{k}, t_{k+1}}^{1}-\mathbf{y}_{t_{k-1}, t_{k}}^{1} \otimes \mathbf{y}_{t_{k}, t_{k+1}}^{1} \\
& =\left(\mathbf{z}_{t_{k-1}, t_{k}}^{1}-\mathbf{y}_{t_{k-1}, t_{k}}^{1}\right) \otimes \mathbf{z}_{t_{k}, t_{k+1}}^{1}-\mathbf{y}_{t_{k-1}, t_{k}}^{1} \otimes\left(\mathbf{y}_{t_{k}, t_{k+1}}^{1}-\mathbf{z}_{t_{k}, t_{k+1}}^{1}\right) .
\end{aligned}
$$

In Proposition 3, as in Proposition 1, for all $(r, u) \in \Delta^{+}$,

$$
\left|\mathbf{z}_{r, u}^{1}-\mathbf{y}_{r, u}^{1}\right| \leqslant K \omega(r, u)^{(2+\alpha) / p}
$$

for some constant $K$ depending on $f, \alpha$ and $p$. Besides, $\left|\mathbf{y}_{r, u}^{1}\right| \leqslant C \omega(r, u)^{1 / p}+$ $C \omega(r, u)^{2 / p}$, where $C$ is $\|f\|_{\infty}+\|\nabla f\|_{\infty}$. So, $\left|\delta_{k}\right| \leqslant C^{\prime} \omega(s, t)^{\theta^{\prime}}$, where the constants $\theta^{\prime}>1$ and $C^{\prime}$ depend only on $f, \alpha$ and $p$. Thus, if $\Pi^{\prime}=\Pi \backslash\left\{t_{k}\right\}$.

$$
\left|\mathbf{z}_{s, t}^{\Pi}-\mathbf{z}_{s, t}^{\Pi^{\prime}}\right| \leqslant\left|\varepsilon\left(t_{k-1}, t_{k}, t_{k+1}\right)\right|+\left|\delta_{k}\right| \leqslant C^{\prime \prime} \omega(s, t)^{\theta^{\prime \prime}},
$$

where the constants $\theta^{\prime \prime}>1$ and $C^{\prime \prime}$ depend only on $f, \alpha$ and $p$. The end of the proof is similar to the one of Proposition 1.

For the uniqueness of the limit, remark that if $\mathbf{z}$ and $\widetilde{\mathbf{z}}$ are two multiplicative functionals of finite $p$-variation such that $\mathbf{z}_{s, t}^{1}=\widetilde{\mathbf{z}}_{s, t}^{1}$ for all $(s, t) \in \Delta^{+}$, then

$$
\psi(s, t)=\mathbf{z}_{s, t}^{2}-\widetilde{\mathbf{z}}_{s, t}^{2}
$$

is additive, i.e., $\psi(s, u)+\psi(u, t)=\psi(s, t)$ for all $0 \leqslant s \leqslant u \leqslant t \leqslant 1$. Let $\mathbf{z}$ and $\widetilde{\mathbf{z}}$ be two cluster points of $\left(\mathbf{z}^{\Pi^{\delta}}\right)_{\delta>0}$ for a family $\left(\Pi^{\delta}\right)_{\delta>0}$ of partitions of $[0,1]$. By construction, $\mathbf{z}^{1}=\widetilde{\mathbf{z}}^{1}$. Moreover, for all integer $n \geqslant 1$,

$$
\left|\mathbf{z}_{s, t}^{2}-\widetilde{\mathbf{z}}_{s, t}^{2}\right| \leqslant \sum_{i=1}^{n-1}\left|\mathbf{z}_{t_{i}^{n}, t_{i+1}^{n}}^{2}-\widetilde{\mathbf{z}}_{t_{i}^{n}, t_{i+1}^{n}}^{2}\right| \leqslant 2 K \omega(s, t) \sup _{i=1, \ldots, n-1} \omega\left(t_{i}^{n}, t_{i+1}^{n}\right)^{\theta-1}
$$

where $t_{i}^{n}=s+i(t-s) / n$. Since $\omega$ is continuous near its diagonal, letting $n$ increase to infinity proves that $\mathbf{z}^{2}=\widetilde{\mathbf{z}}^{2}$, and the limit is unique. 
Corollary 2. The map

$$
\mathbf{x} \in \mathcal{G}^{p}(\mathrm{~V}) \longmapsto\left(\int_{s}^{t} f\left(x_{0}+\mathbf{x}_{0, r}^{1}\right) \mathrm{d} \mathbf{x}_{r} ;(s, t) \in \Delta^{+}\right) \in \mathcal{G}^{p}(\mathrm{~W})
$$

is continuous with respect to $\delta_{p}$.

It is because this map is continuous that, in view of (3.7e ), the integral belongs to $\mathcal{G}^{p}(\mathrm{~W})$.

The proof of Corollary 2 is similar to the one of Proposition 2 and Corollary 1 although a bit more complicated, since the number of terms to consider is more important.

\section{A faithful representation of paths}

We have explained in the previous sections how to construct solutions of differential equations controlled by path of finite $p$-variations with $p<3$. We have also constructed defined the integration along such irregular paths. We have seen that the "iterated integrals" appear naturally for defining our new objects. Our article is now devoted to provide the construction of these objects for all real number $p$.

In this section and the next one, we consider iterated integrals of piecewise smooth paths. We present some results, mainly due to K.-T. Chen (see [6] and related articles), which allows to perform some manipulations on smooth paths which could be expressed using algebraic computations. These results provides us with a very powerful tool. The first main result expresses that a piecewise smooth path $x$ can be uniquely defined by a power series involving its iterated integrals.

\subsection{The Chen series}

Let $x:[0, t] \rightarrow \mathrm{V}=\mathbb{R}^{d}$ be a piecewise smooth path. We shall assume that for all $s \in(0, t)$, there exists no $\varepsilon>0$ such that $x([s-\varepsilon, s])=x([s, s+\varepsilon])$. Such a path is called irreducible. Let $I=\left(i_{1}, \ldots, i_{k}\right)$ be a multi-index. Denote by $\int_{0}^{t} \mathrm{~d}_{I} x$ the iterated integral

$$
\int_{0}^{t} \mathrm{~d}_{I} x=\int_{0<s_{i_{1}}<\cdots<s_{i_{k}}<t} \mathrm{~d} x_{s_{1}}^{i_{1}} \cdots \mathrm{d} x_{s_{k}}^{i_{k}} .
$$

Introduce some indeterminates $X^{1}, \ldots, X^{d}$. Each of these indeterminates corresponds to a direction in the space $\mathbb{R}^{d}$. Thus, $X^{i}$ may be identified with the vector $e_{i}$ of the canonical basis of $\mathbb{R}^{d}$. For a multi-index $I=\left(i_{1}, \ldots, i_{k}\right)$, set $X^{I}=X^{i_{1}} \cdots X^{i_{k}}$. 
The non-commutative power series with indeterminates $X^{i}$ for $i=1, \ldots, d$ is given by

$$
\Phi([0, t], x)=\sum_{I \text { multi-index }} X^{I} \int_{0}^{t} \mathrm{~d}_{I} x
$$

provides a faithful representation of $x$, and $\Phi$ is called a Chen series.

Theorem 2 (K.-T. Chen). If $\Phi([0, t], x)=\Phi([0, t], y)$ for two paths $x$ and $y$, then $x=y$ on $[0, t]$ up to a translation.

In facts, manipulation on paths may be considered as manipulation on power series, as we will see.

\subsection{Concatenating two paths}

If $z$ denotes the path obtained by the concatenation of two paths $x:[0, t] \mapsto \mathbb{R}^{d}$ and $y:[t, s] \mapsto \mathbb{R}^{d}$ such that $x(t)=y(t)$, then

$$
\Phi([0, s], z)=\Phi([0, t], x) \Phi([t, s], y) .
$$

It means that the power series corresponding to $z$ is equal to the formal noncommutative product of the two power series corresponding to $x$ and $y$. Furthermore, if $\hat{x}(s)=x(t-s)$, then $\Phi([0, t], \hat{x})=\Phi([0, t], x)^{-1}$.

\subsection{Products of iterated integrals}

Another interesting property arises when one considers the product of two iterated integrals:

$$
\Psi_{s, t}=\left(\int_{s<u_{1}<\cdots<u_{j}<t} \mathrm{~d} x_{u_{1}}^{i_{1}} \ldots \mathrm{d} x_{u_{j}}^{i_{j}}\right)\left(\int_{s<u_{1}<\cdots<u_{j^{\prime}}<t} \mathrm{~d} x_{u_{1}}^{i_{1}^{\prime} \ldots \mathrm{d}} x_{u_{j^{\prime}}}^{i_{j^{\prime}}^{\prime}}\right) .
$$

If $I=\left(i_{1}, \ldots, i_{j}\right)$ and $I^{\prime}=\left(i_{1}^{\prime}, \ldots, i_{j^{\prime}}^{\prime}\right)$ are two multi-indices, denote by $I \in J$ the shuffle product of $I$ and $J$. Then

$$
\Psi_{s, t}=\sum_{K=\left(i_{1}^{\prime \prime}, \ldots, i_{j^{\prime \prime}}^{\prime \prime}\right)}\left(\int_{\text {multi-index } \in I \uplus J}\left(\int_{s<u_{1}<\cdots<u_{j^{\prime \prime}}<t} \mathrm{~d} x_{u_{1}}^{i_{1}^{\prime \prime}} \ldots \mathrm{d} x_{j_{j^{\prime \prime}}}^{i_{j^{\prime \prime}}^{\prime \prime}}\right) .\right.
$$

The shuffle product $I \mathbb{U} J$ of $I$ and $J$ is the set of all multi-indexes of length length $(I)+\operatorname{length}(J)$ such that for each $K$ in $I \cup J$, the elements of $K$ correspond either to the elements of $I$ or $J$, and $I$ (resp. $J$ ) is recovered if the elements of $I \uplus J$ belonging to $I$ (resp. $J$ ) are kept regardless the elements of $J$ (resp. $I$ ). 


\section{$5 \quad$ Lie algebra and enveloping algebra}

In this section, we continue to manipulate piecewise smooth paths and their Chen series. The main result of this section is Proposition $[$, which asserts that the Chen series $\Phi([s, t], x)$ could also be expressed as

$$
\Phi([s, t], x)=\exp \left(\sum_{I \text { multi-index }} \Theta_{I} \int_{s}^{t} \mathrm{~d}_{I} x\right),
$$

where the coefficients $\Theta_{I}$ belong to a particular subspace of the space containing the $X^{I}$ 's for all multi-index $I$.

\subsection{Enveloping algebra}

We present now some aspects of Lie algebras and enveloping algebras. The relation with $\Phi([0, t], x)$ is developed in Sect. 5.3. On this topic, see for example [40, Chap. 1].

Let $\mathcal{A}=\left\{a_{1}, \ldots, a_{n}\right\}$ be some letters. In Sect. 5.3, these letters will be identified with the indeterminates $X^{i}$. The letters may be used to construct some words $a_{i_{1}} \cdots a_{i_{k}}$ for some multi-index $I=\left(i_{1}, \ldots, i_{k}\right)$ of length $k$. The set of words with letters in $\mathcal{A}$ for which an empty word 1 is added is denoted by $\mathcal{A}^{*}$. Let $K$ be a ring containing $\mathbb{Q}$. A non-commutative polynomial $P$ is a linear combination over $K$ of words on $\mathcal{A}$ :

$$
P=\sum_{w \in \mathcal{A}^{*}} P_{w} w, P_{w} \in K \text { with only a finite number of terms. }
$$

The set of non-commutative polynomials is denoted by $K\langle\mathcal{A}\rangle$. Similarly, we may define formal series in the same way, but the number of terms in the sum is countable. The set of formal power series is denoted by $K\langle\langle\mathcal{A}\rangle\rangle$.

With $\mathcal{A}$ may be constructed a Lie algebra with bracket $[a, b]=a b-b a$ for all $a, b \in \mathcal{A}$. This Lie bracket may be extended to the set all non-commutative polynomials $P$. The set $K\langle\mathcal{A}\rangle$ is closed under $[\cdot, \cdot]$ and corresponds to the Lie algebra generated by $\mathcal{A}$.

Given this Lie algebra $\mathcal{L}$, it is known that there a unique associative algebra $\mathcal{E}(\mathcal{L})$ and a Lie algebra homomorphism $\varphi_{0}: \mathcal{L} \rightarrow \mathcal{E}(\mathcal{L})$ such that for all other associative algebra $\mathcal{B}$ and any Lie algebra homomorphism $\varphi$ (i.e., $[\varphi(a), \varphi(b)]=$ $\varphi([a, b])$ for all $a, b \in \mathcal{L})$ from $\mathcal{L}$ to $\mathcal{B}$, there exists a unique algebra homomorphism $f: \mathcal{E}(\mathcal{L}) \rightarrow \mathcal{B}$ such that $\varphi=f \circ \varphi_{0}$. The associative algebra $\mathcal{E}(\mathcal{L})$ is called the enveloping algebra. So, any algebra homomorphism from $\mathcal{L}$ into some associative algebra $\mathcal{B}$ may be extended to an algebra homomorphism from $\mathcal{E}(\mathcal{L})$ into $\mathcal{B}$.

Denote by $\mathcal{L}_{K}(\mathcal{A})$ the smallest submodule of $K\langle\mathcal{A}\rangle$ containing $\mathcal{A}$ and closed under the Lie bracket. 
Proposition 5. The algebra $K\langle\mathcal{A}\rangle$ is the enveloping algebra of $\mathcal{L}_{K}(\mathcal{A})$.

Given a Lie algebra $\mathcal{L}$, its enveloping algebra $\mathcal{E}(\mathcal{L})$ may be constructed by quotienting the tensor algebra

$$
\mathcal{T}(\mathcal{L})=\bigoplus_{n \geqslant 0} \mathcal{L}^{\otimes n}
$$

by the ideal $\mathcal{I}$ generated by the elements of the form $x \otimes y-y \otimes x-[x, y]$ for $x, y \in \mathcal{L}$.

\subsection{A characterization of the Lie polynomials}

An element of $\mathcal{L}_{K}(\mathcal{A})$ is called a Lie polynomial. A formal series $S$ that may be written $S=\sum_{n \geqslant 0} S_{n}$ where the $S_{n}$ 's are Lie polynomials is called a Lie series.

Remark 5. To understand the difference between a Lie polynomial and an element of $K\langle\mathcal{A}\rangle$, consider the following example: If the elements of a tangent space of a manifold are seen a first-order differential operators, then the Lie brackets $[x, y]$ of two of them remains a first-order differential operator and then belongs to the tangent space. If the letters $a_{i}$ are identified with vectors of a tangent space, a Lie polynomial belongs (formally) to the tangent space. Yet an element of the enveloping algebra is a general differential operator, with terms that could be of any order.

Let $\delta$ be the algebra homomorphism from $K\langle\langle\mathcal{A}\rangle\rangle \rightarrow K\langle\langle\mathcal{A}\rangle\rangle \times K\langle\langle\mathcal{A}\rangle\rangle$ defined by $\delta(a)=a \otimes 1+1 \otimes a$ for all $a \in \mathcal{A}$. The existence of $\delta$ as an algebra homomorphism is given by the property of the enveloping algebra.

Theorem 3. An element $P$ in $K\langle\langle\mathcal{A}\rangle\rangle$ is a Lie series if and only $\delta(S)=S \otimes 1+$ $1 \otimes S$. Furthermore, if $S$ is a Lie series, then the constant term $S_{1}$ is equal to 0 , where 1 is the empty word of $\mathcal{A}^{*}$.

From the condition on $\delta(P)$, the Lie polynomials are also called primitive.

For a formal series $S$ with zero constant term $\left(S_{1}=0\right)$, the exponential is defined to be

$$
\exp (S)=\sum_{n \geqslant 0} \frac{S^{n}}{n !}
$$

For a formal series of the form $S=1+T$ where $T$ have a zero constant term, the logarithm is defined to be

$$
\log (S)=\log (1+T)=\sum_{n \geqslant 1} \frac{(-1)^{n-1}}{n} T^{n} .
$$


Theorem 4. The following properties hold:

(i) Let $S$ be a series in $K\langle\langle\mathcal{A}\rangle\rangle$ with a constant term equal to 1. Then $\log (S)$ is a Lie series if and only if $\delta(S)=S \otimes S$.

(ii) The set of series in $K\langle\langle\mathcal{A}\rangle\rangle$ with a constant term equal to 1 such that $\log (S)$ is a Lie series is a group under multiplication.

An element in the group generated by $\exp (S)$ where $S$ is a Lie series with a constant term $S_{0}$ equal to 0 is called group-like. Theorems 3 and 4 assert that the map log is a bijection from group-like elements to primitive elements.

\subsection{The series of iterated integrals is a group-like element}

Now, take as alphabet $\mathcal{A}=\left(e_{1}, \ldots, e_{d}\right)$, where $e_{1}, \ldots, e_{d}$ is the canonical basis of $\mathrm{V}=\mathbb{R}^{d}$. The ring $K$ is $\mathbb{R}$. The product of two words $a$ and $b$ is replaced by the tensor product $a \otimes b$ of $a$ and $b$. Each of the $e_{i}$ 's corresponds to the indeterminates $X^{i}$, and this identification is used. That is,

$$
\Phi([s, t], x)=\sum_{\substack{I \text { multi-index } \\ I=\left(i_{1}, \ldots, i_{k}\right)}} e_{i_{1}} \otimes \cdots \otimes e_{i_{k}} \int_{s}^{t} \mathrm{~d}_{I} x .
$$

Thus, the series $\Phi([0, t], x)$, which belongs to $K\langle\langle\mathcal{A}\rangle\rangle$, may be seen as an element of $\mathbb{R} \oplus \mathrm{V} \oplus \mathrm{V}^{\otimes 2} \oplus \cdots$. More precisely, for all integer $k$, the element $\sum_{I=\left(i_{1}, \ldots, i_{k}\right)} e_{i_{1}} \otimes$ $\cdots \otimes e_{i_{k}} \int_{s}^{t} \mathrm{~d}_{I} x$ belongs to $\mathrm{V}^{\otimes k}$. In Sect. 4.2, we have seen that the concatenation of $x:[0, s] \mapsto \mathrm{V}$ and $x:[s, t] \mapsto \mathrm{V}$ creates a new paths $x:[0, t] \mapsto \mathrm{V}$ characterized by the series $\Phi([0, t], x)$ given by the product of $\Phi([0, s], x)$ and $\Phi([s, t], x)$. With our convention, (4.1) is rewritten $\Phi([0, t], x)=\Phi([0, s], x) \otimes \Phi([s, t], x)$.

Remark 6 (Another notation for the iterated integrals). If $e_{1}^{\prime}, \ldots, e_{d}^{\prime}$ is the canonical basis, identified with $e_{1}, \ldots, e_{d}$, of the dual $\mathrm{V}^{*}$ of $\mathrm{V}$, then define a multi-linear form $\int_{s}^{t} \mathrm{~d} x \otimes \cdots \otimes \mathrm{d} x$ on $\mathrm{V}^{*} \times \cdots \times \mathrm{V}^{*}$ by

$$
\left\langle\int_{s}^{t} \mathrm{~d} x \otimes \cdots \otimes \mathrm{d} x,\left(e_{i_{1}}^{\prime}, \ldots, e_{i_{k}}^{\prime}\right)\right\rangle=\int_{s<s_{1}<\cdots<s_{k}<t} \mathrm{~d} x_{s_{1}}^{i_{1}} \cdots \mathrm{d} x_{s_{k}}^{i_{k}} .
$$

Thus, $\int_{s}^{t} \mathrm{~d} x \otimes \cdots \otimes \mathrm{d} x$ is an element of the dual of $\mathrm{V}^{*} \times \cdots \times \mathrm{V}^{*}$, which is identified with $\mathrm{V}^{\otimes k}$, and $\int_{s}^{t} \mathrm{~d} x \otimes \cdots \otimes \mathrm{d} x$ is identified with

$$
\sum_{I=\left(i_{1}, \ldots, i_{k}\right) \in\{1, \ldots, d\}^{k}} e_{i_{1}} \otimes \cdots \otimes e_{i_{k}} \int_{s}^{t} \mathrm{~d}_{I} x .
$$

The following Proposition may be proved using the properties of the shuffle product and the Campbell-Hausdorff formula, and links the series constructed in Sect. 4 with our constructions of objects related to Lie algebras. 
Proposition $6([5])$. For any irreducible, piecewise smooth path $x:[0, t] \rightarrow \mathbb{R}^{d}$, $\Phi([0, t], x)$ is a group-like element when the elements $e_{i}$ of the basis of $\mathbb{R}^{d}$ are identified with the indeterminates $X^{i}$. Moreover,

$$
\log \Phi([0, t], x)=\sum_{I \text { multi-index }} \Theta_{I} \int_{s}^{t} \mathrm{~d}_{I} x
$$

where $\Theta_{I}$ belongs to $\mathcal{L}_{K}(\mathcal{A})=0 \oplus \mathrm{V} \oplus[\mathrm{V}, \mathrm{V}] \oplus[\mathrm{V},[\mathrm{V}, \mathrm{V}]] \oplus \cdots$ and does not involve more that length $(I)$ Lie brackets.

Remark 7 . There are very nice algebraic properties that can be considered on the series of type $\Phi([0, t], x)$. In particular, two structures of bi-algebra may be considered, one corresponding to concatenation of paths, the other one to product of the series and then using shuffle products (see for example [40, Sect. 1]). See also [47] for an example of use of the Poincaré-Birkhoff-Witt Theorem that gives a basis of Lie algebras.

\section{6 (Geometric) multiplicative functionals}

We have already encountered geometric multiplicative functionals in Sect. 3.3. In this section, we give a definition of geometric multiplicative functionals of any order.

Roughly speaking, a geometric multiplicative functional

$$
\mathbf{x}=\left(1, \mathbf{x}_{s, t}^{1}=x_{t}-x_{s}, \mathbf{x}_{s, t}^{2}, \ldots, \mathbf{x}_{s, t}^{k}\right)_{(s, t) \in \Delta^{+}}
$$

lying above a path $x$ corresponds to $x$ together with its first "iterated integrals", and such that the iterated integrals $\mathbf{x}^{\delta, \ell}=\int \mathrm{d} x^{\delta} \otimes \cdots \otimes \mathrm{d} x^{\delta}$ of piecewise smooth approximations $x^{\delta}$ of $x$ converge to $\mathbf{x}^{\ell}$ for $\ell=2, \ldots, k$.

If $x$ is a piecewise smooth path, we have seen that one can construct its Chen series $\Phi([0, t], x)$, which fully characterize $x$. Moreover, given a Chen series of a path $x$, one could formally solve a differential equation controlled by $x$ or consider the integral of a one-form along the path $x$, by writing these objects with the Chen series of $x$ (For example, see Sect. 6.3 below).

If $x$ is irregular, then knowing $x$ is not sufficient to define its iterated integrals (see Remark 4 and Sect.6.2 for example). However, when one knows a (geometric) multiplicative functional $\mathbf{x}=\left(1, \mathbf{x}^{1}, \mathbf{x}^{2}, \ldots, \mathbf{x}^{k}\right)$ lying above a path $x$ of finite $p$ variation with $k=\lfloor p\rfloor$, then it will be proved that there exists a procedure to extend $\mathbf{x}$ in a (geometric) multiplicative functional $\left(1, \mathbf{x}^{1}, \mathbf{x}^{2}, \ldots\right)$, and that this extension has some nice properties, especially with respect to the topology of generated by the norm of $p$-variation. Thus, one can construct an extension of the notion of Chen series for irregular paths, provided enough information is known 
on the path, i.e., its first "iterated integrals". And, in view of the results of Sects. 2 and 3, one will not be surprised by the results of Sects. 8, where integrals of oneform along irregular paths are constructed, and 9, where differential equations controlled by irregular paths are solved.

For any integer $k \geqslant 1$, set

$$
T_{k}(\mathrm{~V})=\mathbb{R} \oplus \mathrm{V} \oplus \mathrm{V}^{\otimes 2} \oplus \cdots \oplus \mathrm{V}^{\otimes k},
$$

which is a truncated tensor algebra. Let also $A_{k}(\mathrm{~V}) \subset T_{k}(\mathrm{~V})$ containing all the elements of $A(\mathrm{~V})=0 \oplus \mathrm{V} \oplus[\mathrm{V}, \mathrm{V}] \oplus[\mathrm{V},[\mathrm{V}, \mathrm{V}]] \oplus \ldots$, where all the terms involving more than $k$ Lie brackets are set to 0 . Similarly, computations on $T_{k}(\mathrm{~V})$ are done by setting to 0 all tensor products involving more than $k$ terms.

The norm we choose on $\mathrm{V}^{\otimes k}$ is such that

$$
\left\|x_{1} \otimes \cdots \otimes x_{k}\right\|_{\mathrm{V} \otimes k} \leqslant\left|x_{1}\right| \times \cdots \times\left|x_{k}\right| .
$$

This norm is also denoted by $|\cdot|$, and there are different possibilities for constructing such a norm (see for example [41]).

Definition 1. A multiplicative functional $\mathbf{x}=\left(\mathbf{x}^{0}, \mathbf{x}^{1}, \ldots, \mathbf{x}^{k}\right)$ of order $k$ is a function from $\Delta^{+}$into $T_{k}(\mathrm{~V})$ such that

$$
\begin{gathered}
\mathbf{x}: \Delta^{+} \rightarrow T_{k}(\mathrm{~V}) \text { is continuous, } \\
\mathbf{x}_{s, t}=\mathbf{x}_{s, u} \otimes \mathbf{x}_{u, t} \text { for all } 0 \leqslant s \leqslant u \leqslant t \leqslant 1 .
\end{gathered}
$$

Furthermore, $\mathbf{x}$ is said to be geometric if

$$
\log \mathbf{x}_{s, t} \in A_{k}(\mathrm{~V})
$$

and $\mathbf{x}^{0}=1$

Of course, in the previous definition, $\mathbf{x}^{i}$ denotes the projection of $\mathbf{x}$ on $\mathrm{V}^{\otimes i}$.

The set of multiplicative functionals with values in $T_{k}(\mathrm{~V})$ is denoted by $\mathcal{T}_{k}(\mathrm{~V})$. The subset of $\mathcal{T}_{\lfloor p\rfloor}(\mathrm{V})$ of of multiplicative functionals of finite $p$-variation is denoted by $\mathcal{M}^{p}(\mathrm{~V})$. Let us also denote by $\mathcal{G}^{p}(\mathrm{~V}) \subset \mathcal{M}^{p}(\mathrm{~V})$ the set of geometric multiplicative functionals of finite $p$-variation taking their values in $T_{\lfloor p\rfloor}(\mathrm{V})$.

Clearly, for a piecewise smooth path, $\mathbf{x}_{s, t}=\Phi([s, t], x)$ is a geometric multiplicative functional, and $\mathrm{x}_{s, t}^{i}=\int_{s}^{t} \mathrm{~d} x \otimes \cdots \otimes \mathrm{d} x$. Denote by $\mathcal{S}^{k}(\mathrm{~V})$ the set of geometric multiplicative functionals in $T_{k}(\mathrm{~V})$ lying above a piecewise smooth path given by the projection of $\Phi(x)$ on $T_{k}(\mathrm{~V})$.

Definition 1 extends the one given previously by (3.7a) $-(3.7 \mathrm{e})$, and (6.1c) replaces (3.7e). We will see below in Proposition 7 that these conditions are equivalent, provided that $\mathcal{M}^{p}(\mathrm{~V})$ is equipped with the good norm. 


\subsection{A norm on multiplicative functionals}

We use for multiplicative functionals in $\mathcal{T}_{k}(\mathrm{~V})$ the norm

$$
\|\mathbf{x}\|_{p,[s, t]}=\max _{i=1, \ldots, k}(\beta \Gamma(i / p))^{1 / p} \operatorname{Var}_{p / i,[s, t]}\left(\mathbf{x}^{i}\right)^{1 / i} .
$$

and $\|\mathbf{x}\|_{p}=\|\mathbf{x}\|_{p,[0,1]}$. Here $\beta$ is a positive constant, and $\Gamma$ is the Gamma function. Note that $\|\mathbf{x}\|_{q}$ is finite for all $q>p$ as soon as $\|\mathbf{x}\|_{p}$ is finite.

The space $\mathcal{T}_{k}(\mathrm{~V})$ equipped with the norm $\|\cdot\|_{p}$ is complete, but not separable.

Remark 8 . Note that any rough path $\mathbf{x}$ lies above the path $x$ defined by $x_{t}=\mathbf{x}_{0, t}$. For such a path, $x_{0}=0$, and $\operatorname{Var}_{p,[0,1]}$ is a norm on this space of functions from $x:[0,1] \rightarrow \mathrm{V}$ with $x_{0}=0$, and not only a semi-norm.

Definition 2. We say that $\mathbf{x}$ in $\mathcal{T}_{k}(\mathrm{~V})$ is of finite p-variation controlled by $\omega$ : $\Delta^{+} \rightarrow \mathbb{R}_{+}$(satisfying Assumption [1) when $\|\mathbf{x}\|_{p,[s, t]}^{p} \leqslant \omega(s, t)$ for all $(s, t) \in \Delta^{+}$.

Lemma 7. A multiplicative functional $\mathbf{x}$ in $\mathcal{T}_{k}(\mathrm{~V})$ is of finite p-variation controlled by $\omega$ if and only if

$$
\left|\mathbf{x}_{s, t}^{i}\right| \leqslant \frac{\omega(s, t)^{i / p}}{\beta \Gamma(i / p)} \text { for } i=1, \ldots, k .
$$

Moreover, Lemma 3 also holds for multiplicative functionals: If $\left(\mathbf{x}^{n}\right)_{n \in \mathbb{N}}$ is a sequence of multiplicative functionals converging to $\mathbf{x}$ in $\|\cdot\|_{p}$, then there exists an $\omega: \Delta^{+} \rightarrow \mathbb{R}_{+}$satisfying Assumption 1 such that $\mathbf{x}^{n}$ (or possibly only for the element of a subsequence of $\left.\left(\mathbf{x}^{n}\right)_{n \in \mathbb{N}}\right)$ and $\mathbf{x}$ are controlled by $\omega$, and for all $\varepsilon>0$, there exists some $n$ such that for all $m \geqslant n$,

$$
\left|\mathbf{x}_{s, t}^{m, i}-\mathbf{x}_{s, t}^{i}\right| \leqslant \varepsilon \frac{\omega(s, t)^{i / p}}{\beta \Gamma(i / p)} .
$$

Let $x$ be a smooth path, and $\mathrm{x}$ be its associated geometric multiplicative functional, i.e., $\mathbf{x}_{s, t}^{i}=\int_{s}^{t} \mathrm{~d} x \otimes \cdots \otimes \mathrm{d} x$. Hence, there exists a constant $C$ such that $\left|\mathbf{x}_{s, t}^{i}\right| \leqslant C(t-s)^{i} / i$ !. Thus, the choice of the norm $\|\cdot\|_{p}$ is coherent with that fact that a smooth function is controlled by $\omega(s, t)=C|t-s|$.

We said earlier that (6.1c) replaces (3.7e). In fact, the two hypotheses may be seen as equivalent.

Proposition 7 ([32, Lemma 2.3.1, p. 259]). The space $\mathcal{G}^{p}(\mathrm{~V})$ is the closure of $\mathcal{S}^{\lfloor p\rfloor}(\mathrm{V})$ (i.e., geometric multiplicative functionals lying above piecewise smooth trajectories) with respect to $\|\cdot\|_{p}$.

Practically, geometric multiplicative functionals will be constructed by approximating irregular trajectories by piecewise linear functions whose iterated integrals converge. This is why (3.7e) is generally more useful than (6.1c). 
Lemma 8 (A convergence and compactness result). Let $p$ be a fixed real number. Let $\left(\mathrm{x}^{n}\right)_{n \in \mathbb{N}}$ be a family of multiplicative functionals in $\mathcal{T}_{k}(\mathrm{~V})$ such that for some $q<p$ and for $i=1, \ldots, k$,

$$
\begin{gathered}
\forall \varepsilon>0, \exists \eta>0 \text { such that }|s-t|<\eta \Longrightarrow\left|\mathbf{x}_{s, t}^{n, i}\right|<\varepsilon, \\
\sup _{n \in \mathbb{N}} \operatorname{Var}_{q / i,[0,1]}\left(\mathbf{x}^{n, i}\right)<+\infty .
\end{gathered}
$$

Then there exist a subsequence $\left(n_{\ell}\right)_{\ell \in \mathbb{N}}$ and a multiplicative functional $\mathbf{x}$ in $\mathcal{T}_{k}(\mathrm{~V})$ such that

$$
\left\|\mathbf{x}^{n_{\ell}}-\mathbf{x}\right\|_{p} \underset{\ell \rightarrow \infty}{\longrightarrow} 0
$$

In other words, $\left(\mathbf{x}^{n}\right)_{n \in \mathbb{N}}$ is relatively compact in $\left(\mathcal{T}_{k}(\mathrm{~V}),\|\cdot\|_{p}\right)$.

Moreover, if $\mathbf{x}^{n}$ lies above a path $x^{n}$ and $\left(x_{0}^{n}\right)_{n \in \mathbb{N}}$ is relatively compact in $\mathbb{R}$, then any possible $\mathbf{x}$ limit of $\left(\mathbf{x}^{n}\right)_{n \in \mathbb{N}}$ lies above a path $x$, which is also a possible limit of the sequence $\left(x^{n}\right)_{n \in \mathbb{N}}$ in the space of continuous functions.

Remark 9. In particular, (6.4a) and (6.4b) are true if $\left(\mathbf{x}_{0}^{n}\right)_{n \in \mathbb{N}}$ is bounded and there exists a function $\omega$ satisfying Assumption 1 such that $\left\|\mathbf{x}^{n}\right\|_{q,[s, t]} \leqslant \omega(s, t)$ for all $(s, t) \in \Delta^{+}$and any $n \in \mathbb{N}$.

Remark 10. Thanks to the Ascoli theorem and the relation (6.1b), the condition (6.4a) is also equivalent to saying that the sequences of functions $\left(t \mapsto \mathbf{x}_{0, t}^{n, i}\right)_{n \in \mathbb{N}}$ are relatively compact in the space of continuous functions for $i=1, \ldots, k$. This is a fact we use in the proof of this Lemma.

Proof. If $\mathbf{y}$ is a multiplicative functional in $\mathcal{T}_{k}(\mathrm{~V}),(\underline{6.1 \mathrm{~b}})$ implies that for all $(s, t) \in$ $\Delta^{+}$and $i=1, \ldots, k$,

$$
\mathbf{y}_{0, t}^{i}=\mathbf{y}_{0, s}^{i}+\sum_{k+\ell=i, \ell \geqslant 1} \mathbf{y}_{0, s}^{k} \otimes \mathbf{y}_{s, t}^{\ell}
$$

Hence,

$$
\begin{aligned}
\left|\mathbf{y}_{0, t}^{i}-\mathbf{y}_{0, s}^{i}\right| & \leqslant \sum_{k+\ell=i, \ell \geqslant 1}\left|\mathbf{y}_{0, s}^{k}\right| \cdot\left|\mathbf{y}_{s, t}^{\ell}\right| \\
& \leqslant C\|\mathbf{y}\|_{\infty} \sum_{\ell=1}^{i}\left|\mathbf{y}_{s, t}^{\ell}\right| \leqslant C^{2}\|\mathbf{y}\|_{\infty}\|\mathbf{y}\|_{q,[s, t]} .
\end{aligned}
$$

where $C$ depends only on $q$ and $i$.

With (6.4a) and (6.5), it is clear that $\left(t \mapsto \mathbf{x}_{0, t}^{n, i}\right)_{n \in \mathbb{N}}$ is bounded and equicontinuous for $i=1, \ldots, k$. Thus, Ascoli's Theorem implies that there exists a continuous function $t \mapsto \mathbf{x}_{0, t} \in \mathbb{R}^{d+d^{2}+\cdots+d^{k}}$ such that, at least along a subsequence, $t \mapsto \mathbf{x}_{0, t}^{n}$ converges uniformly to $t \mapsto \mathbf{x}_{0, t}$. 
Using (6.5) recursively, one can construct from $t \mapsto \mathbf{x}_{0, t}$ a function $(s, t) \in$ $\Delta^{+} \mapsto \mathbf{x}_{s, t}$ that is a multiplicative functional and such that, at least along a subsequence, $\mathbf{x}_{s, t}^{n} \underset{n \rightarrow \infty}{\longrightarrow} \mathbf{x}_{s, t}$ uniformly in $s$ and $t$.

It is now easily seen that $\operatorname{Var}_{p / i}\left(\mathbf{x}^{i}\right) \leqslant \liminf \inf _{n \rightarrow \infty} \operatorname{Var}_{p / i}\left(\mathbf{x}^{n, i}\right)$ for $i=1, \ldots, k$. Thus, $\mathbf{x}$ is of finite $p$-variation. With a variation of (2.8), one obtains that for all $p>q,\left\|\mathbf{x}-\mathbf{x}^{n}\right\|_{p,[s, t]}$ goes to 0 , at least along a subsequence.

The second part of the Lemma is clear from Ascoli's Theorem.

Corollary 3 (A tightness result). Let $\left(\mathbf{X}^{n}\right)_{n \in \mathbb{N}}$ be a family of random variables taking their values in $\mathcal{T}_{k}(\mathrm{~V})$. Assume that the family of stochastic processes $\left(\mathbf{X}_{0, t}^{n} ; t \in\right.$ $[0,1])_{n \in \mathbb{N}}$ is tight in the space of continuous functions with the uniform norm, and that for all $\varepsilon>0$, there exists some constant $C$ large enough so that

$$
\sup _{n \in \mathbb{N}} \mathbb{P}\left[\left\|\mathbf{X}^{n}\right\|_{q}>C\right]<\varepsilon
$$

for some real number $q \geqslant 1$. Then $(\mathbf{X})_{n}$ is tight in $\left(\mathcal{I}_{k}(\mathrm{~V}),\|\cdot\|_{p}\right)$ (hence in $\mathcal{M}^{p}(\mathrm{~V})$ if $k=\lfloor p\rfloor)$ for all $p>q$.

Remark 11. Since $\left(\mathcal{T}_{k}(\mathrm{~V}),\|\cdot\|_{p}\right)$ is not separable, a sequence $\left(\mathbf{X}^{n}\right)_{n \in \mathbb{N}}$ may be tight in this space but fails to satisfy (6.6).

Remark 12. Owing to (6.1b) , the tightness of $\left(t \mapsto \mathbf{X}_{0, t}^{n, i}\right)_{n \in \mathbb{N}}$ for all $i \in\{1, \ldots, k\}$ is equivalent to saying that for all $\varepsilon>0$ and any $C>0$, there exists some $\eta>0$ small enough such that

$$
\sup _{n \in \mathbb{N}} \sup _{i=1, \ldots, k} \mathbb{P}\left[\sup _{|t-s|<\eta}\left|\mathbf{X}_{s, t}^{i, n}\right|>C\right] \leqslant \varepsilon .
$$

Proof. The proof is immediate from Lemma 8 and Remark 12, since the subsets $K$ of $\mathcal{T}_{k}(\mathrm{~V})$ of the form $K_{0} \cap K_{1}$ for a given $C>0$ are relatively compact in $\left(\mathcal{T}_{k}(\mathrm{~V}),\|\cdot\|_{p}\right)$, where the sets of functions $\left(t \mapsto \mathbf{x}_{0, t}^{i}\right)_{\mathbf{x} \in K_{0}}$ are equi-continuous for $i=$ $1, \ldots, k$ and $K_{1}$ contains the multiplicative functionals such that $\sup _{\mathbf{x} \in K_{1}}\|\mathbf{x}\|_{q}<C$ for some $q<p$ and a given constant $C$.

\subsection{Back to the case $2 \leqslant p<3$}

To provide a better understanding of what a geometric multiplicative functional could be, consider a multiplicative functional $\mathbf{x}$ in $\mathcal{T}_{2}(\mathrm{~V})$ with $\mathrm{V}=\mathbb{R}^{d}$. Set

$$
\mathfrak{S}_{s, t}(\mathbf{x})=\frac{1}{2} \mathbf{x}_{s, t}^{1} \otimes \mathbf{x}_{s, t}^{1} \text { and } \mathfrak{A}_{s, t}(\mathbf{x})=\mathbf{x}_{s, t}^{2}-\mathfrak{S}_{s, t}(\mathbf{x}) .
$$

Thus, $\mathfrak{A}(\mathbf{x})=\left(\mathfrak{A}^{i, j}(\mathbf{x})\right)_{i, j=1, \ldots, d}$ with $\mathfrak{A}_{s, t}^{i, j}(\mathbf{x})=\frac{1}{2}\left(\mathbf{x}_{s, t}^{2, i, j}-\mathbf{x}_{s, t}^{2, j, i}\right)$. Remark that $\mathfrak{S}(\mathbf{x})$ and $\mathfrak{A}(\mathbf{x})$ are respectively the symmetric part and the antisymmetric part of $\mathbf{x}^{2}$. 
Moreover, $\mathfrak{S}(\mathbf{x})$ depends only on $\mathbf{x}^{1}$, and if $\mathbf{x}^{1}$ lies above a path $x$, then $\mathfrak{S}(\mathbf{x})=$ $\mathfrak{S}(x)$. Moreover, if $x$ is of finite $p$-variation, the map $x \mapsto \mathfrak{S}(x)$ is continuous for the topology generated by $\operatorname{Var}_{p / 2,[s, t]}(\cdot)+\|\cdot\|_{\infty}$.

On the other side, if $\mathbf{x}$ is a natural geometric multiplicative functional in $\mathcal{S}^{2}(\mathrm{~V})$ lying above a smooth path $x$, then $\mathfrak{A}_{s, t}^{i, j}(\mathbf{x})$ is the area contained between the curve $\left(x_{r}^{i}, x_{r}^{j}\right)_{r \in[s, t]}$ and the chord $\overline{\left(x_{s}^{i}, x_{s}^{j}\right)\left(x_{t}^{i}, x_{t}^{j}\right)}$. Denote also this area by $\mathfrak{A}_{s, t}^{i, j}(x)$.

Lemma 9. For all $0 \leqslant s \leqslant t \leqslant 1$, the map $x \in \mathcal{S}^{2}\left(\mathbb{R}^{d}\right) \mapsto \mathfrak{A}_{s, t}(x)$ is not continuous with respect to the uniform norm (except if $d=1$, in which case $\left.\mathfrak{A}_{s, t}(x)=0\right)$.

Proof. Assume that $d=2$ and identify $\mathbb{R}^{2}$ with the complex plane $\mathbb{C}$. Set $x_{t}^{n}=$ $n^{-1} \mathrm{e}^{\mathrm{i} n^{2} t}$. Then, $\mathfrak{A}_{0,2 \pi}\left(x^{n}\right)=\pi$, but $x^{n}$ converges uniformly to 0 .

Thus, to construct a geometric multiplicative functional $\mathbf{x}$ lying above $x$ of finite $p$-variation with $p \in[2,3)$, one has to focus only on the construction of the antisymmetric part $\mathfrak{A}(\mathbf{x})$ of $\mathbf{x}$. This also provides us a with nice geometric interpretation. However, note that this choice is not unique.

Lemma 10. If $\mathbf{x}$ is in $\mathcal{G}^{p}(\mathrm{~V})$ for $p \in[2,3)$, and $\varphi=\left(\varphi_{i, j}\right)_{i, j=1, \ldots, d}$ is a function from $[0,1]$ to $d \times d$-antisymmetric matrices, and of finite $p / 2$-variation. Then $\widehat{\mathbf{x}}$ defined by

$$
\widehat{\mathbf{x}}_{s, t}^{1}=\mathbf{x}_{s, t}^{1} \text { and } \widehat{\mathbf{x}}_{s, t}^{2, i, j}=\mathbf{x}_{s, t}^{2, i, j}+\varphi_{i, j}(t)-\varphi_{i, j}(s) .
$$

Then $\widehat{\mathbf{x}}$ is also in $\mathcal{G}^{p}(\mathrm{~V})$.

Proof. If $\mathbf{x}$ is a geometric multiplicative functional, and $e_{1}, \ldots, e_{d}$ is the canonical basis of $\mathrm{V}=\mathbb{R}^{d}$, then rewrite $\mathbf{x}_{s, t}$ as

$$
\mathbf{x}_{s, t}=1+\sum_{i=1}^{d} e_{i} \mathbf{x}_{s, t}^{1, i}+\sum_{i, j=1}^{d} e_{i} \otimes e_{j} \mathbf{x}_{s, t}^{2, i, j}
$$

The quantity $\log \left(\mathbf{x}_{s, t}\right)$, which belongs to $A_{2}(\mathrm{~V})$ (see (‥1c) ), may be explicitly computed:

$$
\log \left(\mathbf{x}_{s, t}\right)=\sum_{i=1}^{d} e_{i} \mathbf{x}_{s, t}^{1, i}+\frac{1}{2} \sum_{i, j=1}^{d}\left[e_{i}, e_{j}\right] \mathfrak{A}_{s, t}^{i, j}(\mathbf{x}),
$$

where $\left[e_{i}, e_{j}\right]=e_{i} \otimes e_{j}-e_{j} \otimes e_{i}$ is the Lie bracket of $e_{i}$ and $e_{j}$. It is clear that $\widehat{\mathbf{x}}$ is a multiplicative functional in $\mathcal{M}^{p}(\mathrm{~V})$, and as $\varphi$ is antisymmetric, i.e., $\varphi_{i, j}(t)=$ $-\varphi_{j, i}(t)$ for all $t \in[0,1]$,

$$
\log \left(\widehat{\mathbf{x}}_{s, t}\right)=\log \left(\mathbf{x}_{s, t}\right)+\frac{1}{2} \sum_{i, j=1}^{d}\left[e_{i}, e_{j}\right]\left(\varphi_{i, j}(t)-\varphi_{i, j}(s)\right) .
$$

Thus, $\log \left(\widehat{\mathbf{x}}_{s, t}\right)$ belongs to $A_{2}(\mathrm{~V})$ for all $(s, t) \in \Delta^{+}$. Then, $\widehat{\mathbf{x}}$ belongs to $\mathcal{G}^{p}(\mathrm{~V})$. 
We have then a way to construct as many geometric multiplicative functionals lying above a path $x$ as we want. The difference between the geometric multiplicative functionals $\mathbf{y}=\int f\left(x_{s}\right) \mathrm{d} \mathbf{x}_{s}$ and $\widehat{\mathbf{y}}=\int f\left(x_{s}\right) \mathrm{d} \widehat{\mathbf{x}}_{s}$ is immediate in view of (3.9a) $-(3.9 \mathrm{~b})$ :

$$
\begin{gathered}
\widehat{\mathbf{y}}_{s, t}^{1}=\mathbf{y}_{s, t}^{1}+\sum_{i, j=1}^{d} \int_{s}^{t} \frac{\partial f_{i}}{\partial x_{j}}\left(x_{r}\right) \mathrm{d} \psi_{j, i}(r) \\
\text { and } \widehat{\mathbf{y}}_{s, t}^{2}=\mathbf{y}_{s, t}^{2}+\int_{s}^{t} f\left(x_{r}\right) \otimes f\left(x_{r}\right) \cdot \mathrm{d} \psi(r) .
\end{gathered}
$$

In Sect. 10.2 below, these results will be used to compare the theory of integration given by this theory and the Stratonovich integral for Brownian motion (see especially (10.2) and (10.3) $)$.

The fact that $\varphi$ was taken additive (i.e., $\varphi(s, u)+\varphi(u, s)=\varphi(s, t)$ for all $0 \leqslant$ $s \leqslant u \leqslant t \leqslant 1)$ in Lemma 10 is justified by the following Lemma.

Lemma 11 ([32, Lemma 2.2.3, p. 250]). If $\mathbf{x}$ and $\widehat{\mathbf{x}}$ are two multiplicative functionals in $\mathcal{M}^{p}(\mathrm{~V})$ which agree for all order smaller than $k=\lfloor p\rfloor$ (i.e., $\quad \mathbf{x}_{s, t}^{i}=\widehat{\mathbf{x}}_{s, t}^{i}$ for $i=0,1, \ldots, k-1)$, then $\varphi(s, t)=\mathbf{x}_{s, t}^{k}-\widehat{\mathbf{x}}_{s, t}^{k}$ is additive, and is of finite $p / k$-variation.

\subsection{Intermezzo: solving linear differential equations}

It is now time to justify the usefulness of geometric multiplicative functionals, and the choice of the semi-norm $\|\cdot\|_{p,[s, t]}$.

Assume that $x$ is a piecewise smooth path, and that $\mathbf{x}$ is its Chen series (in $\mathcal{T}_{\infty}(\mathrm{V})$, i.e., consider all its iterated integrals).

Let $C$ be a $m \times m$-matrix and assume to begin with that $d=1$. Then it is well known that the solution of the differential equation $\mathrm{d} y_{t}=C y_{t} \mathrm{~d} x_{t}$ is $y_{t}=$ $\exp \left(C x_{t}\right) y_{0}$, where exp is the exponential of matrices.

Now, consider a family $C_{1}, \ldots, C_{d}$ of $m \times m$-matrices, and the differential equation

$$
\mathrm{d} y_{t}=\sum_{i=1}^{d} C_{i} y_{t} \mathrm{~d} x_{t}^{i}
$$

As $y$ appears in the right-hand side of (6.7), one may replace it by its value given by (6.7). Thus,

$$
y_{t}=y_{s}+\sum_{i=1}^{d} C_{i} y_{s} \int_{s}^{t} \mathrm{~d} x_{s}^{i}+\sum_{i, j=1}^{d} \int_{s<r_{1}<r_{2}<t} C_{i} C_{j} y_{s} \mathrm{~d} x_{r_{1}}^{i} \mathrm{~d} x_{r_{2}}^{j} .
$$

For a multi-index $I=\left(i_{1}, \ldots, i_{k}\right)$, set $C_{I}=C_{i_{1}} C_{i_{2}} \cdots C_{i_{k}}$. 
Re-iterating the substitution, one obtains the formal power series

$$
y_{t}=\left(\mathrm{Id}+\sum_{I \text { multi-index }} C_{I} \int_{s}^{t} \mathrm{~d}_{I} x\right) y_{s} .
$$

As the $C_{i}$ 's appear at the same place as the indeterminates $X^{i}$ in the formal Chen's series $\Phi([s, t], x)$ of $x,(\underline{6.8})$ may also be written, using (5.1),

$$
y_{t}=\exp \left(\sum_{I \text { multi-index }} \Theta_{I}\left(C_{1}, \ldots, C_{d}\right) \int_{s}^{t} \mathrm{~d}_{I} x\right) y_{s},
$$

where $\Theta_{I}\left(C_{1}, \ldots, C_{d}\right)$ is a linear combination of terms in the smallest space of matrices containing $\left\{C_{1}, \ldots, C_{d}\right\}$ and closed under the Lie brackets $[A, B]=A B-$ $B A$.

Of course, one may wonder if the series

$$
\Xi(x)=\mathrm{Id}+\sum_{I \text { multi-index }} C_{I} \int_{s}^{t} \mathrm{~d}_{I} x
$$

converges. But there are $i^{d}$ multi-indexes $I$ of length $i$, and $\left\|C_{I}\right\| \leqslant c^{i}=\left(\sup _{i=1, \ldots, d}\|C\|\right)^{i}$. As $\left|\int_{s}^{t} \mathrm{~d}_{I} x\right| \leqslant C|t-s|^{i} / i$ ! for some constant $C$ which depends on the bounds of the derivatives of $x$, then

$$
\|\Xi(x)\| \leqslant \sum_{i=0}^{+\infty} \frac{d^{i} c^{i}|t-s|^{i}}{i !}<+\infty .
$$

Thus, using the condition that a rough path $\mathbf{x}$ is of finite $p$-variation controlled by $\omega$, one may construct the solution $y$ of

$$
\mathrm{d} y_{t}=\sum_{i=1}^{d} C_{i} y_{t} \mathrm{~d} \mathbf{x}_{s, t}^{1, i}, \text { for } t \geqslant s
$$

by setting

$$
y_{t}=\left(\mathrm{Id}+\sum_{I \text { multi-index }} C_{I} \mathbf{x}_{s, t}^{\text {length } I, I}\right) y_{s} .
$$

Inequality (6.2) implies that the series that appears in this expression is convergent, in the sense of the norm of operators.

\subsection{Extending multiplicative functionals to any order}

We have defined geometric multiplicative functionals as elements of the "truncated" tensor algebra $T_{k}(\mathrm{~V})$, while an expression like (6.10) requires to know a geometric multiplicative functionals in the tensor algebra $\mathcal{T}_{\infty}(\mathrm{V})$. Does one need to know all the terms of a geometric multiplicative functional? 
Theorem 5 ([32, Theorem 2.2.1]). Let $\mathbf{x}$ be a multiplicative functional in $\mathcal{T}_{k}(\mathrm{~V})$, where $k=\lfloor p\rfloor$ for some $p \geqslant 1$. Assume that there exists a function $\omega$ satisfying Assumption 1 such that for all $(s, t) \in \Delta^{+}$,

$$
\left|\mathbf{x}_{s, t}^{i}\right| \leqslant \frac{\omega(s, t)^{i / p}}{\beta \Gamma(i / p)}
$$

for $i=1, \ldots, k$. Then, if $\beta$ is large enough (however, the choice of $\beta$ depends only on $p)$, for all integer $\ell>k$, there exists a procedure to construct a multiplicative functional $\mathbf{y} \in \mathcal{T}_{\ell}(\mathrm{V})$ extending $\mathbf{x}$ (i.e., $\mathbf{x}^{i}=\mathbf{y}^{i}$ for $i=1, \ldots, k$ ) and satisfying (6.11) for $i=1, \ldots, \ell$. Moreover, the extension $\mathbf{y}$ of $\mathbf{x}$ given by this procedure is unique.

Sketch of the proof. The idea is to construct $\mathbf{y}^{(\ell)} \in \mathcal{T}_{\ell}(\mathrm{V})$ recursively, by setting $\mathbf{y}^{(k)}=\mathbf{x} \in \mathcal{T}_{k}(\mathrm{~V})$ and, once $\mathbf{y}^{(\ell)}$ has been defined, set $\mathbf{z}^{(\ell+1)}$ in $\mathcal{T}_{\ell+1}(\mathrm{~V})$ by $\mathbf{z}^{(\ell+1)}=$ $\mathbf{y}^{(\ell)}+\sum_{\left(i_{1}, \ldots, i_{\ell+1}\right)} 0 \cdot e_{i_{1}} \otimes e_{\ell+1}$. Thus, $\mathbf{y}^{(\ell+1)}$ is defined by

$$
\mathbf{y}_{s, t}^{(\ell+1)}=\lim _{\delta \rightarrow 0} \mathbf{z}_{t_{0}^{\delta}, t_{1}^{\delta}}^{(\ell+1)} \otimes \mathbf{z}_{t_{1}^{\delta}, t_{2}^{\delta}}^{(\ell+1)} \otimes \cdots \otimes \mathbf{z}_{t_{i^{\delta}-1}^{\delta}, t_{i}^{\delta} \delta}^{(\ell+1)},
$$

where $\Pi^{\delta}=\left\{t_{i}^{\delta} \mid s \leqslant t_{0}^{\delta} \leqslant \cdots \leqslant t_{i^{\delta}}^{\delta} \leqslant t\right\}$ is a partition of $[s, t]$ whose mesh goes to 0 as $\delta$ decreases to 0 . Thanks to the multiplicative property of $\mathbf{y}^{(\ell)}$, remark that $\mathbf{y}^{(\ell+1), i}=\mathbf{y}^{(\ell), i}$ for $i=1, \ldots, \ell$. In fact, this idea was already used in the proof of Proposition 4, and will be used later in the proof of Theorem 7. In $\mathcal{T}_{\ell}(\mathrm{V})$, the extension of $\mathbf{x}$ is then $\mathbf{y}^{(\ell)}$.

This Theorem means that there exists a function $\Psi_{p}$ transforming multiplicative functionals in $\mathcal{M}^{p}(V)$ to multiplicative functionals in $\mathcal{T}_{\infty}(\mathrm{V})$. There are many ways to extend a multiplicative functional. For example, if $\varphi$ is a smooth function with values in $\mathrm{V}^{\otimes 2}$, then $(1,0, \varphi(t)-\varphi(s))_{(s, t) \in \Delta^{+}}$belongs to $\mathcal{M}^{2}(\mathrm{~V})$ and extends the multiplicative functional $(1,0)$. But the function $\Psi_{1}$ applied to smooth paths yields the series of iterated integrals, i.e., $\Psi_{1}(x)_{s, t}=\Phi([s, t], x)$. Moreover, the next Theorem states that $\Psi_{p}$ is continuous on $\mathcal{M}^{p}(\mathrm{~V})$. So, given a multiplicative functional $\mathbf{x} \in \mathcal{M}^{p}(\mathrm{~V})$, we call $\Psi_{p}(\mathbf{x})$ its extension.

Theorem 6 ([32, Theorem 2.2.2]). Let $\mathbf{x}$ and $\mathbf{y}$ be two multiplicative functionals in $\mathcal{T}_{k}(\mathrm{~V})$ satisfying the hypotheses of Theorem 5 for the same $\omega$. Assume that there exists a constant $\beta$ large enough (depending only on $p$ ) and a constant $0<\varepsilon<1$ such that

$$
\left|\mathbf{x}_{s, t}^{i}-\mathbf{y}_{s, t}^{i}\right| \leqslant \varepsilon \frac{\omega(s, t)^{i / p}}{\beta \Gamma(i / p)} \text { for } i=1, \ldots, k .
$$

Then, their extensions $\widehat{\mathbf{x}}$ and $\widehat{\mathbf{y}}$ to $\mathcal{T}_{\infty}(\mathrm{V})$ given by Theorem $\mathbf{5}$ also satisfy (6.12) for all integer $i$. 
In view of Lemma 5, this means that the map giving the extension of a multiplicative functional in $\mathcal{T}_{\lfloor p\rfloor}(\mathrm{V})$ is continuous with respect to the norm $\|\cdot\|_{p}$.

Combined with Proposition 7 , this means that the extension of a geometric multiplicative functional in $\mathcal{G}^{p}(\mathrm{~V})$ to $\mathcal{T}_{\ell}(\mathrm{V})$ is also a geometric multiplicative functional in this space for all $\ell>\lfloor p\rfloor$.

Using Theorem 5, if $\mathbf{x}$ is a geometric multiplicative functional in $\mathcal{T}_{\lfloor p\rfloor}(\mathrm{V})$ of finite $p$-variation (i.e., $\|\mathbf{x}\|_{p}$ is finite: there is no difficulty to find a function $\omega$ such that $\mathbf{x}$ is controlled by $\omega)$, then one may solve (6.9) by density using (6.10) and the previous Theorem.

The idea behind Theorem 5 is that the more irregular is a trajectory ("rough"), the more "iterated integrals" have to be considered. But on the other side, once one knows enough iterated integrals, then the whole set of iterated integrals could be known. Hence, when one deals with a path $x$ of finite $p$-variation, then he needs to know a geometric multiplicative functional $\mathbf{x}$ lying above $x$ and belonging to the truncated tensor algebra $T_{\lfloor p\rfloor}(\mathrm{V})$.

\section{Almost multiplicative functionals}

When $p<2$, the geometric multiplicative functionals we consider are $\mathbf{x}_{s, t}=$ $\left(1, \mathbf{x}_{s, t}^{1}\right)$, with $\mathbf{x}_{s, t}^{1}=x_{t}-x_{s}$ for a continuous path of finite $p$-variation. In this case, we have defined integrals of the type $\int_{s}^{t} f\left(x_{r}\right) \mathrm{d} x_{r}$ for Hölder continuous functions $f$, which are, as we have seen, of finite $p$-variation. This integral is a multiplicative functional in $\mathcal{M}^{p}(\mathrm{~W})$, since from Chasles' relation,

$$
\int_{s}^{u} f\left(x_{r}\right) \mathrm{d} x_{r}+\int_{u}^{t} f\left(x_{r}\right) \mathrm{d} x_{r}=\int_{s}^{t} f\left(x_{r}\right) \mathrm{d} x_{r} .
$$

However, we have seen that $\int f\left(x_{r}\right) \mathrm{d} x_{r}$ may be constructed as limit of Riemann sums, and for that, we have used the approximation

$$
y_{s, t}=f\left(x_{s}\right)\left(x_{t}-x_{r}\right) \simeq \int_{s}^{t} f\left(x_{r}\right) \mathrm{d} x_{r} .
$$

Of course, $\left(y_{s, t}\right)_{(s, t) \in \Delta^{+}}$fails to satisfy (17.1), but the error $\varepsilon_{s, u, t}=y_{s, t}-y_{s, u}-y_{u, t}$ was easily controlled. And the estimate on $\varepsilon_{s, u, t}$ was the key of the proof of Proposition 1. So, $\left(y_{s, t}\right)_{(s, t) \in \Delta^{+}}$may be called an almost multiplicative functional.

Definition 3. A continuous function $\mathbf{x}: \Delta^{+} \rightarrow T_{k}(\mathrm{~V})$ for some integer $k$ is called an almost multiplicative functional if it is of finite $p$-variation controlled by $\omega$ (see Definition 2) and, for $i=1, \ldots, k$,

$$
\left|\left(\mathbf{x}_{s, t}-\mathbf{x}_{s, u} \otimes \mathbf{x}_{u, t}\right)^{i}\right| \leqslant K \omega(s, t)^{\theta} \text { for all } 0 \leqslant s \leqslant u \leqslant t \leqslant 1 .
$$

for some $\theta>1$ and some constant $K$. 
Thus, an almost multiplicative functional fails to satisfy (6.1b), but the error is in some sense close to a multiplicative functional.

Theorem 7 ([32, Theorem 3.3.1]). Let $\mathbf{x}$ be an almost multiplicative functional taking its values in $T_{k}(\mathrm{~V})$ with finite p-variation controlled by $\omega$. Then there exists a unique multiplicative functional $\mathbf{z}$ in $\mathcal{T}_{k}(\mathrm{~V})$ such that for all $(s, t) \in \Delta^{+}$, there exists a constant $C$ depending on $K, \theta$ (defined by (7.2)), the degree $k$ and the control $\omega(s, t)$ such that

$$
\left|\left(\mathbf{x}_{r, u}-\mathbf{z}_{r, u}\right)^{i}\right| \leqslant C \omega(r, u)^{\theta} \text { for } s \leqslant r \leqslant u \leqslant t \text { and } i=1, \ldots, k .
$$

Furthermore, there is at most one multiplicative functional $\mathbf{z}$ in $\mathcal{T}_{k}(\mathrm{~V})$ satisfying (7.3) regardless the choice of $C$.

Sketch of the proof. The idea was already used in the proofs of Proposition 4 and Theorem 5, Construct $\mathbf{z}$ by setting $\mathbf{z}^{0}=\mathbf{z}^{(0)}=1$ and recursively for $\ell=1, \ldots, k$, $\mathbf{y}_{s, t}^{(\ell)}=\mathbf{z}_{s, t}^{(\ell-1)}+\mathbf{x}_{s, t}^{\ell}$, where $\mathbf{z}^{(\ell-1)}=\left(\mathbf{z}^{0}, \mathbf{z}^{1}, \ldots, \mathbf{z}^{\ell-1}\right) \in \mathcal{T}_{\ell-1}(\mathrm{~V})$. Hence, define $\mathbf{z}^{(\ell)} \in \mathcal{T}_{\ell}(\mathrm{V})$ by

$$
\mathbf{z}_{s, t}^{(\ell)}=\lim _{\delta \rightarrow 0} \mathbf{y}_{t_{0}^{\delta}, t_{1}^{\delta}}^{(\ell)} \otimes \mathbf{y}_{t_{1}^{\delta}, t_{2}^{\delta}}^{(\ell)} \otimes \cdots \otimes \mathbf{y}_{t_{i^{\delta}-1}^{\delta}, t_{i^{\delta}}^{\delta}}^{(\ell)},
$$

where $\Pi^{\delta}=\left\{t_{i}^{\delta} \mid s \leqslant t_{0}^{\delta} \leqslant \cdots \leqslant t_{i^{\delta}}^{\delta} \leqslant t\right\}$ is a partition of $[s, t]$ whose mesh goes to 0 as $\delta$ decreases to 0 . The multiplicative functional $\mathbf{z}$ is then $\mathbf{z}^{(k)}$.

\section{Integration of a one form}

We now have all the elements to define an integral like $\int_{0}^{t} f\left(x_{s}\right) \mathrm{d} \mathbf{x}_{s}$ against a geometric multiplicative functional $\mathbf{x} \in \mathcal{T}_{\lfloor p\rfloor}(\mathrm{V})$ of finite $p$-variation for an arbitrary $p>1$. We want this integral to belong to $T_{\lfloor p\rfloor}(\mathrm{W})$ if $f$ is a one-form taking its values in a Banach space $\mathrm{W}$, and in fact that $(s, t) \in \Delta^{+} \mapsto \int_{s}^{t} f\left(x_{r}\right) \mathrm{d} \mathbf{x}_{r}$ belongs to $\mathcal{G}^{p}(\mathrm{~W})$.

\subsection{Lipschitz functions}

The notion of Lipschitz functions we use is that of E.M. Stein (see for example the book [44]).

Definition 4. Let $\mathrm{F}$ be a closed subset of the normed space $\mathrm{U}$, and $\alpha>0$. Let $\mathrm{W}$ be a separable Banach space, and $f$ a function with values in W. Assume that $k<\alpha \leqslant k+1$. Then $f$ belongs to $\operatorname{Lip}(\alpha, \mathrm{F}, \mathrm{W})$ if there exist some functions $f^{J}$ 
where $J$ is a multi-index of length length $(J) \leqslant k$ and some functions $R_{J}: \mathrm{F} \times \mathrm{F} \rightarrow$ W such that for all $x, y \in \mathrm{F}$,

$$
\begin{array}{r}
f^{J}(x)-f^{J}(y)=\sum_{\substack{L=\left(\ell_{1}, \ldots, \ell_{m}\right), \operatorname{length}(J, L) \leqslant k}} \frac{1}{\ell_{1} ! \cdots \ell_{m} !} f^{(J, L)}(y)\left(x^{\ell_{1}}-y^{\ell_{1}}\right) \cdots\left(x^{\ell_{m}}-y^{\ell_{m}}\right) \\
+R_{J}(x, y),
\end{array}
$$

where $(J, L)$ denotes the concatenation of the multi-indexes $J$ and $L$. By definition, $f^{\emptyset}=f$. The functions $f^{J}$ shall satisfy

$$
\left|f^{J}(x)\right| \leqslant M \text { and }\left|R_{J}(x, y)\right| \leqslant M|x-y|^{\alpha-\operatorname{length}(J)}
$$

for all $x, y \in \mathrm{F}$ and any $J$ of length length $(J) \leqslant k$. Denote by $\|f\|_{\text {Lip }}$ the smallest $M$ such that (8.1) is true. With this norm, $\operatorname{Lip}(\alpha, \mathrm{F}, \mathrm{W})$ is a Banach space.

This definition requires some comments. If $\mathrm{F}=\mathrm{V}=\mathbb{R}^{d}$, then the functions in $\operatorname{Lip}\left(\alpha, \mathbb{R}^{d}, \mathrm{~W}\right)$ are from $\mathbb{R}^{d}$ to $\mathrm{W}$ with bounded derivatives up to order $\lfloor\alpha\rfloor$. Moreover, $f^{\left(i_{1}, \ldots, i_{\ell}\right)}=\frac{\partial^{\ell} f}{\partial x_{i_{1}} \cdots \partial x_{i_{\ell}}}$, and $f^{\left.\left(i_{1}, \ldots, i_{\lfloor} \alpha\right\rfloor\right)}$ is $(\alpha-\lfloor\alpha\rfloor)$-Hölder continuous.

If $\mathrm{F}$ is a strict subset of $\mathbb{R}^{d}$, then a function $f \in \operatorname{Lip}(\alpha, \mathrm{F}, \mathrm{W})$ may be extended continuously to a function in $\operatorname{Lip}\left(\alpha, \mathbb{R}^{d}, \mathrm{~W}\right)$, but the family of the $f^{J}$ 's is not necessarily unique. In this case, by a function $f$ in $\operatorname{Lip}(\alpha, \mathrm{F}, \mathrm{W})$, we denote not only $f$, but the whole family $\left(f^{J}\right)_{J}$ multi-index, length $(J) \leqslant\lfloor\alpha\rfloor$.

\subsection{Integration}

To start with, let $f=\left(f_{1}, \ldots, f_{d}\right)$ be a smooth function defined on the Banach space $\mathrm{V}=\mathbb{R}^{d}$. The idea to define $\int_{0}^{t} f\left(x_{s}\right) \mathrm{d} \mathbf{x}_{s}$ is to construct an almost multiplicative function $\mathbf{y}$ such that $\mathbf{y}_{s, t}$ gives a first approximation of $\int_{s}^{t} f\left(x_{r}\right) \mathrm{d} x_{r}$, and then to transform $\mathbf{y}$ to a geometric multiplicative functional using Theorem 7 .

The value of $\mathbf{y}_{s, t}$ will be computed as previously using a Taylor expansion of $f$ when it is assumed that $x$ is smooth. So, in a first approach, assume that $x$ is piecewise smooth and that $\mathbf{x}$ is the geometric multiplicative functional given by its iterated integrals.

Set

$$
\begin{gathered}
y_{t}-y_{s}=\mathbf{y}_{s, t}^{1}=\sum_{j=1}^{k} \sum_{I \text { multi-index }, I=\left(i_{1}, \ldots, i_{j}\right)} \mathfrak{D}_{I}(f)\left(x_{s}\right) \int_{s}^{t} \mathrm{~d}_{I} x \\
\text { with } \mathfrak{D}_{I}(f)\left(x_{s}\right)=\frac{\partial^{j-1} f_{i_{1}}}{\partial x_{i_{j}} \cdots \partial x_{i_{2}}}\left(x_{s}\right) .
\end{gathered}
$$


Once this is done, one may define $\mathbf{y}^{j}$ for $j=2, \ldots, k$ using the iterated integrals of $y: \mathbf{y}_{s, t}^{j}=\sum_{I \text { multi-index, } I=\left(i_{1}, \ldots, i_{j}\right)} \int_{s}^{t} \mathrm{~d}_{I} y$. But this involves expressions such as

$$
S\left(J_{1}, \ldots, J_{\ell}\right)=\int_{s \leqslant s_{1} \leqslant \cdots \leqslant s_{\ell} \leqslant t} \mathrm{~d}\left(\int_{0}^{s_{1}} \mathrm{~d}_{J_{1}} x\right) \cdots \mathrm{d}\left(\int_{0}^{s_{\ell}} \mathrm{d}_{J_{\ell}} x\right),
$$

where $J_{1}, \ldots, J_{\ell}$ are themselves multi-indexes. But it is possible to express such a sum $S\left(J_{1}, \ldots, J_{\ell}\right)$ as the sum of

$$
S\left(J_{1}, \ldots, J_{\ell}\right)=\sum_{\substack{K \text { multi-index, } \\ \text { length } K=\text { length }\left(J_{1}\right)+\cdots+\text { length }\left(J_{\ell}\right)}} \varepsilon_{K} \int_{s}^{t} \mathrm{~d}_{K} x,
$$

where $\varepsilon_{K} \in\{0,1\}$ (see [32, Predefinition 3.2.1, p. 283]). Denote by $J_{1} \diamond \cdots \diamond J_{\ell}$ the set of multi-indexes $K$ that appear really in the previous sum, i.e., for which $\varepsilon_{K}=1$.

Definition 5. Let $\mathrm{x}$ be a geometric multiplicative functional of finite $p$-variation lying above a path $x$, and let $f$ be a differential form in $\operatorname{Lip}(\alpha, \mathrm{V}, \mathrm{W})$ for some $\alpha>p-1$, that is, $v \in \mathrm{V} \mapsto f(\cdot) v$ is linear and for all $v \in \mathrm{V}, f(\cdot) v$ belongs to $\operatorname{Lip}(\alpha, \mathrm{V}, \mathrm{W})$ for some $\alpha>p-1$. The integral $\int_{0}^{t} f\left(x_{s}\right) \mathrm{d} \mathbf{x}_{s}$ of $f$ along the path $\mathbf{x}$ is defined to be the geometric multiplicative functional of finite $p$-variation given by Theorem 7 corresponding to the almost multiplicative functional

$$
\mathbf{y}_{\substack{s, t \\ J_{1}, \ldots, J_{i} \\ \text { length }\left(J_{1}\right)+\cdots+\text { length }\left(J_{i}\right) \leqslant k}} \sum_{K \in J_{1} \diamond \cdots \diamond J_{i}} \mathfrak{D}_{J_{1}}(f)\left(x_{s}\right) \otimes \cdots \otimes \mathfrak{D}_{J_{i}}(f)\left(x_{s}\right) \mathbf{x}_{s, t}^{\operatorname{length}(K), K} .
$$

Here, $\mathfrak{D}_{J}(f)(x)$ is defined by to be an element in the dual of $\mathrm{V}^{\otimes \operatorname{length}(J)}$ (with values in W): If $e_{1}, \ldots, e_{d}$ is the canonical basis of $\mathrm{V}$ and $e_{1}^{\prime}, \ldots, e_{d}^{\prime}$ is its dual basis, then

$$
\mathfrak{D}_{\left(i_{1}, \ldots, i_{j}\right)}(f)(x)=f_{i_{1}}^{\left(i_{j}, \ldots, i_{2}\right)}(x) e_{i_{j}}^{\prime} \otimes \cdots \otimes e_{i_{1}}^{\prime} .
$$

Theorem 8 ([32, Theorem 3.2.1, p. 285]). Definition[5 is valid, i.e., the definition of $\mathbf{y}$ gives rise to an almost multiplicative functional, which is controlled by $K \omega$ if $\mathbf{x}$ is controlled by $\omega$, where the constant $K$ depends only on $\alpha, p,\|f\|_{\text {Lip }}$ and $\sup _{(s, t) \in \Delta^{+}} \omega(s, t)$. Moreover, $\mathbf{x} \mapsto \int f\left(x_{0}+\mathbf{x}_{0, s}^{1}\right) \mathrm{d} \mathbf{x}_{s}$ is continuous from $\mathcal{G}^{p}(\mathrm{~V})$ to $\mathcal{G}^{p}(\mathrm{~W})$.

\section{Solving differential equations}

We can now consider solving differential equations of the form

$$
y_{t}=a+\int_{0}^{t} f\left(y_{s}\right) \mathrm{d} \mathbf{x}_{s}
$$


where $\mathbf{x}$ belongs to $\mathcal{G}^{p}(\mathrm{~V})$, and $y_{t}$ belongs to a Banach space $\mathrm{W}$ for all $t \geqslant 0$. Thus, $f$ is a differential form, such that for all $v \in \mathrm{V}, f(\cdot) v$ belongs to $\operatorname{Lip}(\alpha, \mathrm{W}, \mathrm{W})$ for some $\alpha>p-1$.

Equation (9.1) will be solved using Picard's iteration principle: Construct a sequence $\left(y^{n}\right)_{n \in \mathbb{N}}$ by $y_{t}^{n+1}=a+\int_{0}^{t} f\left(y_{s}^{n}\right) \mathrm{d} \mathbf{x}_{s}$, and prove that $y^{n}$ converges to some element $y$. But such a principle requires that $y^{n+1}$ and $y^{n}$ belong to the same space. So, consider some multiplicative functional $\mathbf{z}=(\mathbf{x}, \mathbf{y})$ in $\mathcal{T}_{\lfloor p\rfloor}(\mathrm{V} \oplus \mathrm{W})$, and define

$$
\mathfrak{K}(\mathbf{z})=\int h\left(x_{0}+\mathbf{x}_{0, s}^{1}, a+\mathbf{y}_{0, s}^{1}\right) \mathrm{d} \mathbf{z}_{s},
$$

where $h(x, y)$ is the differential form $h(x, y)=\sum f_{i}(y) \mathrm{d} x^{i}$. Thus, Picard's iteration principle will be applied on elements of $\mathcal{T}_{\lfloor p\rfloor}(\mathrm{V} \oplus \mathrm{W})$.

Definition 6. The solution of (9.1) is an extension $\mathbf{z}$ in $\mathcal{G}^{p}(\mathrm{~V} \oplus \mathrm{W})$ of $\mathbf{x} \in \mathcal{G}^{p}(\mathrm{~V})$ such that $\mathbf{z}$ lies above $(x, y)$ with $\left(x_{0}, y_{0}\right)=\left(x_{0}, a\right)$ and $\mathbf{z}$ satisfies $\mathbf{z}=\mathfrak{K}(\mathbf{z})$.

Note that although the projection $\mathbf{y}$ on $\mathcal{G}^{p}(\mathrm{~W})$ of $\mathbf{z}$ can be seen as the solution of (9.1), $\mathbf{z}$ also keeps track of the "interactions" between $x$ and $y$ using the iterated integrals.

Theorem 9 ([32, Theorem 4.1.1, p. 298]). If $f$ is a linear form on $\mathrm{V}$ with values in $\operatorname{Lip}(\alpha, \mathrm{W}, \mathrm{W})$ for some $\alpha>p-1$, then there exists a solution to (9.1) when $\mathbf{x}$ belongs to $\mathcal{G}^{p}(\mathrm{~V})$. Moreover, if $f$ is a linear form on $\mathrm{V}$ with values in $\operatorname{Lip}(\alpha, \mathrm{W}, \mathrm{W})$ for $\alpha>p$, then this solution $\mathbf{z}$ is unique, and the map $\mathfrak{I}: \mathbf{x} \mapsto \mathbf{z}$, called the Itô map, is continuous from $\mathcal{G}^{p}(\mathrm{~V})$ to $\mathcal{G}^{p}(\mathrm{~V} \oplus \mathrm{W})$.

Remark 13. To prove the existence of a solution under the assumption that $f$ belongs to $\operatorname{Lip}(\alpha, \mathrm{W}, \mathrm{W})$ with $\alpha>p-1$, one only has to act as in Step 2 in the proof of Theorem 1: In Step 3 of the proof of Theorem 4.1.1 in [32], it is proved that the paths $y^{n}$ given by the Picard iteration principle are of finite $p$-variations controlled by the same $\omega$. Hence Lemma 8 can be used.

\section{A practical example: Brownian motion}

We show in this section how the theory of rough paths may be used to define a stochastic integral against Brownian motion. It is well known that almost surely, a trajectory of Brownian motion is $\alpha$-Hölder continuous for all $\alpha<1 / 2$. Thus, a trajectory of Brownian motion is then of finite $p$-variation for all $p>2$.

Given a Brownian trajectory $B(\omega)$, the main difficulty is to create a geometric multiplicative functional $\mathbf{B}(\omega)$ lying above $B$, where the Brownian motion is defined on a probability space $(\Omega, \mathcal{F}, \mathbb{P})$ and lives in $V=\mathbb{R}^{d}$. In view of Proposition 7 , 
one has only to construct a piecewise smooth approximation $B^{\delta}(\omega)$ converging to $B(\omega)$ as $\delta \rightarrow 0$, and to study the convergence of $\mathbf{B}^{\delta}$ with $\mathbf{B}^{1, \delta}(\omega)=B^{\delta}(\omega)$ and

$$
\mathbf{B}_{s, t}^{2, i, j, \delta}(\omega)=\int_{s}^{t} B_{r}^{i, \delta}(\omega) \mathrm{d} B_{r}^{j, \delta}(\omega)
$$

But we know that: (i) The limit of $\mathbf{B}^{2, i, j, \delta}$ depends on the choice of the approximation. (ii) When it converges, $\mathbf{B}^{2, i, j, \delta}$ does not converge almost surely but only in probability or in $\mathrm{L}^{2}(\mathbb{P})$ (however, it is proved that for dyadic partitions, the convergence may be almost sure. See [24] for example).

Point (ii) is contained in the classical result from E. Wong and M. Zakai in [51] for some piecewise linear approximation of the Brownian motion, while point (i) is related to the extensions of such a result (see [19, Sect. VI,-7, p. 392] or [22, Chap. 5.7, p. 274] for example). In fact, the problems with (i) are similar to the results given in Sect. 6.2. There are different geometric multiplicative functionals lying above the same path $B$.

\subsection{The "natural" choice}

The natural choice for $B^{\delta}$ is given by

$$
B_{t}^{\delta}(\omega)=B_{t_{i}}(\omega)+\left(t_{i+1}-t_{i}\right)^{-1}\left(t-t_{i}\right)\left(B_{t_{i+1}}(\omega)-B_{t_{i}}(\omega)\right)
$$

for $t \in\left[t_{i}, t_{i+1}\right]$, where $\Pi^{\delta}=\left\{t_{i} \mid 0 \leqslant t_{1} \leqslant \cdots \leqslant t_{k} \leqslant 1\right\}$ is a deterministic partition $[0,1]$ whose mesh goes to 0 with $\delta$. It is clear that $B^{\delta}(\omega)$ converges uniformly to $B(\omega)$. We have seen at the end of Sect. 2.3 that $\operatorname{Var}_{q,[0,1]}\left(B^{\delta}(\omega)\right) \leqslant$ $3 \operatorname{Var}_{q,[0,1]}(B(\omega))$ for all $q>2$. According to Lemma $8, B^{\delta}(\omega)$ converges in the topology generated by $\operatorname{Var}_{p,[0,1]}(\cdot)+\|\cdot\|_{\infty}$ to $B(\omega)$ for all $p>2$.

For such an approximation, we know that

$$
\mathbf{B}_{s, t}^{2, i, j, \delta} \stackrel{\text { uniformly in }(s, t) \in \Delta^{+}}{\longrightarrow} \mathbf{B}_{s, t}^{2, i, j} \stackrel{\text { def }}{=} \int_{s}^{t}\left(B_{r}^{i}-B_{s}^{i}\right) \circ \mathrm{d} B_{r}^{j}
$$

in probability. Here, the stochastic integral is a Stratonovich integral. We prove below in Sect. 11.2 that $\mathbf{B}^{\delta}$ converges to $\mathbf{B}$ in the topology generated by $\|\cdot\|_{p}$ if the partitions $\Pi^{\delta}$ are dyadic. Thus, for this choice of $\mathbf{B}^{2}$, the geometric multiplicative functional $\mathbf{B}$ belongs to $\mathcal{G}^{p}(\mathrm{~V})$ for all $p>2$.

Now, let $f$ be a linear function on $\mathrm{V}$ taking its values in $\operatorname{Lip}(\alpha, \mathrm{V}, \mathrm{W})$ for some $\alpha>1$, with $\mathrm{W}=\mathbb{R}^{m}$. A direct consequence of Proposition 3 or Theorem 8 is that $\mathbf{X}_{s, t}=\int_{s}^{t} f\left(B_{r}\right) \mathrm{d} \mathbf{B}_{r}$ is well defined and belongs to $\mathcal{G}^{p}(\mathrm{~W})$ for all $p \in(2,1+\alpha)$.

Remark 14. A practical feature of the theory of rough paths is that $\mathbf{X}$ is defined on a subset $\Omega_{0} \subset \Omega$ of full measure whatever the function $f$ is. 
Let $X$ be a path such that $\mathbf{X}$ lies above $X$, with $X_{0}=x_{0}$. Then, a direct consequence of Theorem 8 is that

$$
X_{t}^{\delta}=x_{0}+\int_{0}^{t} f\left(B_{s}^{\delta}\right) \mathrm{d} B_{s}^{\delta} \underset{\delta \rightarrow 0}{\stackrel{\text { probability }}{\longrightarrow}} X_{t},
$$

the convergence holding with respect to both the uniform norm and the norm of $p$-variation. But from a theorem of Wong-Zakai type, it is also known that $X^{\delta}$ converges in probability to $x_{0}+\int_{0}^{\cdot} f\left(B_{s}\right) \circ \mathrm{d} B_{s}$. Thus, this integral is almost surely equal to $X$. There is in fact a deep relation between the Stratonovich integral and the one given by the theory of rough paths.

\subsection{Stratonovich integrals and rough paths theory}

We develop in this section the link between stochastic integrals given by the theory of rough paths and Stratonovich integrals for Brownian motion. It also explains the influence of the term $\mathbf{B}^{2}$, where $\mathbf{B}$ is a geometric multiplicative functional lying above the Brownian motion, but different from the one given by the "natural" construction of Sect. 10.1. Note that such geometric multiplicative functionals may arise naturally. For example (see e.g., [26, 28] in the homogenization theory), there exist some families $\left(X^{\varepsilon}\right)_{\varepsilon>0}$ of semi-martingales converging, thanks to a central limit theorem, to a Brownian motion $B$, but such that $A_{s, t}\left(X^{\varepsilon}\right)$ converges to $A_{s, t}(B)+c(t-s)$ for some matrix $c$. of

By definition, the Stratonovich integral $\int_{0}^{t} f_{j}\left(B_{s}\right) \circ \mathrm{d} B_{s}^{j}$ is the limit in probability

$$
\begin{aligned}
I^{\delta} & \stackrel{\text { def }}{=} \sum_{i=1}^{k-1} \frac{\left(f_{j}\left(B_{t_{i+1}}\right)+f_{j}\left(B_{t_{i}}\right)\right)}{2}\left(B_{t_{i+1}}^{j}-B_{t_{i}}^{j}\right) \\
& =\sum_{i=1}^{k-1} f_{j}\left(B_{t_{i}}\right)\left(B_{t_{i+1}}^{j}-B_{t_{i}}^{j}\right)+\sum_{i=1}^{k-1} \frac{\left(f_{j}\left(B_{t_{i+1}}\right)-f_{j}\left(B_{t_{i}}\right)\right)}{2}\left(B_{t_{i+1}}^{j}-B_{t_{i}}^{j}\right)
\end{aligned}
$$

where $\Pi^{\delta}=\left\{t_{i} \mid 0 \leqslant t_{1} \leqslant \cdots \leqslant t_{k} \leqslant t\right\}$ is a deterministic partition of $[0, t]$. The functions $f$ belongs to $\operatorname{Lip}(\alpha, \mathrm{W}, \mathrm{W})$ with $\alpha>1$. Let $p \in(2,1+\alpha)$ be fixed. By definition, $f_{j}(x)-f_{j}(y)=\sum_{\ell=1}^{d} \frac{\partial f_{j}}{\partial x_{\ell}}\left(y^{\ell}-x^{\ell}\right)+R_{j}(x, y)$ and $R_{j}(x, y) \leqslant|y-x|^{\alpha}$. Thus,

$$
\begin{aligned}
I^{\delta}= & \sum_{i=1}^{k-1} f_{j}\left(B_{t_{i}}\right)\left(B_{t_{i+1}}^{j}-B_{t_{i}}^{j}\right) \\
& +\frac{1}{2} \sum_{i=1}^{k-1} \sum_{\ell=1}^{d} \frac{\partial f_{j}}{\partial x_{\ell}}\left(B_{t_{i}}\right)\left(B_{t_{i+1}}^{\ell}-B_{t_{i}}^{\ell}\right)\left(B_{t_{i+1}}^{j}-B_{t_{i}}^{j}\right)+\varepsilon^{\delta}
\end{aligned}
$$


with

$$
\begin{aligned}
\varepsilon^{\delta} & =\sum_{i=1}^{k-1} R_{j}\left(B_{t_{i}}, B_{t_{i+1}}\right)\left(B_{t_{i+1}}^{j}-B_{t_{i}}^{j}\right) \\
& \leqslant C \sum_{i=1}^{k-1}\left|t_{i+1}-t_{i}\right|^{(\alpha+1) / p} \leqslant C t \sup _{i=1, \ldots, k-1}\left|t_{i+1}-t_{i}\right|^{(\alpha+1-p) / p} \underset{\delta \rightarrow 0}{\longrightarrow} 0 .
\end{aligned}
$$

This constant $C$ is such that $\left|B_{t}-B_{s}\right| \leqslant C^{1 /(1+\alpha)}|t-s|^{1 / p}$ for the considered trajectory of the Brownian motion.

Now, let $\mathbf{B}$ be a geometric multiplicative functional lying above $B$. There is no necessity to choose the previous one, and we have seen in Sect. 6.2 how to construct as many areas as we want. Then

$$
\frac{1}{2}\left(B_{t_{i+1}}^{\ell}-B_{t_{i}}^{\ell}\right)\left(B_{t_{i+1}}^{j}-B_{t_{i}}^{j}\right)=\mathbf{B}_{t_{i}, t_{i+1}}^{2, \ell, j}-\mathfrak{A}_{t_{i}, t_{i+1}}^{\ell, j}(\mathbf{B}),
$$

where $\mathfrak{A}(\mathbf{B})$ is the antisymmetric part of $\mathbf{B}^{2}$. Moreover, we have seen that the sum $\sum_{j=1}^{d} \sum_{i=1}^{k-1} f_{j}\left(B_{t_{i}}\right)\left(B_{t_{i+1}}^{j}-B_{t_{i}}^{j}\right)+\frac{1}{2} \frac{\partial f_{j}}{\partial x_{\ell}}\left(B_{t_{i}}\right) \mathbf{B}_{t_{i}, t_{i+1}}^{2, \ell, j}$ converges almost surely to $X_{t}-X_{0}$, where $X$ is the path above which $\int f\left(B_{s}\right) \mathrm{d} \mathbf{B}_{s} \in \mathcal{G}^{p}(\mathrm{~W})$ lies. So, we deduce that

$$
\begin{gathered}
X_{0}+\sum_{j=1}^{d} \int_{0}^{t} f_{j}\left(B_{s}\right) \circ \mathrm{d} B_{s}^{j}=X_{t}-\mathfrak{Q}_{t}(\mathbf{B}) \\
\text { with } \mathfrak{Q}_{t}(\mathbf{B})=\lim _{\delta \rightarrow 0} \sum_{j, \ell=1}^{d} \sum_{i=1}^{k} \frac{\partial f_{j}}{\partial x_{\ell}}\left(B_{t_{i}}^{\delta}\right) \mathfrak{A}_{t_{i}, t_{i+1}}^{\ell, j}(\mathbf{B}) .
\end{gathered}
$$

The limit defining $\mathfrak{Q}_{t}(\mathbf{B})$ is a limit in probability.

Remark 15. Using the antisymmetry of $\mathfrak{A}_{s, t}^{\ell, j}(\mathbf{B})$, one has

$$
\mathfrak{Q}_{t}(\mathbf{B})=\lim _{\delta \rightarrow 0} \frac{1}{2} \sum_{j, \ell=1}^{d} \sum_{i=1}^{k-1}\left(\frac{\partial f_{j}}{\partial x_{\ell}}-\frac{\partial f_{\ell}}{\partial x_{j}}\right)\left(B_{t_{i}}^{\delta}\right) \mathfrak{A}_{t_{i}, t_{i+1}}^{\ell, j}(\mathbf{B}) .
$$

Thus, if $\frac{\partial f_{j}}{\partial x_{\ell}}-\frac{\partial f_{\ell}}{\partial x_{j}}=0, \mathfrak{Q}_{t}(\mathbf{B})=0$, then $X$ depends only on $B$ and not on the choice of $\mathbf{B}^{2}$. In particular, this is true if $f_{i}=\frac{\partial F}{\partial x_{i}}$ for some function $F$. In such a case, this could be shown directly, if $\left(\mathbf{B}^{\delta}\right)_{\delta>0}$ is a family of geometric multiplicative function lying above an approximation $B^{\delta}$ of $B$ and converging to $\mathcal{G}^{p}(\mathrm{~V})$ to $\mathbf{B}$, then the change of variables' formula reads: $F\left(B_{t}^{\delta}\right)-F\left(B_{0}^{\delta}\right)=\int_{0}^{t} f_{i}\left(B_{s}^{\delta}\right) \mathrm{d} B_{s}^{\delta}$. Thus, $F\left(B_{t}^{\delta}\right)-F\left(B_{0}^{\delta}\right)$ converges to $F\left(B_{t}\right)-F\left(B_{0}\right)$, while $\int_{0}^{t} f_{i}\left(B_{s}^{\delta}\right) \mathrm{d} B_{s}^{\delta}$ converges to $X_{t}$.

Now, if $\mathbf{B}^{\text {nat }}$ is the "natural" rough path lying above $B$ (see Sect. 10.1), then $\mathfrak{A}_{s, t}^{\ell, j}\left(\mathbf{B}^{\text {nat }}\right)$ is the Lévy area $A_{s, t}^{\ell, j}(B)$ of the 2 -dimensional Brownian motion $\left(B_{r}^{\ell}, B_{r}^{j}\right)_{r \in[s, t]}$, i.e., the area enclosed between the curve of $\left(B^{\ell}, B^{j}\right)$ and its chord:

$$
A_{s, t}^{\ell, j}(B)=\frac{1}{2}\left(\int_{s}^{t}\left(B_{r}^{\ell}-B_{s}^{\ell}\right) \circ \mathrm{d} B_{r}^{j}-\int_{s}^{t}\left(B_{r}^{j}-B_{s}^{j}\right) \circ \mathrm{d} B_{r}^{\ell}\right) .
$$


The result given at the end of Sect. 10.1 implies that $\mathfrak{Q}_{t}\left(\mathbf{B}^{\text {nat }}\right)=0$ almost surely.

Moreover, one knows from Sect.6.2 that there exists a function $\varphi=\left(\varphi_{i, j}\right)_{i, j=1, \ldots, d}$ from $[0,1]$ to the space of antisymmetric matrices (i.e., $\varphi_{i, j}(t)=-\varphi_{j, i}(t)$ for all $t \in[0,1])$ and of finite $p / 2$-variation such that $\mathfrak{A}_{s, t}(\mathbf{B})=A_{s, t}(B)+\varphi(t)-\varphi(s)$. We deduce that

$$
\mathfrak{Q}_{t}(\mathbf{B})=\sum_{j, \ell=1}^{d} \int_{0}^{t} \frac{\partial f_{j}}{\partial x_{\ell}}\left(B_{s}\right) \mathrm{d} \varphi_{\ell, j}(s) .
$$

To summarize, if $B^{\delta}$ is a piecewise smooth approximation of $B$ such that $\left(1, B^{\delta}, \int \mathrm{d} B^{\delta} \otimes \mathrm{d} B^{\delta}\right)$ converges to the geometric multiplicative functional $\mathbf{B}$ in $\mathcal{G}^{p}(\mathrm{~V})$, and $\mathfrak{A}_{s, t}(\mathbf{B})=A_{s, t}(B)+\varphi(t)-\varphi(s)$, then we obtain directly that

$$
\begin{aligned}
& \int_{0}^{t} f\left(B_{s}^{\delta}\right) \mathrm{d} B_{s}^{\delta} \stackrel{\text { probability }}{\longrightarrow} \int_{0}^{t} f\left(B_{s}\right) \circ \mathrm{d} B_{s}+\sum_{j, \ell=1}^{d} \int_{0}^{t} \frac{\partial f_{j}}{\partial x_{\ell}}\left(B_{s}\right) \mathrm{d} \varphi_{\ell, j}(s) \\
&=\int_{0}^{t} f\left(B_{s}\right) \circ \mathrm{d} B_{s}+\frac{1}{2} \sum_{j, \ell=1}^{d} \int_{0}^{t}\left(\frac{\partial f_{j}}{\partial x_{\ell}}-\frac{\partial f_{\ell}}{\partial x_{j}}\right)\left(B_{s}\right) \mathrm{d} \varphi_{\ell, j}(s) .
\end{aligned}
$$

When one considers the solution $Y^{\delta}$ in $\mathrm{W}=\mathbb{R}^{m}$ of the ordinary differential equations

$$
Y_{t}^{\delta}=y_{0}+\int_{0}^{t} f\left(Y_{s}^{\delta}\right) \mathrm{d} B_{s}^{\delta},
$$

then $Y^{\delta}$ converges in probability to the solution $Y$ of

$$
Y_{t}=y_{0}+\int_{0}^{t} f\left(Y_{s}\right) \circ \mathrm{d} B_{s}+\frac{1}{2} \sum_{\substack{\ell, k=1, \ldots, m \\ j=1, \ldots, d}} \int_{0}^{t}\left(\frac{\partial f_{j}}{\partial x_{\ell}} f_{\ell}^{k}-\frac{\partial f_{j}}{\partial x_{k}} f_{k}^{\ell}\right)\left(Y_{s}\right) \mathrm{d} \varphi_{k, \ell}(s) .
$$

Here, the drift term is different from the one in (10.2), since it comes from the cross iterated integrals of the type $\int \mathrm{d} Y \otimes \mathrm{d} B$, which may be computed first for smooth paths, and then by passing to the limit.

Thus, the theory of rough paths provides us with some new light on the results presented in Sect. VI-7 in [19, p. 392] (see also Historical Note 5 below). In this book, the results concern the case where $\varphi_{\ell, j}(t)=c_{\ell, j}$ where $c=\left(c_{\ell, j}\right)_{\ell, j=1, \ldots, d}$ is an antisymmetric matrix, whose terms are given by

$$
c_{\ell, j}=\lim _{\delta \rightarrow 0} \frac{1}{2 \delta} \mathbb{E}\left[\int_{0}^{\delta} B_{s}^{\ell, \delta} \mathrm{d} B_{s}^{j, \delta}-\int_{0}^{\delta} B_{s}^{j, \delta} \mathrm{d} B_{s}^{\ell, \delta}\right]=\lim _{\delta \rightarrow 0} \frac{1}{2 \delta} \mathbb{E}\left[A_{0, \delta}^{\ell, j}\left(B^{\delta}\right)\right],
$$

where $B^{\delta}(\omega)$ is given by an interpolation of $B(\omega)$ sampled at points $0, \delta, 2 \delta, \ldots$ The matrix $c$ depends on the way the trajectory is interpolated. 


\section{A. Lejay / An Introduction to Rough Paths}

Remark 16. In the construction of the Stratonovich integrals, it is important that the partitions $\Pi^{\delta}$ are deterministic. If not, a drift term may appear, which may be computed using the expression of $\mathfrak{Q}_{t}(B)$. This result has also been widely used to construct approximations of solutions of SDEs using partitions whose meshed goes to 0 at a speed that depends on the considered trajectory of the underlying Brownian motion (see for example [16]).

\subsection{Itô stochastic integrals}

When one construct integrals, only the fact that $\mathbf{x}$ is multiplicative is used. The fact that $\mathbf{x}$ is geometric is not really used, except that is allows us to construct first the objects for smooth paths, and then to deduce what the result should be for general geometric multiplicative functionals. It is the way the integral was defined in Sect. 8. However, when $p<3$, as we have seen in Sect. 3, one may directly set, given a multiplicative functional $\mathbf{x}$,

$$
\mathbf{y}_{s, t}^{1}=f\left(x_{s}\right) \mathbf{x}_{s, t}^{1}+\nabla f\left(x_{s}\right) \mathbf{x}_{s, t}^{2} \text { and } \mathbf{y}_{s, t}^{2}=f\left(x_{s}\right) \otimes f\left(x_{s}\right) \mathbf{x}_{s, t}^{2},
$$

and prove that $\mathbf{y}=\left(\mathbf{y}^{1}, \mathbf{y}^{2}\right)$ may be transformed into a multiplicative functional denoted by $\int f\left(x_{s}\right) \mathrm{d} \mathbf{x}_{s}$. Moreover, the map $\mathbf{x} \mapsto \int f\left(x_{0}+\mathbf{x}_{0, s}\right) \mathrm{d} \mathbf{x}_{s}$ is continuous in $\mathcal{M}^{p}(\mathrm{~V})$.

For a $N$-dimensional Brownian motion $B$, we know that

$$
\int_{s}^{t}\left(B_{r}^{i}-B_{s}^{i}\right) \circ \mathrm{d} B_{s}^{j}=\int_{s}^{t}\left(B_{r}^{i}-B_{s}^{i}\right) \mathrm{d} B_{s}^{j}+\delta_{i, j}(t-s)
$$

where $\delta_{i, j}$ is the Kronecker symbol. Thus, one may define a multiplicative functional $\mathbf{B}^{\text {itô }} \in \mathcal{M}^{p}(\mathrm{~V})$ by $\left(\mathbf{B}_{s, t}^{\text {itô }}\right)^{1, i}=B_{t}^{i}-B_{s}^{i},\left(\mathbf{B}_{s, t}^{\text {itô }}\right)^{2, i, j}=\left(\mathbf{B}_{s, t}^{\text {nat }}\right)^{2, i, j}-\delta_{i, j}(t-s)$ and define the (pathwise) Itô stochastic integral to be $\int f\left(B_{s}\right) \mathrm{d} \mathbf{B}_{s}^{\text {itô }}$.

Remark 17. Of course, $\int f\left(B_{s}\right) \mathrm{d} \mathbf{B}_{s}$ and $\int f\left(B_{s}\right) \mathrm{d} \mathbf{B}_{s}^{\text {itô }}$ are defined pathwise, but pathwise means "pathwise with respect to $\mathbf{B}$ " and not pathwise with respect to $B$ ". And the definition of $\mathbf{B}$ from $B$ is not pathwise, and requires that some stochastic integration, here of Itô or Stratonovich type, is already defined.

\section{How to compute $p$-variation?}

It is generally difficult to compute the $p$-variation of a function. We give in this section a trick which has been introduced in [17] and allows us to compute the $p$-variation of a multiplicative functional $\mathbf{x}$ provided one has a nice estimate on $\left|\mathbf{x}_{i 2^{-n},(i+1) 2^{-n}}^{j}\right|^{p / j}$ for $j=1, \ldots,\lfloor p\rfloor$, for all integer $n$ and $i=0, \ldots, 2^{n}-1$.

We give an example in the case of the Brownian motion, that allows us to complete the results of Sect. 10.1. In fact, this approach was successful in many cases: See [38, 3, 24, 1, 27] for various applications. 


\subsection{Using dyadics}

For any integer $n$ and any $k=0, \ldots, 2^{n}$, set $t_{k}^{n}=i / 2^{n}$, that is $\left(t_{k}^{n}\right)_{k=0, \ldots, 2^{n}}$ is the dyadic partition of $[0,1]$ at level $n$. Let $(s, t)$ belongs to $\Delta^{+}$, and construct recursively a sequence $\left(s_{m}\right)_{m \in \mathbb{Z}, m \neq 0}$ of elements in $\left(t_{k}^{n}\right)_{n \in \mathbb{N}, k=0, \ldots, 2^{n}}$ by the following way: Let $n_{0}$ be the smallest integer such that $\left[t_{k}^{n_{0}}, t_{k+1}^{n_{0}}\right] \subset[s, t]$ for some integer $k$. set $s_{-1}=t_{k}^{n_{0}}$ and $s_{1}=t_{k+1}^{n_{0}}$. Hence, construct $s_{m}$ for $m \geqslant 1$ by setting, if $s_{m}<t$,

$$
n_{m}=\inf \left\{n \geqslant n_{m-1} \mid \exists k \in \mathbb{N}, t_{k}^{n_{m}}=t_{k}^{n_{m-1}}, t_{k+1}^{m_{n}} \leqslant t\right\} .
$$

Denote by $s_{m}$ the value $t_{k+1}^{n_{m}}$, where $k$ is the unique integer for which $t_{k}^{n_{m}}=t_{k}^{n_{m-1}}$ and $t_{k+2}^{n_{m}}>t$. If $s_{m}=t$ then $s_{n}=t$ for all $n \geqslant m$.

Construct $s_{m}$ for $m<-1$ using a similar procedure, where $s_{m}$ decreases to $s$ instead of increasing to $t$.

This construction ensures that the sequences $\left(n_{m}\right)_{m \in \mathbb{N}}$ and $\left(n_{m}\right)_{m \in \mathbb{Z}^{*}, m<0}$ are increasing, and

$$
[s, t]=\cdots \cup\left[s_{-m-1}, s_{-m}\right] \cup \cdots \cup\left[s_{-1}, s_{1}\right] \cup \cdots \cup\left[s_{m-1}, s_{m}\right] \cup \cdots .
$$

Then, for all $\mathbf{x}$ in $\mathcal{M}^{p}(\mathrm{~V})$,

$$
\mathbf{x}_{s, t}=\lim _{m \rightarrow \infty} \mathbf{x}_{s_{-m-1}, s_{-m}} \otimes \cdots \otimes \mathbf{x}_{s_{-1}, s_{1}} \otimes \cdots \otimes \mathbf{x}_{s_{m}, s_{m+1}} .
$$

Thus, if $k=\lfloor p\rfloor$ and $j=1, \ldots, k$,

$$
\mathbf{x}_{s, t}^{j}=\sum_{i=1}^{j} \sum_{\substack{r_{1}, \ldots, r_{i}=1, \ldots, j \\ r_{1}+\cdots+r_{i}=j}} \sum_{\substack{m_{1}<\cdots<m_{i} \\ m_{1}, \ldots, m_{i} \in \mathbb{Z}^{*}}} \mathbf{x}_{s_{m_{1}}, s_{m_{1}+1}}^{r_{1}} \otimes \cdots \otimes \mathbf{x}_{s_{m_{i}}, s_{m_{i}+1}}^{r_{i}} .
$$

In the previous expression, we use the convention that $m_{i}+1=1$ if $m_{i}=-1$. So, for $j=1, \ldots, k$,

$$
\left|\mathbf{x}_{s, t}^{j}\right| \leqslant \sum_{i=1}^{j} \sum_{\substack{r_{1}, \ldots, r_{i}=1, \ldots, j \\ r_{1}+\cdots+r_{i}=j}}\left(\sum_{m \in \mathbb{Z}^{*}}\left|\mathbf{x}_{s_{m}, s_{m+1}}^{r_{1}}\right|\right) \cdots\left(\sum_{m \in \mathbb{Z}^{*}}\left|\mathbf{x}_{s_{m}, s_{m+1}}^{r_{i}}\right|\right) .
$$

Using the Hölder inequality, for $r=1, \ldots, k$ and any $\beta>(p-1) / p$,

$$
\begin{aligned}
\sum_{m \in \mathbb{Z}^{*}}\left|\mathbf{x}_{s_{m}, s_{m+1}}^{r}\right| & \leqslant C(r)\left(\sum_{m \in \mathbb{Z}^{*}} n_{m}^{\beta p / r}\left|\mathbf{x}_{s_{m}, s_{m+1}}^{r}\right|^{p / r}\right)^{r / p} \\
& \leqslant C\left(\sum_{r=1}^{j} \sum_{m \in \mathbb{Z}^{*}} n_{m}^{\beta p / r}\left|\mathbf{x}_{s_{m}, s_{m+1}}^{r}\right|^{p / r}\right)^{r / p},
\end{aligned}
$$


where $C(r)=\left(\sum_{m \in \mathbb{Z}^{*}} n_{m}^{-\beta p /(p-r)}\right)^{(p-r) / p}$ and $C=\sup _{r=1, \ldots, k} C(r)$. Our choice of $\beta$ ensures that $C$ is finite. Then, there exists a constant $K$ depending only on $k$ and $C$ such that

$$
\left|\mathbf{x}_{s, t}^{j}\right| \leqslant K\left(\sum_{r=1}^{j} \sum_{m \in \mathbb{Z}^{*}} n_{m}^{\beta p / r}\left|\mathbf{x}_{s_{m}, s_{m+1}}^{r}\right|^{p / r}\right)^{j / p}
$$

and then that

$$
\left|\mathbf{x}_{s, t}^{j}\right| \leqslant K\left(\sum_{r=1}^{j} \sum_{n \geqslant n(s, t)} n^{\beta p / r} \sum_{\substack{i=0, \ldots, 2^{n}-1 \\ t_{i}^{n} \in[s, t], t_{i+1}^{n} \in[s, t]}}\left|\mathbf{x}_{t_{i}^{n}, t_{i+1}^{n}}^{r}\right|^{p / r}\right)^{j / p},
$$

where $n(s, t)$ is the smallest integer $n$ such that there exists some integer $k$ for which $\left[t_{k}^{n}, t_{k+1}^{n}\right] \subset[s, t]$.

Inequality (11.1) is useful, since it allows to estimate both

$$
\sup _{(s, t) \in \Delta^{+}, t-s<\eta}\left|\mathbf{x}_{s, t}^{j}\right|, \text { and } \operatorname{Var}_{p / j,[0,1]}\left(\mathbf{x}^{j}\right)
$$

which satisfies

$$
\operatorname{Var}_{p / j,[0,1]}\left(\mathbf{x}^{j}\right) \leqslant K\left(\sum_{r=1}^{j} \sum_{n \geqslant 0} n^{\beta p / r} \sum_{i=0}^{2^{n}-1}\left|\mathbf{x}_{t_{i}^{n}, t_{i+1}^{n}}^{r}\right|^{p / r}\right)^{j / p}
$$

provided one knows $\mathbf{x}_{t_{i}^{n}, t_{i+1}^{n}}$ for all dyadic point $t_{i}^{n}=i / 2^{n}$.

\subsection{Application to Brownian motion}

We have constructed in Sect. 10.1 a piecewise linear approximation $B^{\delta}(\omega)$ of a Brownian motion trajectory $B(\omega)$. Let $\mathbf{B}^{\delta}$ (resp. B) be the geometric multiplicative functional in $\mathcal{G}^{p}(\mathrm{~V})$ lying above $B^{\delta}$ (resp. $B$ ) and constructed as in Sect. 10.1,

Proposition 8. The sequence $\left(\mathbf{B}^{\delta}\right)_{\delta>0}$ converges in probability to $\mathbf{B}$ in $\mathcal{M}^{p}\left(\mathbb{R}^{d}\right)$ for all $2 \leqslant p<3$. Moreover, if $\mathbf{B}^{\delta}$ is a piecewise linear approximation of $\mathbf{B}$ along dyadic partitions (consider only the $\delta$ 's of type $1 / 2^{n}$ ), then $\mathbf{B}^{\delta}$ converges almost surely to $\mathbf{B}$ in $\mathcal{M}^{p}\left(\mathbb{R}^{d}\right)$.

Proof. To simplify, we do not give a complete proof here. We prove only that $\mathbf{B}^{\delta}$ converges in probability to $\mathbf{B}$ where $\delta=2^{-n}$ for some integer $n$, and the partition $\Pi^{\delta}$ we use is $\left(t_{k}^{n}\right)_{k=0, \ldots, 2^{n}}$ with $t_{k}^{n}=k 2^{-n}$. 
There is no real difficulties in extending the proof when $\Pi^{\delta}$ is not dyadic partitions (see [27] for example), although it requires a bit more computations. Furthermore, still using the ideas to compute the $p$-variation of $\mathbf{B}^{\delta}$ by estimating $\mathbf{B}_{s, t}^{\delta}$, where $s, t$ are dyadic points $(s, t)=\left(k / 2^{m},(k+1) / 2^{m}\right)$ for all $m \geqslant 1$ and any $k \in\left\{0, \ldots, 2^{n}\right\}$, it could be shown that if $\Pi^{\delta}$ is the dyadic partition, then $\mathbf{B}^{\delta}$ converges almost surely to $\mathbf{B}$ (see [24]).

Let $q$ be a real number in $(2, p)$. If $m \leqslant n$, then according to the Doob inequality, there exists a constant $C$ depending only on $q$ such that for $a=1, \ldots, d$,

$$
\mathbb{E}\left[\left|B_{t_{k+1}^{m}}^{a, \delta}-B_{t_{k}^{m}}^{a, \delta}\right|^{q}\right] \leqslant \mathbb{E}\left[\left|B_{t_{k+1}^{m}}^{a}-B_{t_{k}^{m}}^{a}\right|^{q}\right] \leqslant C\left(t_{k+1}^{m}-t_{k}^{m}\right)^{q / 2} \leqslant C 2^{-m q / 2} .
$$

If $m>n$, then again by the Doob inequality, there exists a constant $C$ depending only on $q$ such that for $a=1, \ldots, d$,

$$
\begin{aligned}
\mathbb{E}\left[\left|B_{t_{k+1}^{m}}^{a, \delta}-B_{t_{k}^{m}}^{a, \delta}\right|^{q}\right] & \leqslant\left|\frac{t_{k+1}^{m}-t_{k}^{m}}{t_{i+1}^{n}-t_{i}^{n}}\right|^{q} \mathbb{E}\left[\left|B_{t_{i}^{n}}^{a, \delta}-B_{t_{i}^{n}}^{a, \delta}\right|^{q}\right] \\
& \leqslant C 2^{-m q} 2^{n q / 2} \leqslant C 2^{-m q / 2}
\end{aligned}
$$

if $i$ is such that $\left[t_{k}^{m}, t_{k+1}^{m}\right] \subset\left[t_{i}^{n}, t_{i+1}^{n}\right]$. So, we deduce that for $a=1, \ldots, d$ and any $\delta=2^{-n}$ for some integer $n$,

$$
\mathbb{E}\left[\left|\mathbf{B}_{t_{k}^{m}, t_{k+1}^{m}}^{a, 1, \delta}\right|^{q}\right] \leqslant C 2^{-m q / 2}
$$

Let $A_{s, t}^{a, b}\left(B^{\delta}(\omega)\right)$ be the area enclosed between the curve defined by $\left(B_{r}^{a, \delta}(\omega), B_{r}^{b, \delta}(\omega)\right)_{s \leqslant r \leqslant t}$ and its chord for $a, b \in\{1, \ldots, d\}$.

Let $m$ be an integer such that $m \leqslant n$, and let $k$ be an integer such that $k \in\left\{0, \ldots, 2^{m}-1\right\}$. Let $r$ belongs to $\left[t_{i}^{n}, t_{i+1}^{n}\right)$ with $t_{i}^{n} \geqslant t_{k}^{m}$. Then, it follows easily from (10.1) that

$$
\left|B_{r}^{a, \delta}-B_{t_{k}^{m}}^{a, \delta}\right| \leqslant\left|B_{t_{i+1}^{n}}^{a}-B_{t_{i}^{n}}^{a}\right|+\left|B_{t_{i}^{n}}^{a}-B_{t_{k}^{m}}^{a}\right|
$$

for $a=1, \ldots, d$, since $B_{t_{i}^{n}}^{\delta}=B_{t_{i}^{n}}$ for all $i \in\left\{0, \ldots, 2^{n}\right\}$. The Doob and the convexity inequalities imply that there exists a constant $C$ depending only on $q$ such that for $a=1, \ldots, d$,

$$
\begin{aligned}
\mathbb{E}\left[\sup _{r \in\left[t_{k}^{m}, t_{k+1}^{m}\right]}\left|B_{r}^{a, \delta}-B_{t_{k}^{m}}^{a, \delta}\right|^{q}\right] \leqslant & C 2^{q-1}\left(\mathbb{E}\left[\left|\left\langle B^{a}\right\rangle_{t_{i+1}^{n}}-\left\langle B^{a}\right\rangle_{t_{i}^{n}}\right|^{q / 2}\right]\right. \\
& \left.+\mathbb{E}\left[\left|\left\langle B^{a}\right\rangle_{t_{i}^{n}}-\left\langle B^{a}\right\rangle_{t_{k}^{m}}\right|^{q / 2}\right]\right) \\
\leqslant & C 2^{q-1}\left(2^{-n q / 2}+2^{-m q / 2}\right) \\
\leqslant & C 2^{q} 2^{-m q / 2}
\end{aligned}
$$


The last inequality holds since $m \leqslant n$.

Let $j$ and $j^{\prime}$ be such that $t_{j}^{n}=t_{k}^{m}$ and $t_{j^{\prime}}^{n}=t_{k}^{m}$. With the Doob inequality, there exists some constant $C^{\prime}$ such that, when one uses (11.3), for $a, b \in\{1, \ldots, d\}$,

$$
\begin{aligned}
\mathbb{E}\left[\left|\sum_{i=j}^{j^{\prime}-1}\left(B_{t_{i}^{n}}^{a, \delta}-B_{t_{k}^{m}}^{a, \delta}\right)\left(B_{t_{i+1}^{n}}^{b, \delta}-B_{t_{i}^{n}}^{b, \delta}\right)\right|^{q / 2}\right] \\
\leqslant C^{\prime} \mathbb{E}\left[\left(\sum_{i=j}^{j^{\prime}-1}\left(B_{t_{i}^{n}}^{a}-B_{t_{k}^{m}}^{a}\right)^{2}\left(\left\langle B^{b}\right\rangle_{t_{i+1}^{n}}-\left\langle B^{b}\right\rangle_{t_{i}^{n}}\right)\right)^{q / 4}\right] \\
\leqslant C^{\prime} \mathbb{E}\left[\sup _{r \in\left[t_{k}^{m}, t_{k+1}^{m}\right]}\left|B_{r}^{a}-B_{t_{k}^{m}}^{a}\right|^{q}\right]^{1 / 2}\left|t_{k+1}^{m}-t_{k}^{m}\right|^{q / 2} \\
\leqslant C^{\prime} \sqrt{C} 2^{q / 2} 2^{-m q / 4} .
\end{aligned}
$$

But it is easily verified that

$$
\begin{aligned}
A_{t_{k}^{m}, t_{k+1}^{m}}^{a, b}( & \left.B^{\delta}\right) \\
& =\frac{1}{4} \sum_{i=j}^{j^{\prime}-1}\left(B_{t_{i}^{n}}^{a}-B_{t_{k}^{m}}^{a}\right)\left(B_{t_{i+1}^{n}}^{b}-B_{t_{i}^{n}}^{b}\right)-\frac{1}{4} \sum_{i=j}^{j^{\prime}-1}\left(B_{t_{i}^{n}}^{b}-B_{t_{k}^{m}}^{b}\right)\left(B_{t_{i+1}^{n}}^{a}-B_{t_{i}^{n}}^{a}\right) .
\end{aligned}
$$

So, the inequality (11.4) implies that there exists some constant $C$ depending only on $q$ such that

$$
\mathbb{E}\left[\left|A_{t_{k}^{m}, t_{k+1}^{m}}^{a, b}\left(B^{\delta}\right)\right|^{q / 2}\right] \leqslant C 2^{-m q / 2} \text { for all } m \leqslant n .
$$

If $m \geqslant n$, the trajectory of $B^{\delta}$ between the times $t_{k}^{m}$ and $t_{k+1}^{m}$ is a straight line, so $A_{t_{k}^{m}, t_{k+1}^{m}}^{a, b}\left(B^{\delta}\right)=0$. So, (11.5) is true for all integers $n$ and $m$.

Since $A^{a, b}\left(B^{\delta}\right)$ is the antisymmetric part of $\mathbf{B}^{a, b, 2, \delta}$, the convexity inequality implies that for $a, b \in\{1, \ldots, d\}$

$$
\begin{aligned}
\left|\mathbf{B}_{t_{k}^{m}, t_{k+1}^{m}}^{a, b, 2, \delta}\right|^{q / 2} & \leqslant \frac{1}{2}\left|\mathbf{B}_{t_{k}^{m}, t_{k+1}^{m}}^{a, 1, \delta}\right| \cdot\left|\mathbf{B}_{t_{k}^{m}, t_{k+1}^{m}, 1, \delta}^{b, j}\right|+2^{q / 2-1}\left|A_{t_{k}^{m}, t_{k+1}^{m}}^{a, b}\left(B^{\delta}\right)\right|^{q / 2} \\
& \leqslant C 2^{-m q / 2}
\end{aligned}
$$

for some constant $C$ that depends only on $q$. For any $\beta>0$,

$$
\sum_{m \geqslant 1} m^{\beta} \sum_{i=0}^{2^{m}-1} 2^{-m q / 2}=\sum_{m \geqslant 1} m^{\beta} 2^{m(1-q / 2)}<+\infty
$$

since $1-q / 2<0$. So, one deduces from (11.2) that

$$
\sup _{\delta>0}\left(\mathbb{E}\left[\operatorname{Var}_{q}\left(\mathbf{B}^{1, \delta}\right)^{q}\right]+\mathbb{E}\left[\operatorname{Var}_{q / 2}\left(\mathbf{B}^{2, \delta}\right)^{q / 2}\right]\right)<+\infty .
$$


For $0 \leqslant u \leqslant v \leqslant 1$, let $m(v-u)$ is the smallest integer $m$ such that $\left[t_{k}^{m}, t_{k+1}^{m}\right] \subset[u, v]$ for some integer $k$. This quantity is deterministic and depends only on $u-v$. Owing to (11.1), for $\beta$ large enough and for all $\eta>0$,

$$
\sup _{\delta>0}\left(\mathbb{E}\left[\sup _{|t-s|<\eta}\left|\mathbf{B}_{s, t}^{1, \delta}\right|^{q}\right]+\mathbb{E}\left[\sup _{|t-s|<\eta}\left|\mathbf{B}_{s, t}^{2, \delta}\right|^{q / 2}\right]\right) \leqslant C \sum_{m \geqslant m(\eta)} m^{\beta} 2^{-m(1-q / 2)}
$$

for some constant $C$ that depends only on $q$ and $\beta$. Consequently, the series $\sum_{m \geqslant m(\eta)} m^{\beta} 2^{-m(1-q / 2)}$ may be arbitrary small if $\eta$ is chosen small enough.

Corollary 3 proves that $\left(\mathbf{B}^{\delta}\right)_{\delta>0}$ is tight in $\mathcal{G}^{p}\left(\mathbb{R}^{2}\right)$ for all $p>q>2$. But we already know from the Wong-Zakai theorem that $\mathbf{B}_{s, t}^{\delta}$ converges in probability to $\mathbf{B}_{s, t}$ uniformly in $(s, t) \in \Delta^{+}$. Thus, $\left(\mathbf{B}^{\delta}\right)_{\delta>0}$ converges in probability to $\mathbf{B}$ in $\mathcal{G}^{p}\left(\mathbb{R}^{d}\right)$.

\section{Applications to stochastic analysis}

The trajectories of stochastic processes are generally of finite $p$-variation with $p>2$. The typical case is of course that of the Brownian motion, whose trajectories are $\alpha$-Hölder continuous for all $\alpha<1 / 2$, and then of finite $p$-variation as soon as $p>2$. To apply the theory of rough paths to stochastic processes, the main difficulty is generally to construct the equivalent of the iterated integrals of the trajectories of the process.

The theory of rough paths has proved successful in many situations:

— Brownian motion and semi-martingales [32, 43].

- Reversible Markov Processes [1].

- Brownian motion on fractals [17].

- Fractional Brownian motion with Hurst exponent greater than 1/4 (note that if the Hurst exponent $h$ belongs to $(1 / 4,1 / 3]$, then third order iterated integrals have to be considered) [9, 10].

— Stochastic processes generated by divergence form operators [1, 27.

— Lévy processes [48, 49, 50].

— Gaussian processes in infinite dimension [24].

- Free Brownian Motion 3].

Further results or extensions of previous results to geometric multiplicative functionals also follow: 
- Flow of diffeomorphisms [32, 34].

- Calculus of variations [35].

- Large and small deviation principle [25, 42],

- Support theorems [25].

- Application to homogenization [28].

Some applications to numerical analysis are also provided:

- Pathwise construction of solutions of SDEs using path-dependant meshes [4, [15, 16].

— "Cubature formula" for computing weak solutions of SDEs numerically [37, 47].

\section{Bibliographical and historical remarks}

1. The article [32] is the synthesis of a series of works from T. Lyons and his co-authors: [30, 31, 33, 43,...

2. The idea of "pathwise" stochastic calculus is an old idea: see for example [12, 14]... But the theory of rough paths brings for the first time a theory of pathwise stochastic calculus valid for a large number of processes.

3. The use of the representation of the solution of some SDE using formally exponentials of iterated integrals have been also widely used: see for example the works [2, 11, 18, 21, 45] and related papers.

4. Stochastic Taylor expansions applied to numerical computations of solutions of SDEs has also given rise to an abundant literature: see for example [20] and references within. 5. (Related to Sect. 10.2). Shortly after being stated in [51, the theorem from E. Wong and M. Zakai on the approximations of SDEs by ordinary differential equations attracted many interest, and was extended in many directions. E. J. McShane was the first to show in [39] that different approximations of the trajectories may lead not to construct the Stratonovich integral, but the Stratonovich integral and a drift. An explicit construction is also given. There is now an important literature on such a corrective drift: See for example [8, 23, 46] and references within.

\section{References}

[1] Bass, R.F., Hambly, B., Lyons, T.J. (2002): Extending the Wong-Zakai theorem to reversible Markov processes. J. Eur. Math. Soc., 4:3, 237-269. $<$ DOI:10.1007/s100970200040>. [52, 57

[2] Ben Arous, G. (1989): Flots et séries de Taylor stochastiques. Probab. Theory Related Fields, 81:1, 29-77. 58 
[3] Capitaine, M., Donati-Martin, C. (2001): The Lévy area process for the free Brownian motion. J. Funct. Anal., 179:1, 153-169. <DOI:10.1006/jfan. $2000.3679>$. 52, 57

[4] Castell, F., Gaines, J. (1995): An efficient approximation method for stochastic differential equations by means of the exponential Lie series. Math. Comput. Simulation, 38:1-3, 13-19. Probabilités numériques (Paris, 1992). 58

[5] Chen, K.-T. (1957): Integration of paths, geometric invariants and a generalized Baker-Hausdorff formula. Ann. of Math. (2), 65, 163-178. 34

[6] Chen, K.-T. (1958): Integration of paths-a faithful representation, of paths by noncommutative formal power series. Trans. Amer. Math. Soc., 89:2, 395407. 4, 29

[7] Chistyakov, V.V., Galkin, O.E. (1998): On maps of bounded p-variations with $p>1$. Positivity, 2, 19-45. 15

[8] Cohen, S., Estrade, A. (2000): Non-symmetric approximations for manifoldvalued semimartingales. Ann. Inst. H. Poincaré, Probab. Stat., 36:1, 45-70. 58

[9] Coutin, L., Qian, Z. (2000): Stochastic differential equations for fractional Brownian motions. C. R. Acad. Sci. Paris Sér. I Math., 331:1, 75-80. 57

[10] Coutin, L., Qian, Z. (2002): Stochastic analysis, rough path analysis and fractional brownian motions. Probab. Theory Related Fields, 122:1, 108-140. $<$ DOI:0.1007/s004400100158>. 57

[11] Doss, H. (1977): Liens entre équations différentielles stochastiques et ordinaires. Ann. Inst. H. Poincaré Sect. B (N.S.), 13:2, 99-125. 58

[12] Dudley, R.M., Norvaiša, R. (1998): An introduction to $p$ variation and Young integrals - with emphasis on sample functions of stochastic processes. $<$ www.maphysto.dk/cgi-bin/w3-msql/publications/ genericpublication.html?publ=60>. Lecture given at the Centre for Mathematical Physics and Stochastics, Department of Mathematical Sciences, University of Aarhus. 3, 58

[13] Fliess, M., Normand-Cyrot, D. (1982): Algèbres de Lie nilpotentes, formule de Baker-Campbell-Hausdorff et intégrales itérées de K. T. Chen. In: Séminaire de Probabilités, XVI, pp 257-267. Springer, Berlin. 4 
[14] Föllmer, H. (1981): Calcul d'Itô sans probabilités. In: Séminaire de Probabilités, XV (Univ. Strasbourg, Strasbourg, 1979/1980), vol. 850 of Lecture Notes in Math., pp 143-150. Springer, Berlin. 58

[15] Gaines, J. G., Lyons, T. J. (1994): Random generation of stochastic area integrals. SIAM J. Appl. Math., 54:4, 1132-1146. 58

[16] Gaines, J. G., Lyons, T. J. (1997): Variable step size control in the numerical solution of stochastic differential equations. SIAM J. Appl. Math., 57:5, 14551484. 52, 58

[17] Hambly, B. M., Lyons, T. J. (1998): Stochastic area for Brownian motion on the Sierpinski gasket. Ann. Probab., 26:1, 132-148. 52, 57

[18] Hu, Y. Z. (1990): Calculs formels sur les EDS de Stratonovitch. In: Séminaire de Probabilités, XXIV, 1988/89, vol. 1426 of Lecture Notes in Math., pp 453460. Springer, Berlin. 58

[19] Ikeda, N., Watanabe, S. (1989): Stochastic Differential Equations and Diffusion Processes. North-Holland, 2nd ed. 7, 48, 51]

[20] Kloeden, P. E., Platen, E. (1992): Numerical solution of stochastic differential equations. Springer-Verlag, Berlin. 58

[21] Kunita, H. (1980): On the representation of solutions of stochastic differential equations. In: Séminaire de Probabilités, XIV (Paris, 1978/1979), vol. 784 of Lecture Notes in Math., pp 282-304. Springer, Berlin. 58

[22] Kunita, H. (1990): Stochastic flows and stochastic differential equations. Cambridge University Press. 48

[23] Kurtz, T. G., Protter, P. (1991): Wong-Zakai corrections, random evolutions, and simulation schemes for SDEs. In: Stochastic analysis, pp 331-346. Academic Press, Boston, MA. 58

[24] Ledoux, M., Lyons, T., Qian, Z. (2002): Lévy area of Wiener processes in Banach spaces. Ann. Probab., 30:2, 546-578. 7, 8, 48, 52, 55, 57

[25] Ledoux, M., Qian, Z., Zhang, T. (2002): Large deviations and support theorem for diffusions via rough paths. Stochastic Process. Appl., 102:2, 265-283. $<$ DOI:10.1016/S0304-4149(02)00176-X $>$. 58

[26] Lejay, A. (2002): On the convergence of stochastic integrals driven by processes converging on account of a homogenization property. Electron. J. Probab., 7:18, 1-18. <www.math.washington.edu/ ${ }^{\sim e j p e c p}>.49$ 
[27] Lejay, A. (2002): Stochastic differential equations driven by processes generated by divergence form operators. <www.iecn.u-nancy.fr/Preprint/ publis/lejay.Fri_Jun_21_17_03_23.html>. Institut Élie Cartan, Université Nancy I, preprint no. 23. 52, 55, 57]

[28] Lejay, A., Lyons, T.J. (2002): On the importance of the Lévy area for systems controlled by converging stochastic processes. Application to homogenization. In preparation. 49, 58

[29] Lévy, P. (1965): Processus stochastiques et mouvement brownien. GauthierVillars \& Cie, Paris, 2nd ed. 7

[30] Lyons, T. (1994): Differential equations driven by rough signals. I. An extension of an inequality of L. C. Young. Math. Res. Lett., 1:4, 451-464. 58

[31] Lyons, T. J. (1995): The interpretation and solution of ordinary differential equations driven by rough signals. In: Stochastic analysis (Ithaca, NY, 1993), pp 115-128. Amer. Math. Soc., Providence, RI. 58

[32] Lyons, T.J. (1998): Differential equations driven by rough signals. Rev. Mat. Iberoamericana, 14:2, 215-310. 2, 8, 21, 36, 40, 42, 44, 46, 47, 57, 58,

[33] Lyons, T. J., Qian, Z. M. (1996): Calculus for multiplicative functionals, Itô's formula and differential equations. In: Itô's stochastic calculus and probability theory, pp 233-250. Springer, Tokyo. 58

[34] Lyons, T. J., Qian, Z. (1997): Flow equations on spaces of rough paths. J. Funct. Anal., 149:1, 135-159. 58

[35] Lyons, T. J., Qian, Z. M. (1997): Calculus of variation for multiplicative functionals. In: New trends in stochastic analysis (Charingworth, 1994), pp 348-374. World Sci. Publishing, River Edge, NJ. 58

[36] Lyons, T., Qian, Z. (2002): System Control and Rough Paths. Oxford Mathematical Monographs. Oxford University Press. 2, 8

[37] Lyons, T., Victoir, N. (2002): Cubature on Wiener space. Oxford University (preprint). 7, 58

[38] Lyons, T., Zeitouni, O. (1999): Conditional exponential moments for iterated Wiener integrals. Ann. Probab., 27:4, 1738-1749. 52

[39] McShane, E. J. (1972): Stochastic differential equations and models of random processes. In: Proceedings of the Sixth Berkeley Symposium on Mathematical Statistics and Probability (Univ. California, Berkeley, Calif., 1970/1971), Vol. III: Probability theory, pp 263-294. Univ. California Press. 58 
[40] Reutenauer, C. (1993): Free Lie algebras, vol. 7 of London Mathematical Society Monographs. New Series. Oxford University Press. 31, 34

[41] Ryan, R.A. (2002): Introduction to Tensor Products of Banach Spaces. Springer-Verlag. 35

[42] Simon, T. (2002): Small deviations in $p$-variation norm for multidimensional Lévy processes. <www.maths.univ-evry.fr/pages_perso/simon/publi. html >. Université d'Evry (preprint). 58

[43] Sipiläinen, E.-M. (1993): A pathwise view of solutions of stochastic differential equations. PhD thesis, University of Edinburgh. 57, 58

[44] Stein, E. M. (1970): Singular integrals and differentiability properties of functions. Princeton University Press, Princeton, N.J. 44

[45] Sussmann, H.J. (1978): On the gap between deterministic and stochastic ordinary differential equations. Ann. Probability, 6:1, 19-41. 58

[46] Sussmann, H. J. (1991): Limits of the Wong-Zakai type with a modified drift term. In: Stochastic analysis, pp 475-493. Academic Press, Boston, MA. 58

[47] Victoir, N. (2002): Asymmetric cubature formulae with few points in high dimension for symmetric measures. Oxford University (preprint). 17, 34, 58

[48] Williams, D.R.E. (1998): Solutions of differential equations driven by cádlág paths of finite $p$-variations. $\mathrm{PhD}$ thesis, Imperial College, London. 57

[49] Williams, D.R.E. (2000): Diffeomorphic flows driven by Lévy processes. $<$ ArXivimath.PR/0001016>. Imperial College, London (preprint). 57

[50] Williams, D.R.E. (2002): Path-wise solutions of SDE's driven by Lévy processes. Rev. Mat. Iberoamericana, 17:2, 295-330. 57

[51] Wong, E., Zakai, M. (1965): On the convergence of ordinary integrals to stochastic integrals. Ann. Math. Statist., 36, 1560-1564. 48, 58

[52] Young, L.C. (1936): An inequality of the Hölder type, connected with Stieltjes integration. Acta Math., 67, 251-282. 3 